\title{
The [3 + 2] Annulation of Allylic Silanes in Acyclic Stereocontrol: Total Synthesis of (9S)-Dihydroerythronolide A
}

\author{
Zhi-Hui Peng and K. A. Woerpel* \\ Department of Chemistry, University of California \\ Irvine, CA 92697-2025
}

\section{Supporting Information}

\section{Contents:}

I. Ring-opening of the annulation product

II. Synthesis of the model aldol substrates and the model aldol reaction $\quad S-4$

III. Stereochemical proofs of the model aldol product

$S-12$

IV. Total synthesis of $(9 S)$-dihydroerythronolide A

$S-15$

V. Analytical Data

$S-30$

General. ${ }^{1} \mathrm{H}$ NMR and ${ }^{13} \mathrm{C}$ NMR spectra were recorded at ambient temperature at $500 \mathrm{MHz}$ and $125 \mathrm{MHz}$, respectively, using a Bruker DRX 500 spectrometer. The data are reported as follows: chemical shift in ppm from internal tetramethylsilane on the $\delta$ scale, multiplicity $(\mathrm{br}=$ broad, $\mathrm{s}=$ singlet, $\mathrm{d}=$ doublet, $\mathrm{t}=$ triplet, $\mathrm{q}=$ quartet, $\mathrm{m}=$ multiplet), coupling constants $(\mathrm{Hz})$ and integration. High resolution mass spectra were acquired on a VG Analytical 7070E or Fisons Autospec spectrometer, and were obtained by peak matching. Microanalyses were performed by Atlantic Microlab, Atlanta, GA. Melting points are reported uncorrected. Analytical thin layer chromatography was performed using EM Reagents $0.25 \mathrm{~mm}$ silica gel 60-F plates. Liquid chromatography was performed using forced flow (flash chromatography) of the indicated solvent system on EM Reagents silica gel $\left(\mathrm{SiO}_{2}\right) 60$ (230-400 mesh). Enantiomeric excesses were determined by HPLC analysis on a Hewlett Packard series 1100 using a Chiralcel OJ column, and the enantiomerically enriched material was compared with racemic material. All reactions were carried out under an atmosphere of nitrogen in glassware which had been flame-dried under a stream of nitrogen. Unless otherwise noted, all reagents were commercially obtained and, where appropriate, purified prior to use. THF, $\mathrm{Et}_{2} \mathrm{O}$, and $\mathrm{CH}_{2} \mathrm{Cl}_{2}$ were dried by filtration through alumina according to the procedure of Grubbs. ${ }^{1}$

\section{Ring-opening of the annulation product}<smiles>CCC=C(C)C=O</smiles>

19

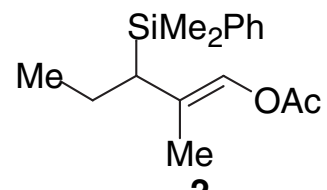

2

Acetic acid 2-methyl-3-(dimethylphenylsilyl)-(E)-pent-1-enyl ester (2). To a solution of $\mathrm{PhMe}_{2} \mathrm{SiCl}(16.8$ $\mathrm{mL}, 100 \mathrm{mmol})$ in $100 \mathrm{~mL}$ of THF was added lithium wire $(4.14 \mathrm{~g}, 600 \mathrm{mmol})$. After $16 \mathrm{~h}$ at $23{ }^{\circ} \mathrm{C}$, the resultant dark red solution was added to a cooled $\left(-30^{\circ} \mathrm{C}\right)$ suspension of $\mathrm{CuI}(9.50 \mathrm{~g}, 50.0 \mathrm{mmol})$ in $10 \mathrm{~mL}$ of THF by cannula. After $4 \mathrm{~h}$ at $-30{ }^{\circ} \mathrm{C}$, the slurry was cooled to $-78{ }^{\circ} \mathrm{C}$, followed by the addition of aldehyde 19 $(5.70 \mathrm{~mL}, 50.0 \mathrm{mmol})$ dropwise by syringe. The reaction mixture was stirred at $-78{ }^{\circ} \mathrm{C}$ for $1 \mathrm{~h}$ and was warmed to $-30{ }^{\circ} \mathrm{C}$ for an additional $2 \mathrm{~h}$. $\mathrm{Ac}_{2} \mathrm{O}(18.9 \mathrm{~mL}, 200 \mathrm{mmol})$ was then added and the reaction mixture was

\footnotetext{
${ }^{1}$ Pangborn, A. B.; Giardello, M. A.; Grubbs, R. H.; Rosen, R. K.; Timmers, F. J. Organometallics 1996, 15, $1518-1520$.
} 
allowed to warm to $23{ }^{\circ} \mathrm{C}$, and after $12 \mathrm{~h}, 200 \mathrm{~mL}$ of $\mathrm{Et}_{2} \mathrm{O}$ was added. The mixture was washed sequentially with $100 \mathrm{~mL}$ of $1 \mathrm{~N} \mathrm{HCl}, 50 \mathrm{~mL}$ of saturated aqueous $\mathrm{NaHCO}_{3}$ solution, and $30 \mathrm{~mL}$ of brine. The organic layer was dried $\left(\mathrm{MgSO}_{4}\right)$, filtered, and concentrated in vacuo. Purification by flash chromatography (benzene) afforded 2 as a colorless oil $(13.5 \mathrm{~g}, 98 \%):{ }^{1} \mathrm{H} \mathrm{NMR}\left(\mathrm{CDCl}_{3}, 500 \mathrm{MHz}\right) \delta 7.54(\mathrm{~m}, 2 \mathrm{H}), 7.38(\mathrm{~m}, 3 \mathrm{H}), 6.86(\mathrm{~d}, J$ $=1.4 \mathrm{~Hz}, 1 \mathrm{H}), 2.14(\mathrm{~s}, 3 \mathrm{H}), 1.60-1.49(\mathrm{~m}, 3 \mathrm{H}), 1.54(\mathrm{~d}, J=1.4 \mathrm{~Hz}, 3 \mathrm{H}), 0.86(\mathrm{~d}, J=7.2 \mathrm{~Hz}, 3 \mathrm{H}), 0.36(\mathrm{~s}, 3 \mathrm{H})$, $0.31(\mathrm{~s}, 3 \mathrm{H}) ;{ }^{13} \mathrm{C} \mathrm{NMR}\left(\mathrm{CDCl}_{3}, 125 \mathrm{MHz}\right) \delta 168.0,138.1,133.8,130.0,128.9,127.6,122.1,35.3,20.7,20.5$, 13.9, 13.3, -3.6, -4.4; IR (thin film) 1749, $1227 \mathrm{~cm}^{-1}$; HRMS $\left(\mathrm{CI} / \mathrm{NH}_{3}\right) \mathrm{m} / z$ calcd for $\mathrm{C}_{16} \mathrm{H}_{24} \mathrm{O}_{2} \mathrm{Si}\left(\mathrm{M}^{+}\right)$ 276.1545, found 276.1541. Anal. Calcd for $\mathrm{C}_{16} \mathrm{H}_{24} \mathrm{O}_{2} \mathrm{Si}$ : C, 69.53; H, 8.76. Found: C, 69.26; H, 8.85.
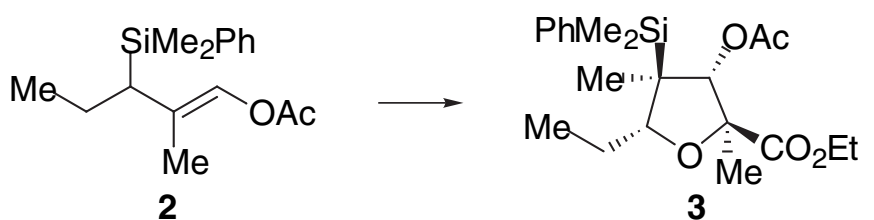

$\left(2 S^{*}, 3 R^{*}, 4 R^{*}, 5 R^{*}\right)$-3-Acetoxy-4-dimethylphenylsilyl-2,4-dimethyl-5-ethyltetrahydrofuran-2-carboxylic acid ethyl ester (3). To a cooled $\left(-78{ }^{\circ} \mathrm{C}\right)$ solution of ethyl pyruvate $(1.83 \mathrm{~mL}, 5.00 \mathrm{mmol})$ and allylsilane 2 $(4.14 \mathrm{~g}, 15.0 \mathrm{mmol})$ in $75 \mathrm{~mL}$ of dry $\mathrm{CH}_{2} \mathrm{Cl}_{2}$ was added dropwise $\mathrm{SnCl}_{4}\left(0.200 \mathrm{M}\right.$ solution in $\mathrm{CH}_{2} \mathrm{Cl}_{2}, 82.5 \mathrm{~mL}$, $16.5 \mathrm{mmol}$ ) by syringe. After $1 \mathrm{~h}, 3.0 \mathrm{~mL}$ of $\mathrm{Et}_{3} \mathrm{~N}$ was added followed by $40 \mathrm{~mL}$ of water. The mixture was warmed to $23{ }^{\circ} \mathrm{C}$ and the layers were separated. The aqueous layer was extracted with $3 \times 60 \mathrm{~mL}$ of EtOAc. The combined organic layers were washed with $10 \mathrm{~mL}$ of brine, dried $\left(\mathrm{MgSO}_{4}\right)$, filtered, and concentrated in vacuo. Purification by flash chromatography (5:95 to 10:90 EtOAc/hexanes) afforded a colorless oil $\mathbf{3}(4.50 \mathrm{~g}$, $77 \%)$ as a single stereoisomer as determined by ${ }^{1} \mathrm{H}$ NMR spectroscopy of the crude product: ${ }^{1} \mathrm{H}$ NMR $\left(\mathrm{CDCl}_{3}\right.$, $500 \mathrm{MHz}) \delta 7.50(\mathrm{~m}, 2 \mathrm{H}), 7.39(\mathrm{~m}, 3 \mathrm{H}), 5.62(\mathrm{~s}, 1 \mathrm{H}), 4.15(\mathrm{~m}, 2 \mathrm{H}), 3.92(\mathrm{dd}, J=10.2,1.7 \mathrm{~Hz}, 1 \mathrm{H}), 1.85(\mathrm{~s}$, $3 \mathrm{H}), 1.48(\mathrm{~m}, 1 \mathrm{H}), 1.33(\mathrm{~m}, 1 \mathrm{H}), 1.26(\mathrm{~s}, 3 \mathrm{H}), 1.23(\mathrm{t}, J=7.1 \mathrm{~Hz}, 3 \mathrm{H}), 1.06(\mathrm{~s}, 3 \mathrm{H}), 1.01(\mathrm{t}, J=7.4 \mathrm{~Hz}, 3 \mathrm{H})$, 0.38 (s, 3H), 0.37 (s, 3H); ${ }^{13} \mathrm{C}$ NMR $\left(\mathrm{CDCl}_{3}, 125 \mathrm{MHz}\right) \delta 173.7,169.8,135.8,134.2,129.3,127.7,84.1,82.0$, 79.7, 60.9, 35.4, 23.7, 20.3 19.8, 14.0, 11.8, 10.1, -5.5, -5.7; IR (thin film) $1747,1731 \mathrm{~cm}^{-1}$; HRMS (CI/ $\left.\mathrm{NH}_{3}\right)$ $m / z$ calcd for $\mathrm{C}_{20} \mathrm{H}_{29} \mathrm{O}_{5} \mathrm{Si},\left(\mathrm{M}-\mathrm{CH}_{3}\right)^{+}$377.1784, found 377.1786. Anal. Calcd for $\mathrm{C}_{21} \mathrm{H}_{32} \mathrm{O}_{5} \mathrm{Si}: \mathrm{C}, 64.25 ; \mathrm{H}$, 8.22. Found: $\mathrm{C}, 64.47 ; \mathrm{H}, 8.24$.
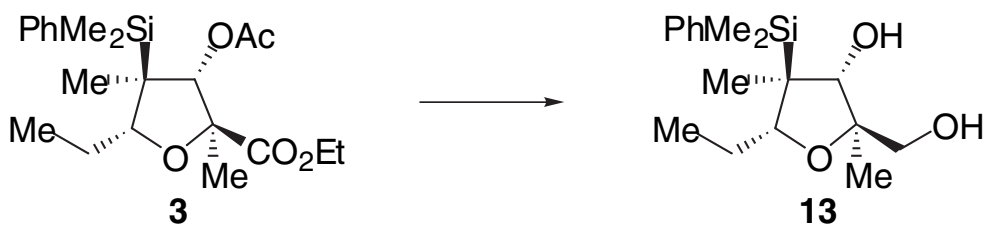

$\left(2 R^{*}, 3 R^{*}, 4 R^{*}, 5 R^{*}\right)$-4-Dimethylphenylsilyl-3-hydroxy-2-hydroxymethyl-2,4-dimethyl-5-

ethyltetrahydrofuran (13). To a cooled $\left(0^{\circ} \mathrm{C}\right)$ suspension of $\mathrm{LiAlH}_{4}(0.736 \mathrm{~g}, 19.4 \mathrm{mmol})$ in $40 \mathrm{~mL}$ of $\mathrm{Et}_{2} \mathrm{O}$ was added a solution of $3(3.80 \mathrm{~g}, 9.70 \mathrm{mmol})$ in $40 \mathrm{~mL}$ of $\mathrm{Et}_{2} \mathrm{O}$. After $10 \mathrm{~h}$ at $23{ }^{\circ} \mathrm{C}$, the excess hydride was carefully quenched by $3 \mathrm{~g}$ of $\mathrm{Na}_{2} \mathrm{SO}_{4} \cdot 10 \mathrm{H}_{2} \mathrm{O}$. The mixture was filtered and the solid was washed with $3 \times 50$ $\mathrm{mL}$ of EtOAc. The combined filtrate was concentrated in vacuo. Purification by flash chromatography (30:70 to 50:50 EtOAc/hexanes) afforded $\mathbf{1 3}$ as a white solid $(2.92 \mathrm{~g}, 98 \%)$ : $\mathrm{mp} 91-93{ }^{\circ} \mathrm{C} ;{ }^{1} \mathrm{H} \mathrm{NMR}\left(\mathrm{CDCl}_{3}, 500\right.$ MHz) $\delta 7.53(\mathrm{~m}, 2 \mathrm{H}), 7.40(\mathrm{~m}, 3 \mathrm{H}), 4.10(\mathrm{~d}, J=6.2 \mathrm{~Hz}, 1 \mathrm{H}), 3.56(\mathrm{dd}, J=10.1,1.8 \mathrm{~Hz}, 1 \mathrm{H}), 3.33(\mathrm{dd}, J=11.0$, $5.5 \mathrm{~Hz}, 1 \mathrm{H}), 3.29(\mathrm{dd}, J=11.0,7.4 \mathrm{~Hz}, 1 \mathrm{H}), 1.73(\mathrm{dd}, J=7.3,5.5 \mathrm{~Hz}, 1 \mathrm{H}), 1.57(\mathrm{~d}, J=6.2 \mathrm{~Hz}, 1 \mathrm{H}), 1.47(\mathrm{~m}$, $1 \mathrm{H}), 1.33(\mathrm{~m}, 1 \mathrm{H}), 1.13(\mathrm{~s}, 3 \mathrm{H}), 1.00(\mathrm{~s}, 3 \mathrm{H}), 0.98(\mathrm{t}, J=7.4 \mathrm{~Hz}, 3 \mathrm{H}), 0.38(\mathrm{~s}, 3 \mathrm{H}), 0.37(\mathrm{~s}, 3 \mathrm{H}),{ }^{13} \mathrm{C} \mathrm{NMR}$ 
$\left(\mathrm{CDCl}_{3}, 125 \mathrm{MHz}\right) \delta 136.3,134.0,129.5,128.1,82.3,81.7,78.0,68.1,37.0,24.3,19.6,12.1,9.1,-5.3,-5.5 ; \mathrm{IR}$ (KBr) $3426 \mathrm{~cm}^{-1}$; HRMS $\left(\mathrm{CI} / \mathrm{NH}_{3}\right) \mathrm{m} / z$ calcd for $\mathrm{C}_{16} \mathrm{H}_{25} \mathrm{O}_{2} \mathrm{Si}\left(\mathrm{M}-\mathrm{CH}_{2} \mathrm{OH}\right)^{+}$277.1624, found 277.1629. Anal. Calcd for $\mathrm{C}_{17} \mathrm{H}_{28} \mathrm{O}_{3} \mathrm{Si}$ : C, 66.19; H, 9.16. Found: C, 66.16; H, 9.19.

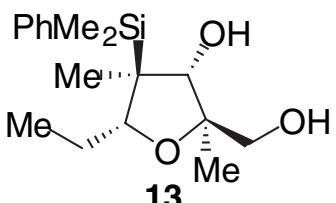

13

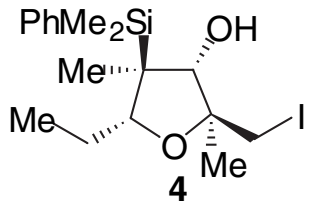

$\left(2 S^{*}, 3 R^{*}, 4 R^{*}, 5 R^{*}\right)$-2,4-Dimethyl-4-dimethylphenylsilyl-5-ethyl-3-hydroxy-2-iodomethyltetrahydrofuran

(4). To a solution of $13(0.466 \mathrm{~g}, 1.51 \mathrm{mmol})$ in $20 \mathrm{~mL}$ of benzene was added $\mathrm{Ph}_{3} \mathrm{P}(0.476 \mathrm{~g}, 1.82 \mathrm{mmol})$, imidazole $(0.248 \mathrm{~g}, 3.64 \mathrm{mmol})$, and $\mathrm{I}_{2}(0.462 \mathrm{~g}, 1.82 \mathrm{mmol})$. The mixture was heated at reflux for $1 \mathrm{~h}$. After being cooled to $23{ }^{\circ} \mathrm{C}$, the mixture was filtered through Celite, and the cake was washed with $3 \times 20 \mathrm{~mL}$ of ether. The filtrate was concentrated in vacuo. Purification by flash chromatography (2:98 to 5:95 EtOAc/hexanes) afforded 4 as a colorless oil $(0.500 \mathrm{~g}, 79 \%):{ }^{1} \mathrm{H}$ NMR $\left(\mathrm{CDCl}_{3}, 500 \mathrm{MHz}\right) \delta 7.53(\mathrm{~m}, 2 \mathrm{H}), 7.41$ $(\mathrm{m}, 3 \mathrm{H}), 4.18(\mathrm{~d}, J=5.7 \mathrm{~Hz}, 1 \mathrm{H}), 3.56(\mathrm{dd}, J=9.9,1.2 \mathrm{~Hz}, 1 \mathrm{H}), 3.09(\mathrm{~d}, J=9.7 \mathrm{~Hz}, 1 \mathrm{H}), 3.01(\mathrm{~d}, J=9.7 \mathrm{~Hz}$, $1 \mathrm{H}), 1.44(\mathrm{~m}, 1 \mathrm{H}), 1.32(\mathrm{~s}, 3 \mathrm{H}$ and $\mathrm{m}, 1 \mathrm{H}), 1.25(\mathrm{~d}, J=5.7 \mathrm{~Hz}, 1 \mathrm{H}), 1.00(\mathrm{~s}, 3 \mathrm{H}), 0.96(\mathrm{t}, J=7.4 \mathrm{~Hz}, 3 \mathrm{H}), 0.41$ $(\mathrm{s}, 3 \mathrm{H}), 0.40(\mathrm{~s}, 3 \mathrm{H}) ;{ }^{13} \mathrm{C} \mathrm{NMR}\left(\mathrm{CDCl}_{3}, 125 \mathrm{MHz}\right) \delta 136.2,134.1,129.6,128.1,81.8,81.0,79.8,38.1,23.9$, 22.5, 17.8, 12.0, 8.5, -5.3; IR (thin film) $3578,1252,1110 \mathrm{~cm}^{-1}$; HRMS $\left(\mathrm{CI} / \mathrm{NH}_{3}\right) \mathrm{m} / z$ calcd for $\mathrm{C}_{17} \mathrm{H}_{27} \mathrm{IO}_{2} \mathrm{Si}$ $\left(\mathrm{M}^{+}\right)$418.0825, found 418.0826. Anal. Calcd for $\mathrm{C}_{17} \mathrm{H}_{27} \mathrm{IO}_{2} \mathrm{Si}$ : C, 48.80; H, 6.50. Found: $\mathrm{C}, 49.11 ; \mathrm{H}, 6.65$.

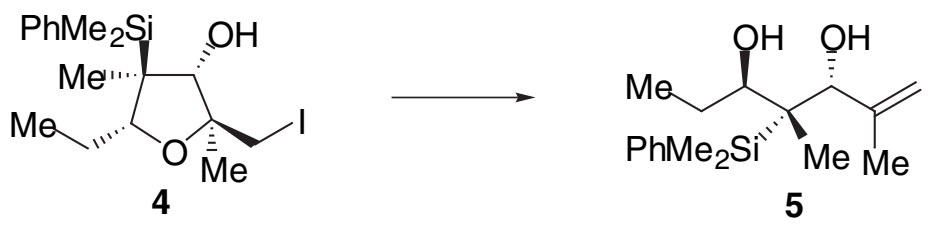

$\left(3 R^{*}, 4 R^{*}, 5 R^{*}\right)$-2,4-Dimethyl-4-dimethylphenylsilylhept-1-en-3,5-diol (5). To a solution of the iodide 4 $(0.320 \mathrm{~g}, 0.770 \mathrm{mmol})$ in $3 \mathrm{~mL}$ of $\mathrm{Et}_{2} \mathrm{O}$ and $3 \mathrm{~mL}$ of $\mathrm{MeOH}$ was added zinc powder $(0.504 \mathrm{~g}, 7.70 \mathrm{mmol})$ followed by $\mathrm{AcOH}(0.095 \mathrm{~mL}, 1.54 \mathrm{mmol})$. The reaction mixture was stirred for $12 \mathrm{~h}$ at $23{ }^{\circ} \mathrm{C}$, at which point the reaction mixture was diluted with $50 \mathrm{~mL}$ of $\mathrm{Et}_{2} \mathrm{O}$ and filtered. The filtrate was washed with $20 \mathrm{~mL}$ of saturated aqueous $\mathrm{NaHCO}_{3}$, brine $(10 \mathrm{~mL})$, dried $\left(\mathrm{MgSO}_{4}\right)$, and concentrated in vacuo. Purification by flash chromatography (5:95 to $10: 90 \mathrm{EtOAc} /$ hexanes) afforded 5 as a white solid $(0.183 \mathrm{~g}, 82 \%): \mathrm{mp} 120-122{ }^{\circ} \mathrm{C} ;{ }^{1} \mathrm{H}$ NMR (CDCl, $500 \mathrm{MHz}) \delta 7.73(\mathrm{~m}, 2 \mathrm{H}), 7.39(\mathrm{~m}, 3 \mathrm{H}), 5.28(\mathrm{~s}, 1 \mathrm{H}), 5.08(\mathrm{~s}, 1 \mathrm{H}), 4.35(\mathrm{~s}, 1 \mathrm{H}), 3.32(\mathrm{~d}, J=8.2$ $\mathrm{Hz}, 1 \mathrm{H}), 3.21(\mathrm{t}, J=8.5 \mathrm{~Hz}, 1 \mathrm{H}), 2.96(\mathrm{br}, 1 \mathrm{H}), 1.81(\mathrm{~s}, 3 \mathrm{H}), 1.67(\mathrm{~m}, 1 \mathrm{H}), 1.57(\mathrm{~m}, 1 \mathrm{H}), 1.05(\mathrm{~s}, 3 \mathrm{H}), 1.01(\mathrm{t}, J$ $=7.3 \mathrm{~Hz}, 3 \mathrm{H}), 0.63(\mathrm{~s}, 3 \mathrm{H}), 0.47(\mathrm{~s}, 3 \mathrm{H}) ;{ }^{13} \mathrm{C} \mathrm{NMR}\left(\mathrm{CDCl}_{3}, 125 \mathrm{MHz}\right) \delta 147.7,139.0,135.2,128.5,127.3$, 114.0, 81.8, 81.3, 37.6, 23.6, 21.9, 18.3, 11.4, -0.6, -1.5; IR (thin film) 3346, 1428, $1099 \mathrm{~cm}^{-1}$; HRMS (CI/ $\left.\mathrm{NH}_{3}\right)$ $m / z$ calcd for $\mathrm{C}_{17} \mathrm{H}_{26} \mathrm{OSi}\left(\mathrm{M}-\mathrm{H}_{2} \mathrm{O}\right)^{+} 274.1753$, found 274.1755. Anal. Calcd for $\mathrm{C}_{17} \mathrm{H}_{28} \mathrm{O}_{2} \mathrm{Si}$ : C, 69.81; H, 9.65. Found: C, 69.73; H, 9.39. 


\section{Synthesis of the model aldol substrates and the model aldol reaction}

\section{Synthetic scheme:}

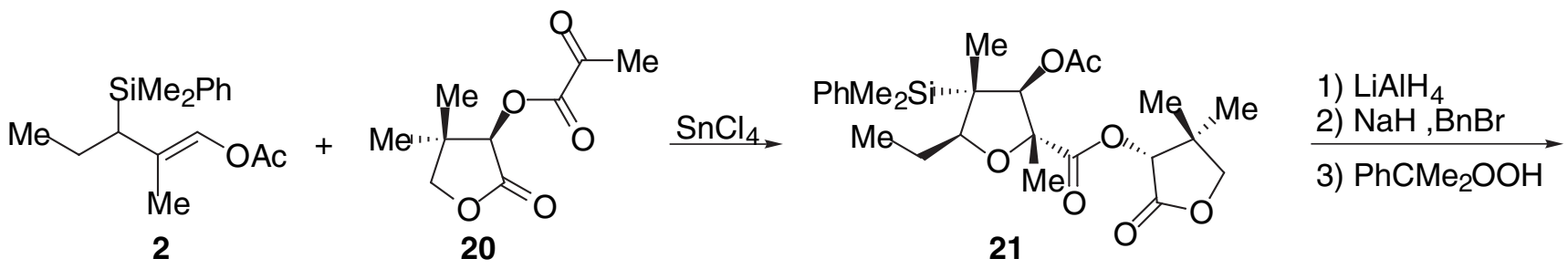

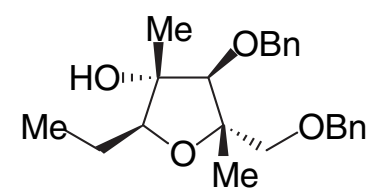

22

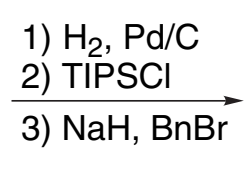

2) $\mathrm{TIPSCl}$

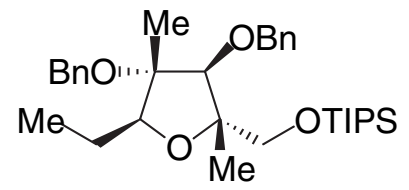

23
1) TBAF
2) $I_{2}$
3) $\mathrm{Zn}$
4) Swern

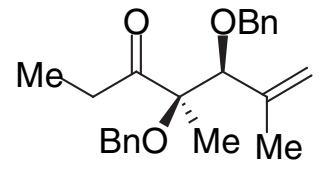

10

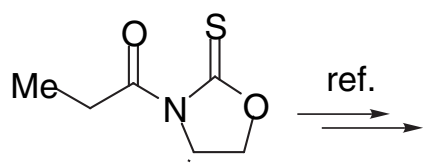

B⿳亠े<smiles>CC(C)C(O)C(C)CO</smiles>

25
1) $\mathrm{ArCH}(\mathrm{OMe})_{2}$

2) $i-\mathrm{Bu}_{2} \mathrm{AlH}$

3) Swern

24<smiles>[H][R16]([H])(C)OC(C(C)C)C(C=O)C(C)C</smiles>

11<smiles>CCC(SCCc1ccccc1)C(C)=COC(C)=O</smiles>

$( \pm)-2$

(+)-20

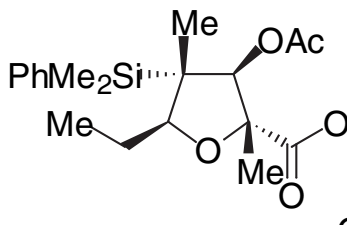

$(-)-21$

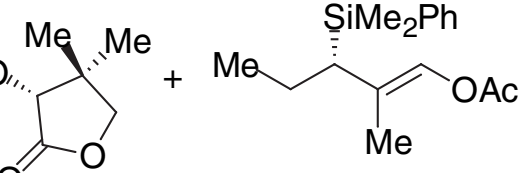

$(-)-2$

$(2 R, 3 S, 4 S, 5 S)$-3-Acetoxy-4-dimethylphenylsilyl-2,4-dimethyl-5-ethyl-tetrahydrofuran-2-carboxylic acid

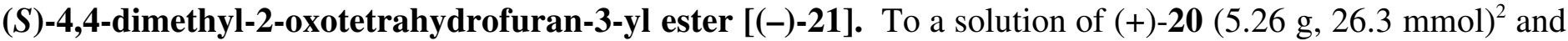
$( \pm)$-allylsilane $2(13.8 \mathrm{~g}, 50.0 \mathrm{mmol})$ in $120 \mathrm{~mL}$ of dry $\mathrm{CH}_{2} \mathrm{Cl}_{2}$ was added dropwise $\mathrm{SnCl}_{4}(0.200 \mathrm{M}$ solution in $\mathrm{CH}_{2} \mathrm{Cl}_{2}, 137 \mathrm{~mL}, 27.4 \mathrm{mmol}$ ) by syringe at $-78^{\circ} \mathrm{C}$. After $24 \mathrm{~h}$ at $-78{ }^{\circ} \mathrm{C}, 10 \mathrm{~mL}$ of $\mathrm{Et}_{3} \mathrm{~N}$ was added followed by $200 \mathrm{~mL}$ of water. The layers were separated and the aqueous layer was extracted with $3 \times 200 \mathrm{~mL}$ of EtOAc. The combined organic layers were washed with $50 \mathrm{~mL}$ of brine, dried $\left(\mathrm{MgSO}_{4}\right)$, filtered, and concentrated in vacuo. Purification by flash chromatography (5:95 to 30:70 EtOAc/hexanes) afforded (-)-2 (6.81 g, 49\%) as a colorless oil and (-)-21 $(9.53 \mathrm{~g}, 80 \%)$ as a white solid. The spectra data of the resolved (-)-2 were identical to the racemic one (vide supra): $[\alpha]_{\mathrm{D}}^{23}-18.6\left(\right.$ c 1.02, $\left.\mathrm{CHCl}_{3}\right)$. Compound (-)-21 was determined to be a single stereoisomer by ${ }^{1} \mathrm{H}$ NMR spectroscopy analysis of the crude mixture: $\mathrm{mp} 153-155{ }^{\circ} \mathrm{C} ;{ }^{1} \mathrm{H}$ NMR $\left(\mathrm{CDCl}_{3}, 500\right.$ MHz) $\delta 7.44(\mathrm{~m}, 2 \mathrm{H}), 7.33(\mathrm{~m}, 3 \mathrm{H}), 5.66(\mathrm{~s}, 1 \mathrm{H}), 5.32(\mathrm{~s}, 1 \mathrm{H}), 4.02(\mathrm{~m}, 3 \mathrm{H}), 1.77(\mathrm{~s}, 3 \mathrm{H}), 1.48(\mathrm{~m}, 1 \mathrm{H}), 1.33$

\footnotetext{
${ }^{2}$ Peng, Z.-H.; Woerpel, K. A. Org. Lett. 2002, 4, 2945-2948.
} 
$(\mathrm{m}, 1 \mathrm{H}), 1.28(\mathrm{~s}, 3 \mathrm{H}), 1.23(\mathrm{~s}, 3 \mathrm{H}), 1.11(\mathrm{~s}, 3 \mathrm{H}), 1.07(\mathrm{~s}, 3 \mathrm{H}), 1.02(\mathrm{t}, J=7.3 \mathrm{~Hz}, 3 \mathrm{H}), 0.36(\mathrm{~s}, 3 \mathrm{H}), 0.35$ (s, 3H); ${ }^{13} \mathrm{C}$ NMR $\left(\mathrm{CDCl}_{3}, 125 \mathrm{MHz}\right) \delta 172.2,171.7,170.1,135.8,134.1,129.3,127.7,84.1,81.9,79.4,76.0,75.7$, 40.6, 35.5, 23.5, 23.0, 20.1, 19.8, 19.7, 11.7, 10.0, -5.4, -5.6; IR (KBr) 1794, 1752; $[\alpha]^{23}{ }_{\mathrm{D}}-72.1$ (c 1.20, $\mathrm{CHCl}_{3}$ ); HRMS $\left(\mathrm{CI} / \mathrm{NH}_{3}\right) \mathrm{m} / \mathrm{z}$ calcd for $\mathrm{C}_{25} \mathrm{H}_{36} \mathrm{O}_{7} \mathrm{Si}\left(\mathrm{M}^{+}\right)$476.2230, found 476.2229. Anal. Calcd for $\mathrm{C}_{25} \mathrm{H}_{36} \mathrm{O}_{7} \mathrm{Si}: \mathrm{C}, 63.00 ; \mathrm{H}, 7.61$. Found: $\mathrm{C}, 62.88 ; \mathrm{H}, 7.65$.

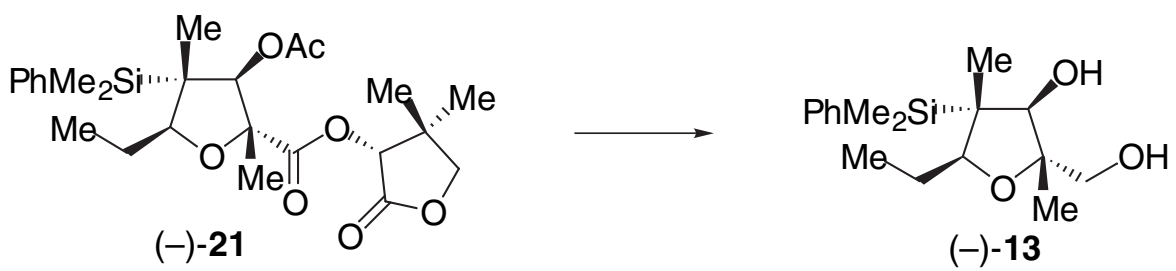

(2S,3S,4S,5S)-4-Dimethylphenylsilyl-3-hydroxy-2-hydroxymethyl-2,4-dimethyl-5-ethyltetrahydrofuran [(-)-13]. To a cooled $\left(0{ }^{\circ} \mathrm{C}\right)$ suspension of $\mathrm{LiAlH}_{4}(2.90 \mathrm{~g}, 76.4 \mathrm{mmol})$ in $100 \mathrm{~mL}$ of THF was added a solution of $(-)-21(9.10 \mathrm{~g}, 19.1 \mathrm{mmol})$ in $100 \mathrm{~mL}$ of THF. After $12 \mathrm{~h}$, the excess hydride was carefully quenched by addition of $10 \mathrm{~g}$ of $\mathrm{Na}_{2} \mathrm{SO}_{4} \cdot 10 \mathrm{H}_{2} \mathrm{O}$. The mixture was filtered and the solid was washed with $3 \times 100 \mathrm{~mL}$ of EtOAc. The combined filtrate was concentrated in vacuo. Purification by flash chromatography (30:70 to 50:50 EtOAc/hexanes) afforded (-)-13 as a white solid (5.45 g, 93\%) with an ee of $>98 \%$ as determined by chiral HPLC analysis: $[\alpha]^{23}{ }_{\mathrm{D}}-46.9\left(c 1.40, \mathrm{CHCl}_{3}\right) ; \mathrm{mp} 93-94{ }^{\circ} \mathrm{C}$; The spectral data of $(-)-\mathbf{1 3}$ were identical to the racemic compound (vide supra).

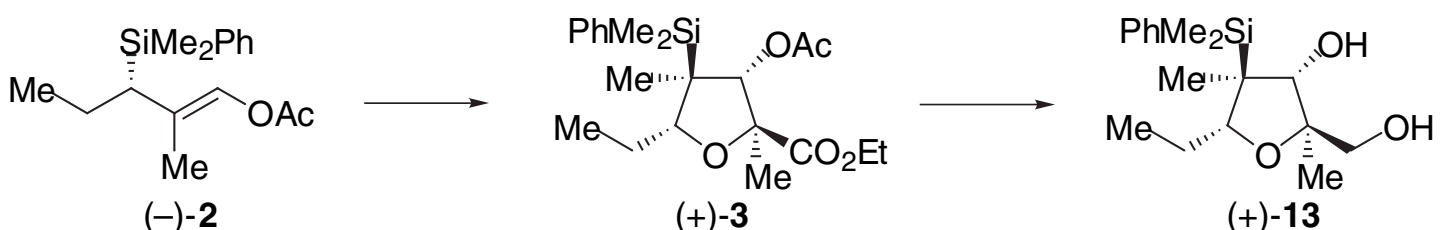

$(2 S, 3 R, 4 R, 5 R)-3$-Acetoxy-4-dimethylphenylsilyl-2,4-dimethyl-5-ethyl-tetrahydrofuran-2-carboxylic acid ethyl ester $[(+)-3]$ and $(2 R, 3 R, 4 R, 5 R)$-4-Dimethylphenylsilyl-3-hydroxy-2-hydroxymethyl-2,4-dimethyl-5ethyltetrahydrofuran [(+)-13]. The same procedures developed for the racemic compounds were employed. Diol (+)-13 was obtained as a white solid with an ee of $>99 \%$ as determined by chiral HPLC analysis. The spectral data of $(+)-\mathbf{3}$ and $(+)-\mathbf{1 3}$ were identical to the racemic ones (vide supra): $(+)-\mathbf{3}:[\alpha]_{\mathrm{D}}^{23}+38.5(c 1.38$, $\left.\mathrm{CHCl}_{3}\right) ;(+)-\mathbf{1 3}:[\alpha]_{\mathrm{D}}^{23}+47.7\left(\right.$ c 1.20, $\left.\mathrm{CHCl}_{3}\right)$.

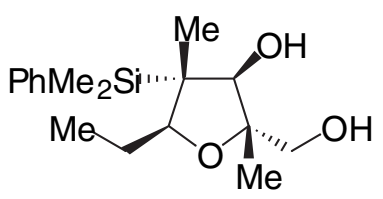

$(-)-13$

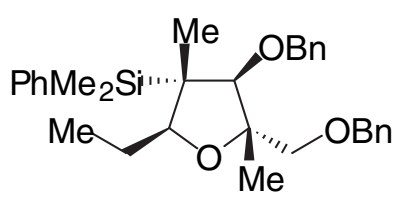

26

(2S,3S,4S,5S)-3-Benzyloxy-2-benzyloxymethyl-2,4-dimethyl-4-dimethylphenylsilyl-5-

ethyltetrahydrofuran (26). To a suspension of $\mathrm{NaH}(4.10 \mathrm{~g}, 60 \%$ oil dispersion, $102.6 \mathrm{mmol}$, washed with $3 \times$ $40 \mathrm{~mL}$ of hexanes) in $70 \mathrm{~mL}$ of DMF was added a solution of diol (-)-13 (7.90 g, $25.6 \mathrm{mmol})$ in $70 \mathrm{~mL}$ of DMF at $0{ }^{\circ} \mathrm{C}$. After $0.5 \mathrm{~h}, \mathrm{BnBr}(12.2 \mathrm{~mL}, 102.6 \mathrm{mmol})$ and $n$ - $\mathrm{Bu}_{4} \mathrm{NI}(0.472 \mathrm{~g}, 1.28 \mathrm{mmol})$ were added at $0^{\circ} \mathrm{C}$, and the reaction mixture was allowed to warm to $23{ }^{\circ} \mathrm{C}$. After $12 \mathrm{~h}$, a solution of saturated aqueous $\mathrm{NH}_{4} \mathrm{Cl}(100$ $\mathrm{mL}$ ) was added. The mixture was extracted with $3 \times 200 \mathrm{~mL}$ of $\mathrm{Et}_{2} \mathrm{O}$, and the combined organic layers were 
washed with brine $(40 \mathrm{~mL})$, dried $\left(\mathrm{MgSO}_{4}\right)$, and concentrated in vacuo. Purification by flash chromatography (1:99 to 5:95 $\mathrm{Et}_{2} \mathrm{O} /$ hexanes) afforded 26 as a colorless oil $(12.0 \mathrm{~g}, 96 \%):{ }^{1} \mathrm{H} \mathrm{NMR}\left(\mathrm{CDCl}_{3}, 500 \mathrm{MHz}\right) \delta$ $7.48-7.19(\mathrm{~m}, 15 \mathrm{H}), 4.58(\mathrm{~d}, J=11.6 \mathrm{~Hz}, 1 \mathrm{H}), 4.50(\mathrm{~d}, J=12.1 \mathrm{~Hz}, 1 \mathrm{H}), 4.44(\mathrm{~d}, J=12.1 \mathrm{~Hz}, 1 \mathrm{H}), 4.18(\mathrm{~d}, J=$ $11.6 \mathrm{~Hz}, 1 \mathrm{H}), 3.97(\mathrm{~s}, 1 \mathrm{H}), 3.52(\mathrm{dd}, J=10.0,1.4 \mathrm{~Hz}, 1 \mathrm{H}), 3.22(\mathrm{~d}, J=9.2 \mathrm{~Hz}, 1 \mathrm{H}), 3.11(\mathrm{~d}, J=9.2 \mathrm{~Hz}, 1 \mathrm{H})$, $1.37(\mathrm{~m}, 1 \mathrm{H}), 1.23\left(\mathrm{~m}, 1 \mathrm{H}\right.$ and s, 3H), $1.07(\mathrm{~s}, 3 \mathrm{H}), 0.92(\mathrm{t}, J=7.3 \mathrm{~Hz}, 3 \mathrm{H}), 0.30(\mathrm{~s}, 3 \mathrm{H}), 0.29(\mathrm{~s}, 3 \mathrm{H}) ;{ }^{13} \mathrm{C} \mathrm{NMR}$ $\left(\mathrm{CDCl}_{3}, 125 \mathrm{MHz}\right) \delta 138.9,138.5,137.0,134.4,129.1,128.3,128.1,127.64,127.56,127.5,127.14,127.11$, 86.3, 82.0, 81.4, 76.1, 73.4, 72.0, 37.5, 24.0, 20.7, 12.2, 9.8, -4.8, -5.1; IR (thin film) 2960, $1453,1110 \mathrm{~cm}^{-1}$; $[\alpha]^{23}{ }_{D}+15.4\left(c\right.$ 1.04, $\mathrm{CHCl}_{3}$ ); HRMS (ESI) $m / z$ calcd for $\mathrm{C}_{31} \mathrm{H}_{40} \mathrm{O}_{3} \mathrm{SiNa}(\mathrm{M}+\mathrm{Na})^{+}$511.2644, found 511.2630 . Anal. Calcd for $\mathrm{C}_{31} \mathrm{H}_{40} \mathrm{O}_{3} \mathrm{Si}$ : C, 76.19; H, 8.26. Found: $\mathrm{C}, 76.31 ; \mathrm{H}, 8.31$.

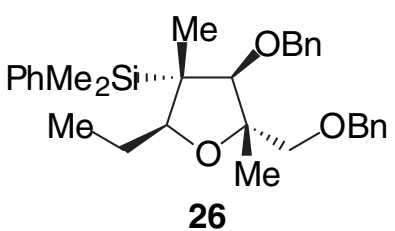

26

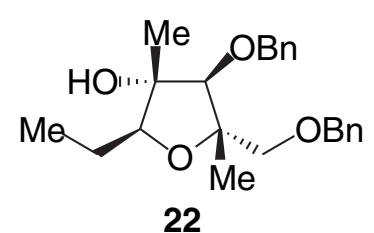

22

(2S,3S,4S,5S)-3-Benzyloxy-2-benzyloxymethyl-2,4-dimethyl-5-ethyl-4-hydroxytetrahydrofuran (22). To a suspension of $\mathrm{KH}(7.20 \mathrm{~g}, 180 \mathrm{mmol})$ in $100 \mathrm{~mL}$ of NMP was added cumene hydroperoxide $(25.1 \mathrm{~mL}, 88.0 \%$, $150 \mathrm{mmol})$. After $20 \mathrm{~min}$, silane $26(14.6 \mathrm{~g}, 30.0 \mathrm{mmol})$ in $100 \mathrm{~mL}$ of NMP was added dropwise followed by $n$-Bu $\mathrm{Bu}_{4} \mathrm{NF}(60.0 \mathrm{~mL}, 1 \mathrm{M}$ solution in THF, $60.0 \mathrm{mmol})$. After $40 \mathrm{~h}$ at $23{ }^{\circ} \mathrm{C}, 100 \mathrm{~mL}$ of aqueous saturated $\mathrm{Na}_{2} \mathrm{~S}_{2} \mathrm{O}_{3}$ solution was added, and the mixture was stirred for $30 \mathrm{~min}$ at $23{ }^{\circ} \mathrm{C}$. The mixture was then extracted with $5 \times 200 \mathrm{~mL}$ of $t$-BuOMe. The combined organic layers were washed with brine $(50 \mathrm{~mL}), \operatorname{dried}\left(\mathrm{MgSO}_{4}\right)$, and concentrated in vacuo. Purification by flash chromatography (5:95 to 30:70 EtOAc/hexanes) afforded 22 $(9.40 \mathrm{~g}, 85 \%)$ as a colorless oil: ${ }^{1} \mathrm{H}$ NMR $\left(\mathrm{CDCl}_{3}, 500 \mathrm{MHz}\right) \delta 7.30(\mathrm{~m}, 10 \mathrm{H}), 4.69(\mathrm{~d}, J=12.0 \mathrm{~Hz}, 1 \mathrm{H}), 4.67$ $(\mathrm{d}, J=12.0 \mathrm{~Hz}, 1 \mathrm{H}), 4.64(\mathrm{~d}, J=12.0 \mathrm{~Hz}, 1 \mathrm{H}), 4.59(\mathrm{~d}, J=12.0 \mathrm{~Hz}, 1 \mathrm{H}), 3.91(\mathrm{~s}, 1 \mathrm{H}), 3.66(\mathrm{dd}, J=9.2,4.7 \mathrm{~Hz}$, $1 \mathrm{H}), 3.49(\mathrm{~d}, J=9.6 \mathrm{~Hz}, 1 \mathrm{H}), 3.41(\mathrm{~d}, J=9.6 \mathrm{~Hz}, 1 \mathrm{H}), 2.95(\mathrm{~s}, 1 \mathrm{H}), 1.54(\mathrm{~m}, 2 \mathrm{H}), 1.32(\mathrm{~s}, 3 \mathrm{H}), 1.25(\mathrm{~s}, 3 \mathrm{H})$, $1.04(\mathrm{t}, J=7.4 \mathrm{~Hz}, 3 \mathrm{H}) ;{ }^{13} \mathrm{C}$ NMR $\left(\mathrm{CDCl}_{3}, 125 \mathrm{MHz}\right) \delta 138.5,137.8,128.4,128.3,127.7,127.6,127.5,127.4$, 88.8, 85.4, 82.0, 81.0, 76.6, 73.6, 73.3, 23.0, 20.3, 16.8, 10.7; IR (thin film) 3445, $1453 \mathrm{~cm}^{-1} ;[\alpha]_{\mathrm{D}}^{23}-39.1(c$ 0.92, $\left.\mathrm{CHCl}_{3}\right)$; HRMS $\left(\mathrm{CI} / \mathrm{NH}_{3}\right) \mathrm{m} / \mathrm{z}$ calcd for $\mathrm{C}_{15} \mathrm{H}_{21} \mathrm{O}_{3}\left(\mathrm{M}-\mathrm{BnOCH}_{2}\right)^{+}$249.1491, found 249.1490. Anal. Calcd for $\mathrm{C}_{23} \mathrm{H}_{30} \mathrm{O}_{4}: \mathrm{C}, 74.55 ; \mathrm{H}, 8.17$. Found: $\mathrm{C}, 74.31 ; \mathrm{H}, 8.20$.
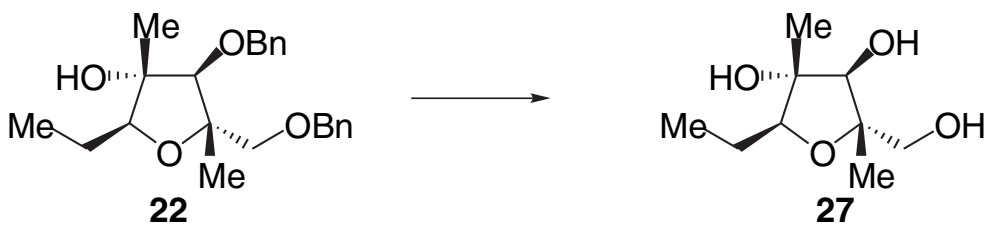

(2S,3S,4S,5S)-2,4-Dimethyl-3,4-dihydroxy-5-ethyl-2-hydroxymethyltetrahydrofuran (27). Dibenzyl ether $22(1.39 \mathrm{~g}, 3.76 \mathrm{mmol})$ was dissolved in $30 \mathrm{~mL}$ of EtOH, then $10 \% \mathrm{Pd}-\mathrm{C}(0.300 \mathrm{~g})$ was added, and the mixture was hydrogenated with $\mathrm{H}_{2}$ supplied by a balloon $(1 \mathrm{~atm})$. After $24 \mathrm{~h}$, the reaction mixture was filtered, and the filtrate was concentrated in vacuo. Purification by flash chromatography (5:95 to $10: 90 \mathrm{MeOH} / \mathrm{CH}_{2} \mathrm{Cl}_{2}$ ) afforded 27 as a colorless oil $(0.700 \mathrm{~g}, 98 \%):{ }^{1} \mathrm{H} \mathrm{NMR}\left(\mathrm{CDCl}_{3}, 500 \mathrm{MHz}\right) \delta 4.07(\mathrm{~d}, J=5.3 \mathrm{~Hz}, 1 \mathrm{H}), 3.61(\mathrm{t}, J$ $=6.9 \mathrm{~Hz}, 1 \mathrm{H}), 3.56(\mathrm{~m}, 2 \mathrm{H}), 3.08(\mathrm{br}, 1 \mathrm{H}), 2.61(\mathrm{br}, 1 \mathrm{H}), 2.35(\mathrm{br}, 1 \mathrm{H}), 1.53$ (quintet, $J=7.3 \mathrm{~Hz}, 2 \mathrm{H}), 1.25(\mathrm{~s}$, $3 \mathrm{H}), 1.16(\mathrm{~s}, 3 \mathrm{H}), 1.00(\mathrm{t}, J=7.4 \mathrm{~Hz}, 3 \mathrm{H}) ;{ }^{13} \mathrm{C} \mathrm{NMR}\left(\mathrm{CDCl}_{3}, 125 \mathrm{MHz}\right) \delta 84.8,82.1,80.7,80.6,68.5,23.1$, 19.3, 16.1, 10.7; IR (thin film) 3424, $1047 \mathrm{~cm}^{-1} ;[\alpha]_{\mathrm{D}}^{23}-50.7$ (c 1.14, $\left.\mathrm{CHCl}_{3}\right) ; \mathrm{HRMS}\left(\mathrm{CI} / \mathrm{NH}_{3}\right) \mathrm{m} / z$ calcd for $\mathrm{C}_{9} \mathrm{H}_{19} \mathrm{O}_{3}(\mathrm{M}+\mathrm{H})^{+}$191.1283, found 191.1285. 

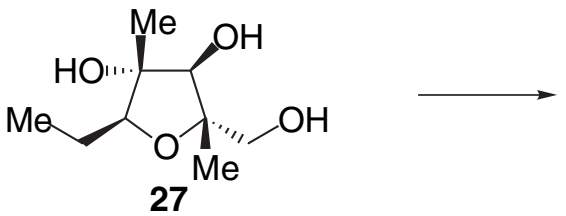

(2S,3S,4S,5S)-2,4-Dimethyl-3,4-dihydroxy-5-ethyl-2-triisopropylsiloxymethyltetrahydrofuran (28). To triol 27 (0.333 g, $1.75 \mathrm{mmol})$ in $8 \mathrm{~mL}$ of dry DMF was added imidazole $(0.143 \mathrm{~g}, 2.10 \mathrm{mmol})$ and TIPSCl $(0.450 \mathrm{~mL}, 2.10 \mathrm{mmol})$. After $12 \mathrm{~h}$ at $23{ }^{\circ} \mathrm{C}, 10 \mathrm{~mL}$ of saturated aqueous $\mathrm{NH}_{4} \mathrm{Cl}$ was added and the solution was extracted with $3 \times 30 \mathrm{~mL}$ of $\mathrm{Et}_{2} \mathrm{O}$. The combined organic layers were washed with brine $(10 \mathrm{~mL})$, dried $\left(\mathrm{MgSO}_{4}\right)$, and concentrated in vacuo. Purification by flash chromatography (15:85 to 20:80 EtOAc/hexanes) afforded 28 as a white solid $(0.583 \mathrm{~g}, 96 \%)$ : $\mathrm{mp} 102-103{ }^{\circ} \mathrm{C} ;{ }^{1} \mathrm{H}$ NMR $\left(\mathrm{CDCl}_{3}, 500 \mathrm{MHz}\right) \delta 4.17(\mathrm{~d}, J=4.8 \mathrm{~Hz}$, $1 \mathrm{H}), 3.68(\mathrm{~d}, J=9.7 \mathrm{~Hz}, 1 \mathrm{H}), 3.66(\mathrm{dd}, J=7.9,2.0 \mathrm{~Hz}, 1 \mathrm{H}), 3.63(J=9.7 \mathrm{~Hz}, 1 \mathrm{H}), 2.87(\mathrm{~s}, 1 \mathrm{H}), 1.72(\mathrm{~d}, J=4.8$ $\mathrm{Hz}, 1 \mathrm{H}), 1.50(\mathrm{~m}, 2 \mathrm{H}), 1.24(\mathrm{~s}, 3 \mathrm{H}), 1.16(\mathrm{~s}, 3 \mathrm{H}), 1.09(\mathrm{~m}, 21 \mathrm{H}), 0.99(\mathrm{t}, J=7.4 \mathrm{~Hz}, 3 \mathrm{H}) ;{ }^{13} \mathrm{C} \mathrm{NMR}\left(\mathrm{CDCl}_{3}, 125\right.$ $\mathrm{MHz}$ ) 85.1, 82.2, 81.9, 80.3, 70.3, 23.2, 19.5, 18.0, 16.2, 11.9, 10.9; IR (thin film) 3326, 1460, $1094 \mathrm{~cm}^{-1} ;[\alpha]^{23}{ }_{\mathrm{D}}$ -35.8 ( c 1.65, $\left.\mathrm{CHCl}_{3}\right)$; HRMS $\left(\mathrm{CI} / \mathrm{NH}_{3}\right) \mathrm{m} / \mathrm{z}$ calcd for $\mathrm{C}_{18} \mathrm{H}_{39} \mathrm{O}_{4} \mathrm{Si}(\mathrm{M}+\mathrm{H})^{+}$347.2617, found 347.2621. Anal. Calcd for $\mathrm{C}_{18} \mathrm{H}_{38} \mathrm{O}_{4} \mathrm{Si}$ : C, 62.38; H, 11.05. Found: C, 62.52; H, 11.19.
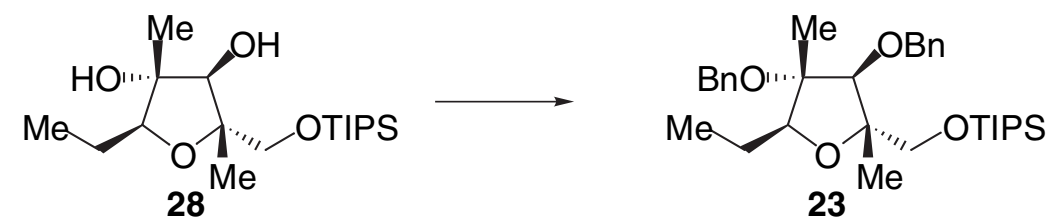

(2S,3S,4S,5S)-3,4-Dibenzyloxy-2,4-dimethyl-5-ethyl-2-triisopropylsiloxymethyltetrahydrofuran (23). To a suspension of $\mathrm{NaH}(0.800 \mathrm{~g}, 60 \%$ oil dispersion, $20.2 \mathrm{mmol}$, washed with $3 \times 20 \mathrm{~mL}$ of hexanes $)$ in $20 \mathrm{~mL}$ of DMF was added a solution of diol $28(1.40 \mathrm{~g}, 4.05 \mathrm{mmol})$ in $20 \mathrm{~mL}$ of DMF at $0{ }^{\circ} \mathrm{C}$. After $0.5 \mathrm{~h}, \mathrm{BnBr}(2.41$ $\mathrm{mL}, 20.2 \mathrm{mmol})$ and $n-\mathrm{Bu}_{4} \mathrm{NI}(0.074 \mathrm{~g}, 0.200 \mathrm{mmol})$ were added at $0{ }^{\circ} \mathrm{C}$, and the reaction mixture was allowed to warm to $23{ }^{\circ} \mathrm{C}$. After $12 \mathrm{~h}$, a solution of saturated aqueous $\mathrm{NH}_{4} \mathrm{Cl}(40 \mathrm{~mL})$ was added. The mixture was extracted with $3 \times 100 \mathrm{~mL}$ of $\mathrm{Et}_{2} \mathrm{O}$, and the combined organic layers were washed with brine $(40 \mathrm{~mL})$, dried $\left(\mathrm{MgSO}_{4}\right)$, and concentrated in vacuo. Purification by flash chromatography (1:99 $\mathrm{Et}_{2} \mathrm{O}$ /hexanes) afforded 23 as a colorless oil $(2.05 \mathrm{~g}, 96 \%):{ }^{1} \mathrm{H} \mathrm{NMR}\left(\mathrm{CDCl}_{3}, 500 \mathrm{MHz}\right) \delta 7.44-7.31(\mathrm{~m}, 10 \mathrm{H}), 4.79(\mathrm{~d}, J=11.8 \mathrm{~Hz}, 1 \mathrm{H}), 4.70$ $(\mathrm{d}, J=11.7 \mathrm{~Hz}, 1 \mathrm{H}), 4.57(\mathrm{~d}, J=11.8 \mathrm{~Hz}, 1 \mathrm{H}), 4.55(\mathrm{~d}, J=11.7 \mathrm{~Hz}, 1 \mathrm{H}), 4.35(\mathrm{~s}, 1 \mathrm{H}), 3.87(\mathrm{dd}, J=9.0,3.9 \mathrm{~Hz}$, $1 \mathrm{H}), 3.82(\mathrm{~d}, J=9.6 \mathrm{~Hz}, 1 \mathrm{H}), 3.70(J=9.6 \mathrm{~Hz}, 1 \mathrm{H}), 1.67(\mathrm{~m}, 1 \mathrm{H}), 1.57(\mathrm{~m}, 1 \mathrm{H}), 1.43(\mathrm{~s}, 3 \mathrm{H}), 1.30(\mathrm{~s}, 3 \mathrm{H}), 1.19$ $(\mathrm{m}, 21 \mathrm{H}), 1.08(\mathrm{t}, J=7.5 \mathrm{~Hz}, 3 \mathrm{H}) ;{ }^{13} \mathrm{C} \mathrm{NMR}\left(\mathrm{CDCl}_{3}, 125 \mathrm{MHz}\right) 139.5,138.7,128.3,128.2,127.4,127.3$, $127.12,127.10,87.3,84.9,82.04,82.01,72.2,70.1,65.5,22.8,19.7,18.1,13.2,12.0,11.0$; IR (thin film) 1462 , $1098 \mathrm{~cm}^{-1} ;[\alpha]_{D}^{23}+19.0\left(c 1.01, \mathrm{CHCl}_{3}\right)$; HRMS $\left(\mathrm{CI} / \mathrm{NH}_{3}\right) \mathrm{m} / \mathrm{z}$ calcd for $\mathrm{C}_{32} \mathrm{H}_{51} \mathrm{O}_{4} \mathrm{Si}(\mathrm{M}+\mathrm{H})^{+} 527.3556$, found 527.3554. Anal. Calcd for $\mathrm{C}_{32} \mathrm{H}_{50} \mathrm{O}_{4} \mathrm{Si}: \mathrm{C}, 72.95 ; \mathrm{H}, 9.57$. Found: $\mathrm{C}, 73.12 ; \mathrm{H}, 9.59$. 


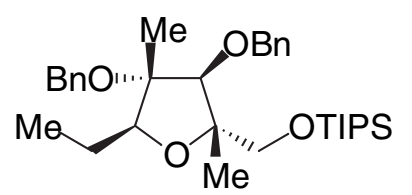

23

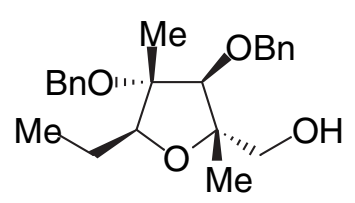

29

(2S,3S,4S,5S)-3,4-Dibenzyloxy-2,4-dimethyl-5-ethyl-2-hydroxymethyltetrahydrofuran (29). To a solution of $23(0.130 \mathrm{~g}, 0.250 \mathrm{mmol})$ in $3 \mathrm{~mL}$ of dry THF was added $n-\mathrm{Bu}_{4} \mathrm{NF}(1.00 \mathrm{M}$ solution in THF, $0.375 \mathrm{~mL}$, $0.375 \mathrm{mmol})$. After stirring for $12 \mathrm{~h}$ at $23{ }^{\circ} \mathrm{C}$, saturated aqueous $\mathrm{NH}_{4} \mathrm{Cl}$ solution $(5 \mathrm{~mL})$ was added. The mixture was extracted with $3 \times 20 \mathrm{~mL}$ of $\mathrm{Et}_{2} \mathrm{O}$. The combined organic layers were washed with $10 \mathrm{~mL}$ of brine, dried $\left(\mathrm{MgSO}_{4}\right)$, filtered, and concentrated in vacuo. Purification by flash chromatography (15:85 to 20:80 EtOAc/hexanes) afforded 29 as a colorless oil $(0.090 \mathrm{~g}, 97 \%):{ }^{1} \mathrm{H}$ NMR $\left(\mathrm{CDCl}_{3}, 500 \mathrm{MHz}\right) \delta 7.47-7.32(\mathrm{~m}$, $10 \mathrm{H}), 4.72(\mathrm{~d}, J=11.6 \mathrm{~Hz}, 1 \mathrm{H}), 4.70(\mathrm{~d}, J=11.5 \mathrm{~Hz}, 1 \mathrm{H}), 4.63(\mathrm{~d}, J=11.6 \mathrm{~Hz}, 1 \mathrm{H}), 4.56(\mathrm{~d}, J=11.5 \mathrm{~Hz}, 1 \mathrm{H})$, $4.13(\mathrm{~s}, 1 \mathrm{H}), 3.83(\mathrm{dd}, J=8.9,4.2 \mathrm{~Hz}, 1 \mathrm{H}), 3.71(\mathrm{~d}, J=11.1 \mathrm{~Hz}, 1 \mathrm{H}), 3.59(J=11.1 \mathrm{~Hz}, 1 \mathrm{H}), 2.50(\mathrm{br}, 1 \mathrm{H})$, $1.70(\mathrm{~m}, 2 \mathrm{H}), 1.47(\mathrm{~s}, 3 \mathrm{H}), 1.35(\mathrm{~s}, 3 \mathrm{H}), 1.12(\mathrm{t}, J=7.5 \mathrm{~Hz}, 3 \mathrm{H}) ;{ }^{13} \mathrm{C}$ NMR $\left(\mathrm{CDCl}_{3}, 125 \mathrm{MHz}\right)$ 139.0, 138.0, $128.3,128.2,127.7,127.5,127.2,127.1,87.2,84.8,82.1,82.0,72.6,69.0,65.5,22.9,19.2,13.2$, 10.8; IR (thin

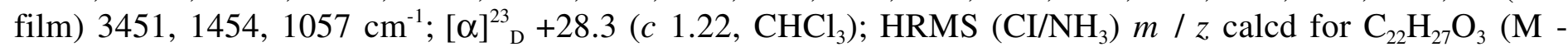
$\left.\mathrm{CH}_{2} \mathrm{OH}\right)^{+}$339.1960, found 339.1963. Anal. Calcd for $\mathrm{C}_{23} \mathrm{H}_{30} \mathrm{O}_{4}: \mathrm{C}, 74.56 ; \mathrm{H}$, 8.16. Found: C, 74.30; H, 8.35.

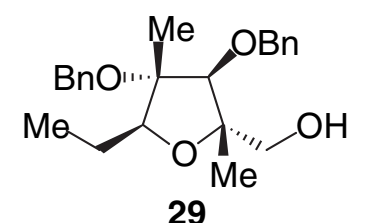

29

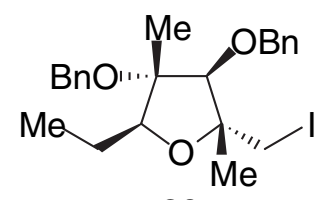

30

(2S,3S,4S,5S)-3,4-Dibenzyloxy-2,4-dimethyl-5-ethyl-2-iodomethyltetrahydrofuran (30). To a solution of 29 (1.47 g, $3.97 \mathrm{mmol})$ in $40 \mathrm{~mL}$ of benzene was added $\mathrm{Ph}_{3} \mathrm{P}(1.25 \mathrm{~g}, 4.77 \mathrm{mmol})$, imidazole $(0.650 \mathrm{~g}, 9.54$ mmol), and $\mathrm{I}_{2}(1.21 \mathrm{~g}, 4.77 \mathrm{mmol})$. The mixture was heated at reflux for $0.5 \mathrm{~h}$. After being cooled to $23{ }^{\circ} \mathrm{C}$, the mixture was filtered through Celite and washed with ether. The filtrate was concentrated in vacuo. Purification by flash chromatography (5:95 EtOAc/hexanes) afforded 30 as a colorless oil $(1.23 \mathrm{~g}, 65 \%)$ : ${ }^{1} \mathrm{H} \mathrm{NMR}\left(\mathrm{CDCl}_{3}\right.$, $500 \mathrm{MHz}) \delta 7.49-7.32(\mathrm{~m}, 10 \mathrm{H}), 4.83(\mathrm{~d}, J=11.5 \mathrm{~Hz}, 1 \mathrm{H}), 4.69(\mathrm{~d}, J=11.5 \mathrm{~Hz}, 1 \mathrm{H}), 4.65(\mathrm{~d}, J=11.4 \mathrm{~Hz}, 1 \mathrm{H})$, $4.52(\mathrm{~d}, J=11.4 \mathrm{~Hz}, 1 \mathrm{H}), 4.18(\mathrm{~s}, 1 \mathrm{H}), 3.90(\mathrm{dd}, J=8.2,4.9 \mathrm{~Hz}, 1 \mathrm{H}), 3.52(\mathrm{~d}, J=10.1 \mathrm{~Hz}, 1 \mathrm{H}), 3.49(J=10.1$ $\mathrm{Hz}, 1 \mathrm{H}), 1.64(\mathrm{~m}, 2 \mathrm{H}), 1.49(\mathrm{~s}, 3 \mathrm{H}), 1.42(\mathrm{~s}, 3 \mathrm{H}), 1.11(\mathrm{t}, J=7.4 \mathrm{~Hz}, 3 \mathrm{H}) ;{ }^{13} \mathrm{C} \mathrm{NMR}\left(\mathrm{CDCl}_{3}, 125 \mathrm{MHz}\right) 138.9$, 138.0, 128.4, 128.3, 127.7, 127.6, 127.3, 127.1, 89.1, 86.4, 82.5, 81.0, 73.0, 65.5, 23.1, 22.6, 17.7, 13.2, 10.9; IR (thin film) 1455, $1105 \mathrm{~cm}^{-1} ;[\alpha]^{23}+33.1\left(c\right.$ 1.11, $\left.\mathrm{CHCl}_{3}\right) ; \mathrm{HRMS}\left(\mathrm{CI} / \mathrm{NH}_{3}\right) \mathrm{m} / z$ calcd for $\mathrm{C}_{23} \mathrm{H}_{33} \mathrm{IO}_{3} \mathrm{~N}\left(\mathrm{M}_{+}+\right.$ $\left.\mathrm{NH}_{4}\right)^{+}$498.1981, found 498.1973. Anal. Calcd for $\mathrm{C}_{23} \mathrm{H}_{29} \mathrm{IO}_{3}: \mathrm{C}, 57.51 ; \mathrm{H}, 6.08$. Found: C, 57.78; H, 6.25.

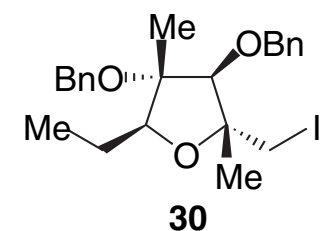

30

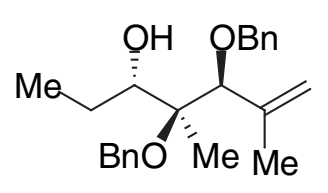

31

(3R,4S,5R)-3,4-Dibenzyloxy-2,4-dimethylhept-1-en-5-ol (31). To a solution of the iodide 30 (1.20 g, 2.50 $\mathrm{mmol})$ in $10 \mathrm{~mL}$ of $\mathrm{Et}_{2} \mathrm{O}$ and $10 \mathrm{~mL}$ of $\mathrm{MeOH}$ was added zinc powder $(1.63 \mathrm{~g}, 25.0 \mathrm{mmol})$ followed by $\mathrm{AcOH}$ 
$(0.310 \mathrm{~mL}, 5.00 \mathrm{mmol})$. The reaction mixture was stirred for $12 \mathrm{~h}$ at $23{ }^{\circ} \mathrm{C}$, at which point the reaction mixture was diluted with $100 \mathrm{~mL}$ of $\mathrm{Et}_{2} \mathrm{O}$ and filtered. The filtrate was washed with $30 \mathrm{~mL}$ of saturated aqueous $\mathrm{NaHCO}_{3}$ and brine $(20 \mathrm{~mL})$, then dried $\left(\mathrm{MgSO}_{4}\right)$ and concentrated in vacuo. Purification by flash chromatography (10:90 EtOAc/hexanes) afforded 31 as a colorless oil $(0.840 \mathrm{~g}, 95 \%):{ }^{1} \mathrm{H}$ NMR $\left(\mathrm{CDCl}_{3}, 500\right.$ MHz) $\delta 7.47-7.32(\mathrm{~m}, 10 \mathrm{H}), 5.30(\mathrm{t}, J=1.6 \mathrm{~Hz}, 1 \mathrm{H}), 5.12(\mathrm{~s}, 1 \mathrm{H}), 4.82(\mathrm{~d}, J=11.3 \mathrm{~Hz}, 1 \mathrm{H}), 4.69(\mathrm{~d}, J=11.6$ $\mathrm{Hz}, 1 \mathrm{H}), 4.63(\mathrm{~d}, J=11.3 \mathrm{~Hz}, 1 \mathrm{H}), 4.34(\mathrm{~d}, J=11.6 \mathrm{~Hz}, 1 \mathrm{H}), 4.10(\mathrm{~s}, 1 \mathrm{H}), 3.84(\mathrm{ddd}, J=10.5,6.5,1.8 \mathrm{~Hz}, 1 \mathrm{H})$, $3.44(\mathrm{~d}, J=6.5 \mathrm{~Hz}, 1 \mathrm{H}), 2.00(\mathrm{~s}, 3 \mathrm{H}), 1.66(\mathrm{~m}, 1 \mathrm{H}), 1.33(\mathrm{~s}, 3 \mathrm{H}$ and $\mathrm{m}, 1 \mathrm{H}), 1.14(\mathrm{t}, J=7.3 \mathrm{~Hz}, 3 \mathrm{H}) ;{ }^{13} \mathrm{C} \mathrm{NMR}$ $\left(\mathrm{CDCl}_{3}, 125 \mathrm{MHz}\right) 142.2,139.4,137.4,128.4,128.2,128.1,127.8,127.2,126.9,117.0,87.2,80.1,76.2,70.5$, 64.2, 24.9, 19.6, 18.2, 11.4; IR (thin film) 3500, 1450, $1061 \mathrm{~cm}^{-1} ;[\alpha]_{\mathrm{D}}^{23}+33.2\left(c 1.04, \mathrm{CHCl}_{3}\right) ; \mathrm{HRMS}$ $\left(\mathrm{CI} / \mathrm{NH}_{3}\right) m / z$ calcd for $\mathrm{C}_{23} \mathrm{H}_{31} \mathrm{O}_{3}(\mathrm{M}+\mathrm{H})^{+}$355.2273, found 355.2285. Anal. Calcd for $\mathrm{C}_{23} \mathrm{H}_{30} \mathrm{O}_{3}: \mathrm{C}, 77.93 ; \mathrm{H}$, 8.53. Found: $\mathrm{C}, 77.81 ; \mathrm{H}, 8.45$.<smiles>C=C(C)[C@H](OCc1ccccc1)[C@]([14CH3])(Br)[C@H](O)CC</smiles>

31

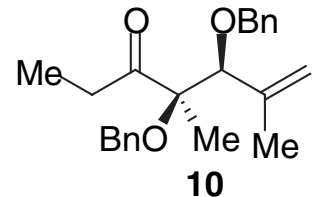

10

(3R,4S,5R)-4,5-Dibenzyloxy-4,6-dimethylhept-6-en-3-one (10). To a solution of oxalyl chloride $(0.350 \mathrm{~mL}$, $2.0 \mathrm{M}$ solution in $\left.\mathrm{CH}_{2} \mathrm{Cl}_{2}, 0.700 \mathrm{mmol}\right)$ in $4 \mathrm{~mL}$ of $\mathrm{CH}_{2} \mathrm{Cl}_{2}$ at $-78{ }^{\circ} \mathrm{C}$ was added a solution of DMSO $(0.099 \mathrm{~mL}$, $1.39 \mathrm{mmol}$ ) in $0.5 \mathrm{~mL}$ of $\mathrm{CH}_{2} \mathrm{Cl}_{2}$. The mixture was stirred at $-78{ }^{\circ} \mathrm{C}$ for $10 \mathrm{~min}$, and then a solution of alcohol $31(0.205 \mathrm{~g}, 0.580 \mathrm{mmol})$ in $2 \mathrm{~mL}$ of $\mathrm{CH}_{2} \mathrm{Cl}_{2}$ was added dropwise by syringe. After $1 \mathrm{~h}$ at $-78{ }^{\circ} \mathrm{C}, 0.400 \mathrm{~mL}$ ( $2.90 \mathrm{mmol}$ ) of $\mathrm{Et}_{3} \mathrm{~N}$ was added. The mixture was allowed to warm to $23{ }^{\circ} \mathrm{C}$ and was then poured into $10 \mathrm{~mL}$ of water. The layers were separated, and the aqueous layer was extracted with $3 \times 30 \mathrm{~mL}$ of $\mathrm{CH}_{2} \mathrm{Cl}_{2}$. The combined organic layers were washed with $1 \mathrm{~N} \mathrm{HCl}(10 \mathrm{~mL})$, aqueous $\mathrm{NaHCO}_{3}(10 \mathrm{~mL})$, brine $(10 \mathrm{~mL})$, dried $\left(\mathrm{MgSO}_{4}\right)$, and concentrated in vacuo. Purification by flash chromatography (3:97 to 5:95 EtOAc/hexanes) afforded 10 as a colorless oil $(0.196 \mathrm{~g}, 96 \%):{ }^{1} \mathrm{H}$ NMR $\left(\mathrm{CDCl}_{3}, 500 \mathrm{MHz}\right) \delta 7.51-7.37(\mathrm{~m}, 10 \mathrm{H}), 5.27(\mathrm{t}, J=1.6$ $\mathrm{Hz}, 1 \mathrm{H}), 5.09$ (s, 1H), 4.69 (d, $J=11.9 \mathrm{~Hz}, 1 \mathrm{H}), 4.64(\mathrm{~d}, J=11.8 \mathrm{~Hz}, 1 \mathrm{H}), 4.63(\mathrm{~d}, J=11.9 \mathrm{~Hz}, 1 \mathrm{H}), 4.32(\mathrm{~d}, J$ $=11.8 \mathrm{~Hz}, 1 \mathrm{H}), 4.07(\mathrm{~s}, 1 \mathrm{H}), 3.00(\mathrm{dq}, J=18.7,7.2 \mathrm{~Hz}, 1 \mathrm{H}), 2.60(\mathrm{dq}, J=18.7,7.2 \mathrm{~Hz}, 1 \mathrm{H}), 1.99(\mathrm{~s}, 3 \mathrm{H}), 1.49$ $(\mathrm{s}, 3 \mathrm{H}), 1.12(\mathrm{t}, J=7.2 \mathrm{~Hz}, 3 \mathrm{H}) ;{ }^{13} \mathrm{C} \mathrm{NMR}\left(\mathrm{CDCl}_{3}, 125 \mathrm{MHz}\right) 215.0,141.7,138.7,137.9,128.2,128.1,127.7$, 127.4, 127.1, 126.8, 117.6, 88.2, 87.4, 70.5, 67.0, 32.3, 18.8, 18.2, 7.5; IR (thin film) 1711, 1455, $1088 \mathrm{~cm}^{-1}$; $[\alpha]^{23}{ }_{D}+65.0\left(c\right.$ 1.29, $\left.\mathrm{CHCl}_{3}\right)$; HRMS $\left(\mathrm{CI} / \mathrm{NH}_{3}\right) \mathrm{m} / \mathrm{z}$ calcd for $\mathrm{C}_{23} \mathrm{H}_{30} \mathrm{O}_{4}\left(\mathrm{M}+\mathrm{NH}_{4}\right)^{+} 370.2382$, found 370.2385 . Anal. Calcd for $\mathrm{C}_{23} \mathrm{H}_{28} \mathrm{O}_{3}: \mathrm{C}, 78.38 ; \mathrm{H}, 8.01$. Found: C, 78.31; H, 8.09.

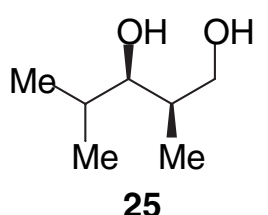

25

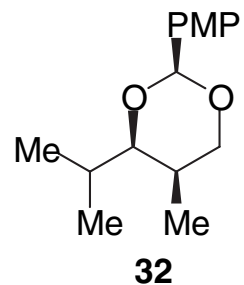

(2S,4R,5R)-4-Isopropyl-2-( $p$-methoxyphenyl)-5-methyl-1,3-dioxane (32). To a solution of $\mathbf{2 5}^{3}$ (0.500 g, 3.82 $\mathrm{mmol})$ in $20 \mathrm{~mL}$ of $\mathrm{CH}_{2} \mathrm{Cl}_{2}$ was added $p$-anisaldehyde dimethylacetal $(0.720 \mathrm{~mL}, 4.20 \mathrm{mmol})$ and CSA (0.044 $\mathrm{g}, 0.190 \mathrm{mmol})$. After $6 \mathrm{~h}$ at $23{ }^{\circ} \mathrm{C}, 0.2 \mathrm{~mL}$ of $\mathrm{Et}_{3} \mathrm{~N}$ was added to the reaction mixture. The mixture was then concentrated in vасио. Purification by flash chromatography (5:95 EtOAc/hexanes) afforded $\mathbf{3 2}$ as a colorless

\footnotetext{
${ }^{3}$ Crimmins, M. T.; King, B. W.; Tabet, E. A.; Chaudhary, K. J. Org. Chem. 2001, 66, 894-902.
} 
oil $(0.930 \mathrm{~g}, 97 \%):{ }^{1} \mathrm{H} \mathrm{NMR}\left(\mathrm{CDCl}_{3}, 500 \mathrm{MHz}\right) \delta 7.48(\mathrm{dt}, J=8.7,2.2 \mathrm{~Hz}, 2 \mathrm{H}), 6.93(\mathrm{dt}, J=8.7,2.4 \mathrm{~Hz}, 2 \mathrm{H})$, $5.48(\mathrm{~s}, 1 \mathrm{H}), 4.06(\mathrm{AB}, 2 \mathrm{H}), 3.82(\mathrm{~s}, 3 \mathrm{H}), 3.40(\mathrm{dd}, J=9.9,2.2 \mathrm{~Hz}, 1 \mathrm{H}), 1.83(\mathrm{~m}, 1 \mathrm{H}), 1.70(\mathrm{~m}, 1 \mathrm{H}), 1.20(\mathrm{~d}, J$ $=6.9 \mathrm{~Hz}, 3 \mathrm{H}), 1.07(\mathrm{~d}, J=6.5 \mathrm{~Hz}, 3 \mathrm{H}), 0.90(\mathrm{~d}, J=6.8 \mathrm{~Hz}, 3 \mathrm{H}) ;{ }^{13} \mathrm{C} \mathrm{NMR}\left(\mathrm{CDCl}_{3}, 125 \mathrm{MHz}\right) 159.6,131.7$, 127.1, 113.4, 101.5, 85.6, 73.8, 55.1, 29.9, 29.4, 19.5, 17.3, 10.9; IR (thin film) 2960, 1616, $1518 \mathrm{~cm}^{-1}$; $[\alpha]^{23}{ }_{\mathrm{D}}$ +30.6 (c 1.29, $\left.\mathrm{CHCl}_{3}\right)$; HRMS (CI/ $\left.\mathrm{NH}_{3}\right) m / z$ calcd for $\mathrm{C}_{15} \mathrm{H}_{21} \mathrm{O}_{3}(\mathrm{M}-\mathrm{H})^{+}$249.1491, found 249.1487. Anal. Calcd for $\mathrm{C}_{15} \mathrm{H}_{22} \mathrm{O}_{3}$ : C, 71.97; $\mathrm{H}, 8.86$. Found: $\mathrm{C}, 72.05 ; \mathrm{H}, 8.87$.<smiles>CC(C)[C@H]1OC([18OH])OC[C@H]1C</smiles>

32

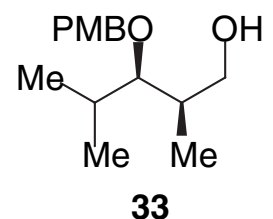

33

(2S,3R)-2,4-Dimethyl-3-(p-methoxybenzyloxy)-pentan-1-ol (33). To a solution of $\mathbf{3 2}(0.920 \mathrm{~g}, 3.69 \mathrm{mmol})$ in $30 \mathrm{~mL}$ of $\mathrm{CH}_{2} \mathrm{Cl}_{2}$ was added $i-\mathrm{Bu}_{2} \mathrm{AlH}(5.38 \mathrm{~mL}, 1.50 \mathrm{M}$ solution in toluene, $8.07 \mathrm{mmol})$ dropwise at $-78{ }^{\circ} \mathrm{C}$. The reaction mixture was allowed to warm to $0{ }^{\circ} \mathrm{C}$. After being stirred at $0{ }^{\circ} \mathrm{C}$ for $1.5 \mathrm{~h}$, the mixture was cooled to $-78{ }^{\circ} \mathrm{C}$ and quenched by the addition of $1 \mathrm{~mL}$ of $\mathrm{MeOH}$ followed by $30 \mathrm{~mL}$ of a saturated aqueous solution of potassium sodium tartrate. The mixture was then stirred at $23^{\circ} \mathrm{C}$ for $1 \mathrm{~h}$. The layers were separated, and the aqueous layer was extracted with $3 \times 30 \mathrm{~mL}$ of $\mathrm{CH}_{2} \mathrm{Cl}_{2}$. The combined organic layers were washed with brine $(20 \mathrm{~mL})$, dried $\left(\mathrm{MgSO}_{4}\right)$, and concentrated in vacuo. Purification by flash chromatography (15:85 to 20:80 EtOAc/hexanes) afforded 33 as a colorless oil $(0.900 \mathrm{~g}, 97 \%):{ }^{1} \mathrm{H}$ NMR $\left(\mathrm{CDCl}_{3}, 500 \mathrm{MHz}\right) \delta 7.34(\mathrm{~d}, J=8.5$ $\mathrm{Hz}, 2 \mathrm{H}), 6.93(\mathrm{~d}, J=8.7 \mathrm{~Hz}, 2 \mathrm{H}), 4.63(\mathrm{~d}, J=10.8 \mathrm{~Hz}, 1 \mathrm{H}), 4.56(\mathrm{~d}, J=10.8 \mathrm{~Hz}, 1 \mathrm{H}), 3.86(\mathrm{~s}, 3 \mathrm{H}), 3.65(\mathrm{~m}$, 2H), 3.29 (dd, $J=7.7,3.1 \mathrm{~Hz}, 1 \mathrm{H}), 2.01(\mathrm{~m}, 2 \mathrm{H}), 1.77(\mathrm{br}, 1 \mathrm{H}), 1.10(\mathrm{~d}, J=6.7 \mathrm{~Hz}, 3 \mathrm{H}), 0.98(\mathrm{~d}, J=7.0 \mathrm{~Hz}$, $3 \mathrm{H}), 0.97(\mathrm{~d}, J=6.8 \mathrm{~Hz}, 3 \mathrm{H}) ;{ }^{13} \mathrm{C} \mathrm{NMR}\left(\mathrm{CDCl}_{3}, 125 \mathrm{MHz}\right) 159.0,131.0,129.2,113.7,85.4,74.2,66.2,55.2$, 37.6, 30.8, 19.8, 19.4, 10.8; IR (thin film) $3417,1614,1514 \mathrm{~cm}^{-1} ;[\alpha]^{23}-12.9\left(c 1.20, \mathrm{CHCl}_{3}\right) ; \mathrm{HRMS}\left(\mathrm{CI} / \mathrm{NH}_{3}\right)$ $m / z$ calcd for $\mathrm{C}_{15} \mathrm{H}_{24} \mathrm{O}_{3}(\mathrm{M})^{+} 252.1725$, found 252.1721. Anal. Calcd for $\mathrm{C}_{15} \mathrm{H}_{24} \mathrm{O}_{3}: \mathrm{C}, 71.39 ; \mathrm{H}, 9.59$. Found: C, $71.10 ; \mathrm{H}, 9.62$.<smiles>CC(C)C(OC(C)(C)C)C(C)CO</smiles>

33

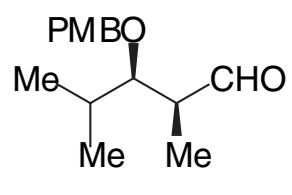

11

(2S,3R)-2,4-Dimethyl-3-(p-methoxybenzyloxy)pentanal (11). To a solution of oxalyl chloride ( $0.330 \mathrm{~mL}, 2.0$ $\mathrm{M}$ solution in $\left.\mathrm{CH}_{2} \mathrm{Cl}_{2}, 0.660 \mathrm{mmol}\right)$ in $4 \mathrm{~mL}$ of $\mathrm{CH}_{2} \mathrm{Cl}_{2}$ at $-78{ }^{\circ} \mathrm{C}$ was added a solution of DMSO $(0.094 \mathrm{~mL}$, $1.32 \mathrm{mmol}$ ) in $0.5 \mathrm{~mL}$ of $\mathrm{CH}_{2} \mathrm{Cl}_{2}$. The mixture was stirred at $-78^{\circ} \mathrm{C}$ for $10 \mathrm{~min}$, and then a solution of alcohol $33(0.151 \mathrm{~g}, 0.600 \mathrm{mmol})$ in $2 \mathrm{~mL}$ of $\mathrm{CH}_{2} \mathrm{Cl}_{2}$ was added dropwise by syringe. After $30 \mathrm{~min}$ at $-78{ }^{\circ} \mathrm{C}, 0.250$ $\mathrm{mL}(1.80 \mathrm{mmol})$ of $\mathrm{Et}_{3} \mathrm{~N}$ was added. The mixture was allowed to warm to $23{ }^{\circ} \mathrm{C}$ and was then poured into 10 $\mathrm{mL}$ of water. The layers were separated, and the aqueous layer was extracted with $3 \times 30 \mathrm{~mL}$ of $\mathrm{CH}_{2} \mathrm{Cl}_{2}$. The combined organic layers were washed with $1 \mathrm{~N} \mathrm{HCl}(10 \mathrm{~mL})$, aqueous $\mathrm{NaHCO}_{3}(10 \mathrm{~mL})$, brine $(10 \mathrm{~mL})$, dried $\left(\mathrm{MgSO}_{4}\right)$, and concentrated in vacuo. Purification by flash chromatography (5:95 EtOAc/hexanes) afforded 11 as a colorless oil $(0.140 \mathrm{~g}, 93 \%):{ }^{1} \mathrm{H}$ NMR $\left(\mathrm{CDCl}_{3}, 500 \mathrm{MHz}\right) \delta 9.84(\mathrm{~d}, J=1.0 \mathrm{~Hz}, 1 \mathrm{H}), 7.28(\mathrm{~d}, J=8.7 \mathrm{~Hz}$, 2H), $6.92(\mathrm{~d}, J=8.7 \mathrm{~Hz}, 2 \mathrm{H}), 4.46(\mathrm{~s}, 2 \mathrm{H}), 3.85(\mathrm{~s}, 3 \mathrm{H}), 3.64(\mathrm{dd}, J=7.4,3.5 \mathrm{~Hz}, 1 \mathrm{H}), 2.65(\mathrm{~m}, 1 \mathrm{H}), 1.96(\mathrm{~m}$, 
$1 \mathrm{H}), 1.22(\mathrm{~d}, J=7.0 \mathrm{~Hz}, 3 \mathrm{H}), 1.09(\mathrm{~d}, J=6.7 \mathrm{~Hz}, 3 \mathrm{H}), 1.00(\mathrm{~d}, J=6.8 \mathrm{~Hz}, 3 \mathrm{H}) ;{ }^{13} \mathrm{C} \mathrm{NMR}\left(\mathrm{CDCl}_{3}, 125 \mathrm{MHz}\right)$ 204.8, 159.1, 130.3, 129.2, 113.7, 83.2, 73.5, 55.2, 49.2, 31.2, 19.6, 18.9, 8.0; IR (thin film) 2961, 1721, 1613, $1514 \mathrm{~cm}^{-1} ;[\alpha]^{23}{ }_{\mathrm{D}}-41.4\left(c 1.45, \mathrm{CHCl}_{3}\right) ; \mathrm{HRMS}\left(\mathrm{CI} / \mathrm{NH}_{3}\right) \mathrm{m} / z$ calcd for $\mathrm{C}_{15} \mathrm{H}_{22} \mathrm{O}_{3}(\mathrm{M})^{+} 250.1569$, found 250.1564 .<smiles>CC(C)C(OS(C)(=O)=O)C(C)C</smiles>

11<smiles>C=C(C)C(OCc1ccccc1)C(C)(OBr)C(=O)CC</smiles>

10

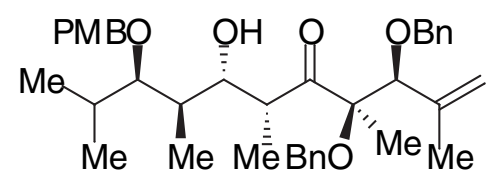

12

(3S,4R,6R,7S,8R,9R)-3,4-Dibenzyloxy-7-hydroxy-9-(p-methoxybenzyloxy)-2,4,6,8,10-pentamethylundec-1en-5-one (12). To a stirred suspension of $\mathrm{Sn}(\mathrm{OTf})_{2}(0.217 \mathrm{~g}, 0.520 \mathrm{mmol})$ in $2 \mathrm{~mL}$ of $\mathrm{CH}_{2} \mathrm{Cl}_{2}$ was added $\mathrm{Et}_{3} \mathrm{~N}$ $(0.089 \mathrm{~mL}, 0.640 \mathrm{mmol})$ at $23{ }^{\circ} \mathrm{C}$. The mixture was then cooled to $-78{ }^{\circ} \mathrm{C}$, and a solution of ketone $10(0.141$ $\mathrm{g}, 0.400 \mathrm{mmol})$ in $2 \mathrm{~mL}$ of $\mathrm{CH}_{2} \mathrm{Cl}_{2}$ was added. After $2 \mathrm{~h}$ at $-78{ }^{\circ} \mathrm{C}$, a solution of aldehyde $11(0.138 \mathrm{~g}, 0.550$ mmol) in $2 \mathrm{~mL}$ of $\mathrm{CH}_{2} \mathrm{Cl}_{2}$ was added, and the mixture was warmed to $-50{ }^{\circ} \mathrm{C}$. After $15 \mathrm{~h}, \mathrm{pH} 7$ buffer $(10 \mathrm{~mL})$ was added. The mixture was extracted with $3 \times 30 \mathrm{~mL}$ of $\mathrm{Et}_{2} \mathrm{O}$. The combined organic layers were washed with brine $(10 \mathrm{~mL})$, dried $\left(\mathrm{MgSO}_{4}\right)$, and concentrated in vacuo. Purification by flash chromatography (3:97 to 8:92 EtOAc/hexanes) afforded a colorless oil $\mathbf{1 2}$ as a single stereoisomer as determined by ${ }^{1} \mathrm{H}$ NMR spectroscopy of the crude mixture $(0.202 \mathrm{~g}, 84 \%):{ }^{1} \mathrm{H} \mathrm{NMR}\left(\mathrm{CDCl}_{3}, 500 \mathrm{MHz}\right) \delta 7.40(\mathrm{~m}, 2 \mathrm{H}), 7.30(\mathrm{~m}, 10 \mathrm{H})$, $6.82(\mathrm{~m}, 2 \mathrm{H}), 5.24(\mathrm{t}, J=1.6 \mathrm{~Hz}, 1 \mathrm{H}), 5.10(\mathrm{~s}, 1 \mathrm{H}), 4.75(\mathrm{~d}, J=11.6 \mathrm{~Hz}, 1 \mathrm{H}), 4.70(\mathrm{~d}, J=11.6 \mathrm{~Hz}, 1 \mathrm{H}), 4.48(\mathrm{~d}$, $J=11.5 \mathrm{~Hz}, 1 \mathrm{H}), 4.45(\mathrm{~d}, J=10.7 \mathrm{~Hz}, 1 \mathrm{H}), 4.39(\mathrm{~d}, J=10.7 \mathrm{~Hz}, 1 \mathrm{H}), 4.24(\mathrm{~d}, J=11.5 \mathrm{~Hz}, 1 \mathrm{H}), 4.20(\mathrm{dt}, J=$ $10.1,2.1 \mathrm{~Hz}, 1 \mathrm{H}), 4.18(\mathrm{~s}, 1 \mathrm{H}), 3.82(\mathrm{~s}, 3 \mathrm{H}), 3.50(\mathrm{~m}, 2 \mathrm{H}), 3.25(\mathrm{~d}, J=2.2 \mathrm{~Hz}, 1 \mathrm{H}), 1.92(\mathrm{~s}, 3 \mathrm{H}), 1.80(\mathrm{~m}, 2 \mathrm{H})$, $1.50(\mathrm{~s}, 3 \mathrm{H}), 1.09$ (d, $J=7.1 \mathrm{~Hz}, 3 \mathrm{H}), 1.07(\mathrm{~d}, J=7.2 \mathrm{~Hz}, 3 \mathrm{H}), 0.87(\mathrm{~d}, J=6.8 \mathrm{~Hz}, 3 \mathrm{H}), 0.71(\mathrm{~d}, J=7.0 \mathrm{~Hz}$, $3 \mathrm{H}) ;{ }^{13} \mathrm{C}$ NMR $\left(\mathrm{CDCl}_{3}, 125 \mathrm{MHz}\right) 220.2,158.8,141.3,138.3,137.1,131.8,129.3,128.33,128.29,127.8,127.3$, 126.9, 118.1, 113.5, 88.0, 87.8, 83.5, 74.6, 70.9, 70.2, 67.1, 55.2, 42.3, 37.2, 31.5, 20.5, 19.6, 19.4, 19.2, 8.9, 8.6; IR (thin film) $3519,1704,1613,1514 \mathrm{~cm}^{-1} ;[\alpha]^{23}{ }_{\mathrm{D}}+27.0\left(c 1.02, \mathrm{CHCl}_{3}\right)$; HRMS (ESI) $\mathrm{m} / \mathrm{z}$ calcd for $\mathrm{C}_{38} \mathrm{H}_{50} \mathrm{O}_{6} \mathrm{Na}(\mathrm{M}+\mathrm{Na})^{+}$625.3505, found 625.3517. Anal. Calcd for $\mathrm{C}_{38} \mathrm{H}_{50} \mathrm{O}_{6}: \mathrm{C}, 75.71 ; \mathrm{H}, 8.36$. Found: $\mathrm{C}$, 75.64; $\mathrm{H}, 8.45$. 
III. Stereochemical proofs of the model aldol product

A. The stereochemistry of $\mathrm{C} 7-\mathrm{OH}$ was determined by analysis of ${ }^{1} \mathrm{H}$ NMR coupling constant data and nOe data of acetal 34:<smiles>C=C(C)C(Cc1ccccc1)C(C)(O)C(=O)C(C)C(O)C(C)C(OC(C)(C)C)C(C)C</smiles>

12

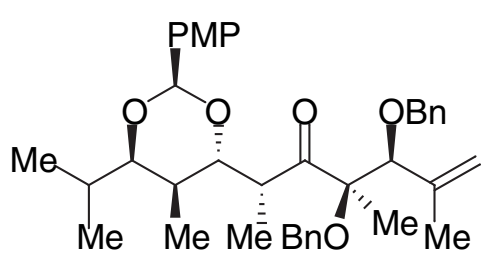

34

$(2 S, 4 S, 5 S, 6 R)-4-[(1 R, 3 R, 4 S)-3,4-D i b e n z y l o x y-2-o x 0-1,3,5$-trimethylhex-5-en-1-yl]-6-isopropyl-2-( $p$ methoxyphenyl)-5-methyl-1,3-dioxane (34). To a mixture of $\mathbf{1 2}(0.060 \mathrm{~g}, 0.100 \mathrm{mmol})$ and $0.080 \mathrm{~g}$ of activated $4 \AA$ molecular sieves powder in $1 \mathrm{~mL}$ of $\mathrm{CH}_{2} \mathrm{Cl}_{2}$ was added a solution of DDQ $(0.027 \mathrm{~g}, 0.120 \mathrm{mmol})$ in $2 \mathrm{~mL}$ of $\mathrm{CH}_{2} \mathrm{Cl}_{2}$ at $0{ }^{\circ} \mathrm{C}$ via cannula. After $1 \mathrm{~h}$, the mixture was filtered through Celite, and the filtrate was washed with $10 \mathrm{~mL}$ of aqueous saturated $\mathrm{NaHCO}_{3}$ solution. The aqueous layer was back-extracted with $2 \times 10$ $\mathrm{mL}$ of $\mathrm{CH}_{2} \mathrm{Cl}_{2}$. The combined organic layers were washed with brine $(10 \mathrm{~mL})$, dried $\left(\mathrm{MgSO}_{4}\right)$, and concentrated in vacuo. Purification by flash chromatography (1:99 EtOAc/benzene) afforded a white solid $\mathbf{3 4}$ as a single stereoisomer as determined by ${ }^{1} \mathrm{H}$ NMR spectroscopy of the crude mixture $(0.045 \mathrm{~g}, 75 \%)$ : $\mathrm{mp}$ 83-84 ${ }^{\circ} \mathrm{C} ;{ }^{1} \mathrm{H}$ NMR $\left(\mathrm{CDCl}_{3}, 500 \mathrm{MHz}\right) \delta$ 7.52-7.29 (m, $\left.12 \mathrm{H}\right), 6.94(\mathrm{~d}, J=8.6 \mathrm{~Hz}, 2 \mathrm{H}), 5.37(\mathrm{~s}, 1 \mathrm{H}), 5.31(\mathrm{~s}$, $1 \mathrm{H}), 5.12(\mathrm{~s}, 1 \mathrm{H}), 4.85(\mathrm{~d}, J=11.5 \mathrm{~Hz}, 1 \mathrm{H}), 4.80(\mathrm{~d}, J=11.5 \mathrm{~Hz}, 1 \mathrm{H}), 4.51(\mathrm{~d}, J=11.5 \mathrm{~Hz}, 1 \mathrm{H}), 4.36(\mathrm{~m}, 1 \mathrm{H})$, $4.32(\mathrm{~s}, 1 \mathrm{H}), 4.26(\mathrm{~d}, J=11.5 \mathrm{~Hz}, 1 \mathrm{H}), 4.10(\mathrm{~d}, J=11.1 \mathrm{~Hz}, 1 \mathrm{H}), 3.86(\mathrm{~s}, 3 \mathrm{H}), 3.30(\mathrm{dd}, J=9.8,1.8 \mathrm{~Hz}, 1 \mathrm{H})$, $1.99(\mathrm{~s}, 3 \mathrm{H}), 1.67(\mathrm{~m}, 1 \mathrm{H}), 1.61(\mathrm{~m}, 1 \mathrm{H}), 1.51(\mathrm{~s}, 3 \mathrm{H}), 1.28(\mathrm{~d}, J=6.8 \mathrm{~Hz}, 3 \mathrm{H}), 1.03(\mathrm{~d}, J=6.9 \mathrm{~Hz}, 3 \mathrm{H}), 0.82$ $(\mathrm{d}, J=6.4 \mathrm{~Hz}, 3 \mathrm{H}), 0.48(\mathrm{~d}, J=6.8 \mathrm{~Hz}, 3 \mathrm{H}) ;{ }^{13} \mathrm{C} \mathrm{NMR}\left(\mathrm{CDCl}_{3}, 125 \mathrm{MHz}\right) 217.3,159.7,141.6,138.4,137.6$, 132.0, 128.5, 128.2, 127.6, 127.4, 127.1, 118.3, 113.5, 95.2, 87.2, 87.0, 81.5, 81.1, 71.0, 67.4, 55.3, 38.2, 31.1, 29.1, 21.0, 19.4, 19.2, 16.8, 16.2, 13.2; IR (KBr) 1708, 1617, $1518 \mathrm{~cm}^{-1}$; $[\alpha]^{23}{ }_{\mathrm{D}}-4.4\left(c\right.$ 1.25, $\left.\mathrm{CHCl}_{3}\right)$; HRMS (ESI) $m / z$ calcd for $\mathrm{C}_{38} \mathrm{H}_{48} \mathrm{O}_{6} \mathrm{Na}(\mathrm{M}+\mathrm{Na})^{+} 623.3348$, found 623.3358. Anal. Calcd for $\mathrm{C}_{38} \mathrm{H}_{48} \mathrm{O}_{6}: \mathrm{C}, 75.97 ; \mathrm{H}$, 8.05. Found: $\mathrm{C}, 76.17 ; \mathrm{H}, 8.05$.

Relevant ${ }^{1} \mathrm{H}$ NMR coupling constants and DPFGSE-nOe data (mixing time 0.5s): (the peaks in the ${ }^{1} \mathrm{H}$ NMR spectra were assigned using ${ }^{1} \mathrm{H} /{ }^{1} \mathrm{H} \mathrm{COSY},{ }^{1} \mathrm{H} /{ }^{13} \mathrm{C} \mathrm{HMQC},{ }^{1} \mathrm{H}$ NMR chemical shifts, and ${ }^{1} \mathrm{H}$ NMR coupling constants)

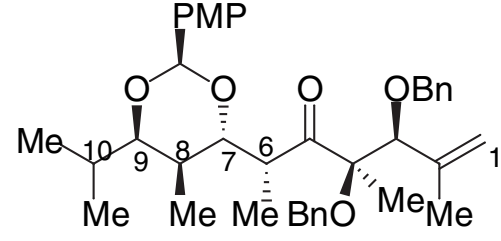

34

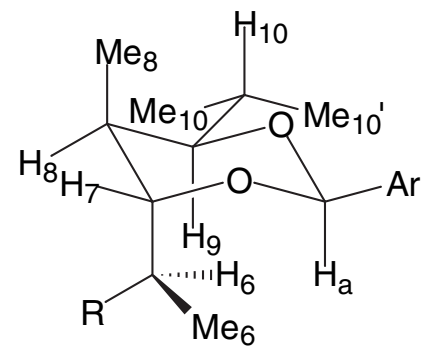
${ }^{1} \mathrm{H}$ NMR coupling constants:
$\mathbf{J}_{89}=1.8 \mathrm{~Hz}$
nOe data:
$\mathrm{J}_{78}=0 \mathrm{~Hz}$
$\mathbf{H}_{\mathbf{a}}$ irradiated: $\mathrm{H}_{6}(5.1 \%), \mathrm{H}_{9}(5.4 \%), \mathrm{Me}_{6}(2.1 \%)$
$\mathbf{H}_{9}$ irradiated: $\mathrm{H}_{\mathrm{a}}(6.8 \%), \mathrm{H}_{6}(6.6 \%), \mathrm{H}_{8}(4.2 \%), \mathrm{Me}_{10}(2.2 \%), \mathrm{Me}_{10^{\prime}}(2.9 \%)$
$\mathbf{H}_{7}$ irradiated: $\mathrm{H}_{8}(2.3 \%), \mathrm{Me}_{6}(1.9 \%), \mathrm{Me}_{8}(3.0 \%)$
$\mathbf{H}_{8}$ irradiated: $\mathrm{H}_{6}(1.3 \%), \mathrm{H}_{7}(2.0 \%), \mathrm{H}_{9}(2.3 \%), \mathrm{Me}_{8}(2.4 \%), \mathrm{Me}_{10}(2.4 \%)$ 
Note: The absence of nOe between $\mathrm{H}_{7}$ and $\mathrm{H}_{9}$ indicates that $\mathrm{C} 7$ and $\mathrm{C} 9$ are anti, and the absence of nOe between $\mathrm{H}_{a}$ and $\mathrm{H}_{7}$ and the observed nOe between $\mathrm{H}_{a}$ and $\mathrm{H}_{9}$ indicate that the acetal proton $\mathrm{H}_{a}$ and $\mathrm{H}_{9}$ take axial positions and $\mathrm{H}_{7}$ is equatorial.

B. The stereochemistry of C8-Me was determined by analysis of ${ }^{1} \mathrm{H}$ NMR coupling constant data and nOe data of acetonide 35:

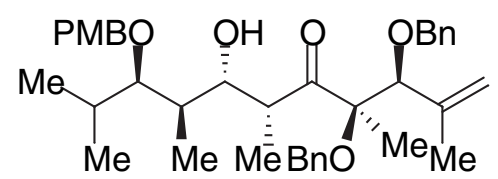

12

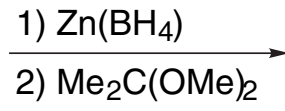

2) $\mathrm{Me}_{2} \mathrm{C}(\mathrm{OMe})_{2}$<smiles>C=C(Cc1ccccc1)C(O)C(O)(O)C(O)C(O)C(C)C(C)C(O)C(C)C</smiles>

36

$(3 S, 4 R, 5 S, 6 R, 7 S, 8 R, 9 R)-3,4-D i b e n z y l o x y-9-(p-m e t h o x y b e n z y l o x y)-2,4,6,8,10$-pentamethylundec-1-en-5,7diol (36). To a cooled solution $\left(-40^{\circ} \mathrm{C}\right)$ of ketone $12(0.060 \mathrm{~g}, 0.10 \mathrm{mmol})$ in $1 \mathrm{~mL}$ of $\mathrm{Et}_{2} \mathrm{O}$ was added $1.5 \mathrm{~mL}$ $\left(0.30 \mathrm{mmol}, 0.20 \mathrm{M}\right.$ in $\left.\mathrm{Et}_{2} \mathrm{O}\right)$ of $\mathrm{Zn}\left(\mathrm{BH}_{4}\right)_{2}$. After $12 \mathrm{~h}$, the excess hydride was quenched by $2 \mathrm{~mL}$ each of aqueous saturated $\mathrm{NH}_{4} \mathrm{Cl}$ solution and $1 \mathrm{M} \mathrm{HCl}$. The mixture was extracted with $3 \times 10 \mathrm{~mL} \mathrm{Et}_{2} \mathrm{O}$, and the combined organic extracts were dried over $\mathrm{Mg}_{2} \mathrm{SO}_{4}$, filtered, and concentrated in vacuo. Purification by flash chromatography (10:90 to 20:80 $\mathrm{Et}_{2} \mathrm{O} /$ hexanes) afforded a colorless oil $\mathbf{3 6}$ as a single stereoisomer $(0.032 \mathrm{~g}$, 53\%): ${ }^{1} \mathrm{H}$ NMR $\left(\mathrm{CDCl}_{3}, 500 \mathrm{MHz}\right) \delta 7.43-7.28(\mathrm{~m}, 12 \mathrm{H}), 6.90(\mathrm{~m}, 2 \mathrm{H}), 5.26(\mathrm{~s}, 1 \mathrm{H}), 5.12(\mathrm{~s}, 1 \mathrm{H}), 4.74(\mathrm{~d}, J=$ $11.5 \mathrm{~Hz}, 1 \mathrm{H}), 4.68(\mathrm{~d}, J=11.0 \mathrm{~Hz}, 1 \mathrm{H}), 4.66(\mathrm{~d}, J=11.5 \mathrm{~Hz}, 1 \mathrm{H}), 4.62(\mathrm{~d}, J=11.4 \mathrm{~Hz}, 1 \mathrm{H}), 4.60(\mathrm{~d}, J=11.0$ $\mathrm{Hz}, 1 \mathrm{H}), 4.33(\mathrm{~d}, J=11.4 \mathrm{~Hz}, 1 \mathrm{H}), 4.14(\mathrm{~s}, 1 \mathrm{H}), 4.07(\mathrm{dd}, J=4.9,1.4 \mathrm{~Hz}, 1 \mathrm{H}), 3.84(\mathrm{~s}, 3 \mathrm{H}), 3.76(\mathrm{~d}, J=9.8 \mathrm{~Hz}$, $1 \mathrm{H}), 3.59$ (br, 1H), 3.57 (d, $J=4.9 \mathrm{~Hz}, 1 \mathrm{H}), 3.51(\mathrm{dd}, J=9.0,1.6 \mathrm{~Hz}, 1 \mathrm{H}), 2.17(\mathrm{~m}, 1 \mathrm{H}), 1.92(\mathrm{~s}, 3 \mathrm{H}), 1.86(\mathrm{~m}$, $2 \mathrm{H}), 1.45(\mathrm{~s}, 3 \mathrm{H}), 1.10(\mathrm{~d}, J=6.6 \mathrm{~Hz}, 3 \mathrm{H}), 1.03(\mathrm{~d}, J=6.9 \mathrm{~Hz}, 3 \mathrm{H}), 0.92(\mathrm{~d}, J=6.8 \mathrm{~Hz}, 3 \mathrm{H}), 0.82(\mathrm{~d}, J=6.9$ $\mathrm{Hz}, 3 \mathrm{H}) ;{ }^{13} \mathrm{C} \mathrm{NMR}\left(\mathrm{CDCl}_{3}, 125 \mathrm{MHz}\right) 158.9,141.7,139.2,137.7,131.8,129.2,128.5,128.2,127.9,127.8$, 127.1, 127.0, 117.5, 113.6, 88.5, 84.5, 80.9, 79.0, 78.5, 74.4, 70.9, 65.1, 55.2, 37.5, 34.1, 31.2, 20.4, 19.8, 18.2, 9.8, 6.2; IR (thin film) 3488, 1613, $1514 \mathrm{~cm}^{-1} ;[\alpha]_{\mathrm{D}}^{23}+14.2\left(c 0.60, \mathrm{CHCl}_{3}\right)$; HRMS (ESI) $\mathrm{m} / \mathrm{z}$ calcd for $\mathrm{C}_{38} \mathrm{H}_{52} \mathrm{O}_{6} \mathrm{Na}(\mathrm{M}+\mathrm{Na})^{+}$627.3661, found 627.3670 .<smiles>C=C(C)C(Cc1ccccc1)C(O)C(O)C(O)C(O)C(C)C(OC(C)(C)C)C(C)C</smiles>

36<smiles>C=C(C)C(Cc1ccccc1)C(OC)(OC)C1OC(C)(C)OC(C(C)C(OC(C)(C)C)C(O)C(C)C)C1C(C)C</smiles>

35

$(4 S, 5 R, 6 S)-4-[(1 R, 2 S)-1,2-D i b e n z y l o x y-1,3-d i m e t h y l-b u t-3-e n y l]-6-[(1 S, 2 R)-1,3-d i m e t h y l-2-(p-$ methoxybenzyloxy)butyl]-2,2,5-trimethyl-1,3-dioxane (35). To a solution of diol 36 (0.025 g, $0.041 \mathrm{mmol})$ 
in $1 \mathrm{~mL}$ of 2,2-dimethoxypropane at $23{ }^{\circ} \mathrm{C}$ was added CSA $(0.0010 \mathrm{~g}, 0.0040 \mathrm{mmol})$. The mixture was stirred for $12 \mathrm{~h}$ before $0.05 \mathrm{~mL}$ of $\mathrm{Et}_{3} \mathrm{~N}$ was added. The mixture was then concentrated in vacuo. Purification by flash chromatography $\left(5: 95 \mathrm{Et}_{2} \mathrm{O} /\right.$ hexanes $)$ afforded 35 as a colorless oil $(0.020 \mathrm{~g}, 76 \%):{ }^{1} \mathrm{H} \mathrm{NMR}\left(\mathrm{CDCl}_{3}, 500 \mathrm{MHz}\right)$ $\delta 7.42-7.28(\mathrm{~m}, 12 \mathrm{H}), 6.93(\mathrm{~m}, 2 \mathrm{H}), 5.15(\mathrm{t}, J=1.6 \mathrm{~Hz}, 1 \mathrm{H}), 5.07(\mathrm{~s}, 1 \mathrm{H}), 4.90(\mathrm{~d}, J=11.9 \mathrm{~Hz}, 1 \mathrm{H}), 4.82(\mathrm{~d}, J$ $=11.9 \mathrm{~Hz}, 1 \mathrm{H}), 4.60(\mathrm{~d}, J=11.0 \mathrm{~Hz}, 1 \mathrm{H}), 4.58(\mathrm{~d}, J=11.7 \mathrm{~Hz}, 1 \mathrm{H}), 4.56(\mathrm{~d}, J=11.0 \mathrm{~Hz}, 1 \mathrm{H}), 4.30(\mathrm{~d}, J=11.7$ $\mathrm{Hz}, 1 \mathrm{H}), 4.09(\mathrm{~d}, J=1.9 \mathrm{~Hz}, 1 \mathrm{H}), 3.94(\mathrm{~s}, 1 \mathrm{H}), 3.87(\mathrm{~s}, 3 \mathrm{H}), 3.81(\mathrm{dd}, J=9.9,1.7 \mathrm{~Hz}, 1 \mathrm{H}), 3.43(\mathrm{dd}, J=8.9,1.2$ $\mathrm{Hz}, 1 \mathrm{H}), 2.01(\mathrm{~m}, 1 \mathrm{H}), 1.93(\mathrm{~m}, 1 \mathrm{H}), 1.89$ (s, 3H), $1.84(\mathrm{~m}, 1 \mathrm{H}), 1.48(\mathrm{~s}, 3 \mathrm{H}), 1.47(\mathrm{~s}, 3 \mathrm{H}), 1.34(\mathrm{~s}, 3 \mathrm{H}), 1.10(\mathrm{~d}$, $J=6.5 \mathrm{~Hz}, 3 \mathrm{H}), 1.08(\mathrm{~d}, J=6.6 \mathrm{~Hz}, 3 \mathrm{H}), 0.93(\mathrm{~d}, J=6.8 \mathrm{~Hz}, 3 \mathrm{H}), 0.79(\mathrm{~d}, J=7.0 \mathrm{~Hz}, 3 \mathrm{H}) ;{ }^{13} \mathrm{C} \mathrm{NMR}\left(\mathrm{CDCl}_{3}\right.$, $125 \mathrm{MHz}) 158.9,142.4,140.3,138.8,131.8,128.5,128.2,128.1,127.6,127.2,127.1,126.8,118.1,113.7,99.2$, 90.5, 83.3, 80.7, 75.9, 75.3, 74.2, 70.1, 66.6, 55.3, 36.5, 31.6, 31.2, 30.1, 20.4, 19.8, 19.6, 18.5, 16.2, 7.9, 6.9; IR (thin film) 2916, 1613, $1514 \mathrm{~cm}^{-1} ;[\alpha]^{23}+19.5\left(c 0.95, \mathrm{CHCl}_{3}\right) ; \mathrm{HRMS}$ (ESI) $m / z$ calcd for $\mathrm{C}_{41} \mathrm{H}_{56} \mathrm{O}_{6} \mathrm{Na}_{(\mathrm{M}}$ $+\mathrm{Na})^{+}$667.3975, found 667.3980.

Relevant ${ }^{1} \mathrm{H}$ NMR coupling constants, ${ }^{13} \mathrm{C}$ chemical shifts, and DPFGSE-nOe data (mixing time 0.5s): (the peaks in the ${ }^{1} \mathrm{H}$ NMR and ${ }^{13} \mathrm{C}$ NMR spectra were assigned using ${ }^{1} \mathrm{H} /{ }^{1} \mathrm{H} \mathrm{COSY},{ }^{1} \mathrm{H} /{ }^{13} \mathrm{C} \mathrm{HMQC}$, ${ }^{1} \mathrm{H} \mathrm{NMR}$ chemical shifts, ${ }^{13} \mathrm{C}$ NMR chemical shifts, and ${ }^{1} \mathrm{H}$ NMR coupling constants)<smiles>C=C(C)C(Cc1ccccc1)C(OC)C(OC(C)(C)C)C(OC(C)(C)C)C(C)C(OC(C)(C)C)C(C)C</smiles>

35

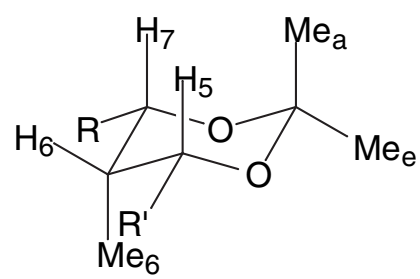

\begin{tabular}{|l|l|l|}
\hline${ }^{13} \mathbf{C}$ NMR chemical shits: & ${ }^{1} \mathbf{H}$ NMR coupling constants: & nOe data: \\
$\mathbf{M e}_{\mathrm{a}}: 19.8 \mathrm{ppm}$ & $\mathbf{H}_{\mathbf{5}}$ irradiated: $\mathrm{H}_{7}(6.4 \%), \mathrm{H}_{6}(4.4 \%), \mathrm{Me}_{\mathrm{a}}(5.6 \%)$ \\
$\mathbf{M e}_{\mathrm{e}}: 30.1 \mathrm{ppm}$ & $\mathrm{J}_{56}=1.7 \mathrm{~Hz}$ & $\mathbf{H}_{\mathbf{6}}$ irradiated: $\mathrm{H}_{5}(4.2 \%), \mathrm{H}_{7}(3.9 \%), \mathrm{Me}_{6}(4.0 \%)$ \\
& $\mathrm{J}_{67}=1.7 \mathrm{~Hz}$ & $\mathbf{H}_{7}$ irradiated: $\mathrm{H}_{5}(6.1 \%), \mathrm{H}_{6}(4.7 \%), \mathrm{Me}_{\mathrm{a}}(5.1 \%)$ \\
\hline
\end{tabular}

Note: The ${ }^{13} \mathrm{C}$ NMR chemical shifts of the ketal carbons indicate the syn $\mathrm{C} 5 / \mathrm{C} 7$ relationship and the chair conformation of the acetonide. ${ }^{4}$

\footnotetext{
${ }^{4}$ Rychnovsky, S. D.; Rogers, B.; Yang, G. J. Org. Chem. 1993, 58, 3511-3515.
} 


\section{Total synthesis of (9S)-dihydroerythronolide A}

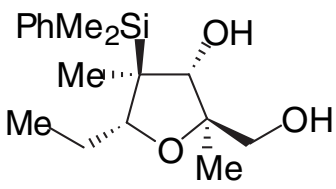

$(+)-13$

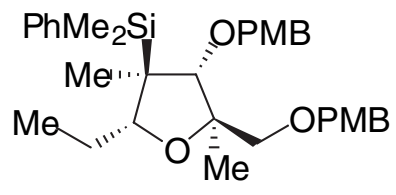

37

$(2 R, 3 R, 4 R, 5 R)-2,4-D i m e t h y l-4-d i m e t h y l p h e n y l s i l y l-5-e t h y l-3-(p-m e t h o x y b e n z y l o x y)-2-(p-$

methoxybenzyloxymethyl)tetrahydrofuran (37). To a suspension of $\mathrm{NaH}(1.74 \mathrm{~g}, 60 \%$ oil dispersion, 43.6 mmol, washed with $3 \times 20 \mathrm{~mL}$ of hexanes) in $30 \mathrm{~mL}$ of dry DMF was added a solution of diol (+)-13 (3.35 g, $10.9 \mathrm{mmol})$ in $30 \mathrm{~mL}$ of DMF. After $0.5 \mathrm{~h}$ at $23{ }^{\circ} \mathrm{C}, p$-methoxybenzyl chloride $(5.91 \mathrm{~mL}, 43.6 \mathrm{mmol})$ and $n$ $\mathrm{Bu}_{4} \mathrm{NI}(0.200 \mathrm{~g}, 0.545 \mathrm{mmol})$ were added, and the reaction mixture was stirred for $12 \mathrm{~h}$ at $23{ }^{\circ} \mathrm{C}$. To the

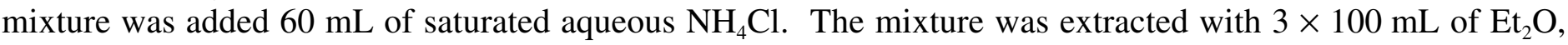
and the combined organic layers were washed with brine $(40 \mathrm{~mL})$, dried $\left(\mathrm{MgSO}_{4}\right)$, and concentrated in vacuo. Purification by flash chromatography (5:95 EtOAc/hexanes) afforded 37 as a colorless oil $(5.75 \mathrm{~g}, 96 \%):{ }^{1} \mathrm{H}$ $\operatorname{NMR}\left(\mathrm{CDCl}_{3}, 500 \mathrm{MHz}\right) \delta 7.58(\mathrm{~m}, 2 \mathrm{H}), 7.45(\mathrm{~m}, 3 \mathrm{H}), 7.34(\mathrm{~d}, J=8.6 \mathrm{~Hz}, 2 \mathrm{H}), 7.23(\mathrm{~d}, J=8.6 \mathrm{~Hz}, 2 \mathrm{H}), 6.99$ $(\mathrm{d}, J=8.6 \mathrm{~Hz}, 2 \mathrm{H}), 6.94(\mathrm{~d}, J=8.7 \mathrm{~Hz}, 2 \mathrm{H}), 4.59(\mathrm{~d}, J=11.0 \mathrm{~Hz}, 1 \mathrm{H}), 4.54(\mathrm{~d}, J=11.7 \mathrm{~Hz}, 1 \mathrm{H}), 4.48(\mathrm{~d}, J=$ $11.7 \mathrm{~Hz}, 1 \mathrm{H}), 4.22(\mathrm{~d}, J=11.0 \mathrm{~Hz}, 1 \mathrm{H}), 4.05(\mathrm{~s}, 1 \mathrm{H}), 3.91(\mathrm{~s}, 3 \mathrm{H}), 3.89(\mathrm{~s}, 3 \mathrm{H}), 3.62(\mathrm{~d}, J=9.9 \mathrm{~Hz}, 1 \mathrm{H}), 3.30$ $(\mathrm{d}, J=9.2 \mathrm{~Hz}, 1 \mathrm{H}), 3.20(\mathrm{~d}, J=9.2 \mathrm{~Hz}, 1 \mathrm{H}), 1.48(\mathrm{~m}, 1 \mathrm{H}), 1.34(\mathrm{~m}, 1 \mathrm{H}$ and s, 3H), $1.15(\mathrm{~s}, 3 \mathrm{H}), 1.03(\mathrm{t}, J=7.3$ $\mathrm{Hz}, 3 \mathrm{H}), 0.41(\mathrm{~s}, 3 \mathrm{H}), 0.40(\mathrm{~s}, 3 \mathrm{H}) ;{ }^{13} \mathrm{C} \mathrm{NMR}\left(\mathrm{CDCl}_{3}, 125 \mathrm{MHz}\right) \delta 159.0,158.8,137.1,134.3,131.0,130.6$, 129.1, 129.0, 128.8, 127.6, 113.6, 113.5, 86.0, 81.9, 81.4, 75.8, 73.0, 71.7, 55.20, 55.16, 37.4, 23.9, 20.8, 12.2, 9.7, -4.8, -5.1; IR (thin film) 2959, 1613, 1513, 1247, $1082 \mathrm{~cm}^{-1} ;[\alpha]^{23}{ }_{\mathrm{D}}-13.7\left(c 1.17, \mathrm{CHCl}_{3}\right)$; HRMS (ESI) $\mathrm{m} /$ $z$ calcd for $\mathrm{C}_{33} \mathrm{H}_{43} \mathrm{O}_{5} \mathrm{Si}(\mathrm{M}-\mathrm{H})^{+}$547.2880, found 547.2869. Anal. Calcd for $\mathrm{C}_{33} \mathrm{H}_{44} \mathrm{O}_{5} \mathrm{Si}: \mathrm{C}, 72.22 ; \mathrm{H}, 8.08$. Found: C, 72.33; H, 8.23.

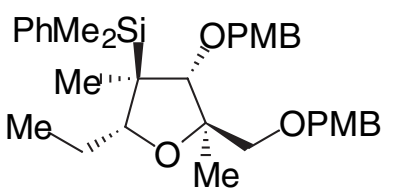

37

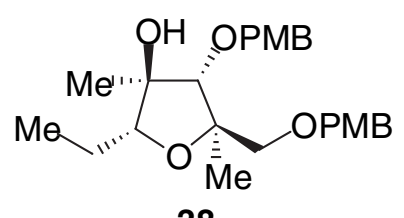

38

$(2 R, 3 R, 4 R, 5 R)-2,4-D i m e t h y l-5-e t h y l-4-h y d r o x y l-3-(p-m e t h o x y b e n z y l o x y)-2-(p-$

methoxybenzyloxymethyl)tetrahydrofuran (38). To a suspension of $\mathrm{KH}(6.28 \mathrm{~g}, 157 \mathrm{mmol}) \mathrm{in} 50 \mathrm{~mL}$ of NMP was added cumene hydroperoxide $(21.2 \mathrm{~mL}, 88.0 \%, 126 \mathrm{mmol})$ dropwise at $0{ }^{\circ} \mathrm{C}$. After $20 \mathrm{~min}$ at $0{ }^{\circ} \mathrm{C}$, silane $37(8.60 \mathrm{~g}, 15.7 \mathrm{mmol})$ in $50 \mathrm{~mL}$ of NMP was added dropwise followed by $n$-Bu $\mathrm{Br}_{4}(31.4 \mathrm{~mL}, 1 \mathrm{M}$ solution in THF, $31.4 \mathrm{mmol}$ ). The mixture was allowed to stir at $60{ }^{\circ} \mathrm{C}$ for $15 \mathrm{~h}$, at which point $100 \mathrm{~mL}$ of saturated aqueous $\mathrm{Na}_{2} \mathrm{~S}_{2} \mathrm{O}_{3}$ solution was added. The mixture was stirred for 30 min at $23{ }^{\circ} \mathrm{C}$, and was then extracted with $5 \times 100 \mathrm{~mL}$ of $t$-BuOMe. The combined organic layers were washed with brine $(50 \mathrm{~mL})$, dried $\left(\mathrm{MgSO}_{4}\right)$, and concentrated in vacuo. The residue was then subjected to bulb-to-bulb distillation $\left(70{ }^{\circ} \mathrm{C} / 0.1\right.$ $\mathrm{mmHg}$ ) to remove most of the NMP and cumene alcohol. Further purification by flash chromatography (5:95 to $25: 75 \mathrm{EtOAc/hexanes)}$ afforded $38(5.74 \mathrm{~g}, 85 \%)$ as colorless oil: ${ }^{1} \mathrm{H} \mathrm{NMR}\left(\mathrm{CDCl}_{3}, 500 \mathrm{MHz}\right) \delta 7.33(\mathrm{~d}, J=$ $8.6 \mathrm{~Hz}, 2 \mathrm{H}), 7.28(\mathrm{~d}, J=8.6 \mathrm{~Hz}, 2 \mathrm{H}), 6.93(\mathrm{~m}, 4 \mathrm{H}), 4.63(\mathrm{~d}, J=11.6 \mathrm{~Hz}, 1 \mathrm{H}), 4.60(\mathrm{~d}, J=11.6 \mathrm{~Hz}, 1 \mathrm{H}), 4.56$ $(\mathrm{d}, J=9.6 \mathrm{~Hz}, 1 \mathrm{H}), 4.52(\mathrm{~d}, J=9.6 \mathrm{~Hz}, 1 \mathrm{H}), 3.87(\mathrm{~s}, 1 \mathrm{H}), 3.86(\mathrm{~s}, 3 \mathrm{H}), 3.85(\mathrm{~s}, 3 \mathrm{H}), 3.66(\mathrm{dd}, J=9.5,4.5 \mathrm{~Hz}$, $1 \mathrm{H}), 3.47(\mathrm{~d}, J=9.7 \mathrm{~Hz}, 1 \mathrm{H}), 3.37(\mathrm{~d}, J=9.7 \mathrm{~Hz}, 1 \mathrm{H}), 3.19(\mathrm{~s}, 1 \mathrm{H}), 1.54(\mathrm{~m}, 2 \mathrm{H}), 1.31(\mathrm{~s}, 3 \mathrm{H}), 1.22(\mathrm{~s}, 3 \mathrm{H})$, 
$1.04(\mathrm{t}, J=7.4 \mathrm{~Hz}, 3 \mathrm{H}) ;{ }^{13} \mathrm{C}$ NMR $\left(\mathrm{CDCl}_{3}, 125 \mathrm{MHz}\right) \delta 159.2,159.1,130.6,129.8,129.3,129.1,113.7,113.6$, 88.6, 85.8, 82.2, 81.0, 76.2, 73.2, 73.1, 55.1, 23.1, 20.3, 16.8, 10.7; IR (thin film) 3455, 1613, 1513, 1247, 1084 $\mathrm{cm}^{-1} ;[\alpha]_{D}^{23}+44.4\left(c\right.$ 1.08, $\left.\mathrm{CHCl}_{3}\right)$; HRMS $\left(\mathrm{CI} / \mathrm{NH}_{3}\right) \mathrm{m} / \mathrm{z}$ calcd for $\mathrm{C}_{25} \mathrm{H}_{33} \mathrm{O}_{6}(\mathrm{M}-\mathrm{H})^{+}$429.2277, found 429.2278. Anal. Calcd for $\mathrm{C}_{25} \mathrm{H}_{34} \mathrm{O}_{6}$ : C, 69.74; H, 7.96. Found: $\mathrm{C}, 69.47 ; \mathrm{H}, 8.12$.

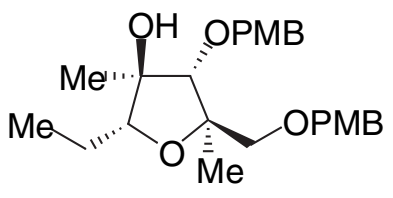

38

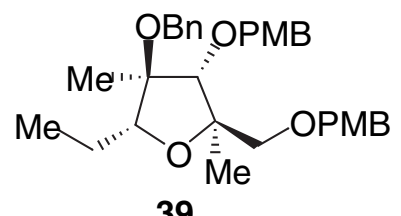

39

$(2 R, 3 R, 4 R, 5 R)-4-B e n z y l o x y-2,4-d i m e t h y l-5-e t h y l-3-(p-m e t h o x y b e n z y l o x y)-2-(p-$

methoxybenzyloxymethyl)tetrahydrofuran (39). To a suspension of $\mathrm{NaH}(3.12 \mathrm{~g}, 60 \%$ oil dispersion, 78.0 $\mathrm{mmol}$, washed with $3 \times 30 \mathrm{~mL}$ of hexanes) in $100 \mathrm{~mL}$ of dry DMF was added a solution of diol $\mathbf{3 8}(11.2 \mathrm{~g}, 26.0$ $\mathrm{mmol})$ in $100 \mathrm{~mL}$ of DMF at $0{ }^{\circ} \mathrm{C}$. After $0.5 \mathrm{~h}$ at $0{ }^{\circ} \mathrm{C}, \mathrm{BnBr}(9.30 \mathrm{~mL}, 78.0 \mathrm{mmol})$ and $n-\mathrm{Bu}_{4} \mathrm{NI}(0.480 \mathrm{~g}, 1.30$ $\mathrm{mmol}$ ) were added, and the reaction mixture was stirred for $12 \mathrm{~h}$ at $23{ }^{\circ} \mathrm{C}$. A saturated aqueous solution of $\mathrm{NH}_{4} \mathrm{Cl}(100 \mathrm{~mL})$ was then added. The mixture was extracted with $3 \times 200 \mathrm{~mL}$ of $\mathrm{Et}_{2} \mathrm{O}$, and the combined organic layers were washed with brine $(50 \mathrm{~mL})$, dried $\left(\mathrm{MgSO}_{4}\right)$, and concentrated in vacuo. Purification by flash chromatography (5:95 to 10:90 EtOAc/hexanes) afforded 39 as a colorless oil (12.8 g, 95\%): ${ }^{1} \mathrm{H}$ NMR $\left(\mathrm{CDCl}_{3}, 500 \mathrm{MHz}\right) \delta 7.36(\mathrm{~m}, 9 \mathrm{H}), 6.96(\mathrm{~m}, 4 \mathrm{H}), 4.65(\mathrm{~m}, 4 \mathrm{H}), 4.48(\mathrm{~d}, J=11.5 \mathrm{~Hz}, 1 \mathrm{H}), 4.43(\mathrm{~d}, J=11.7 \mathrm{~Hz}$, $1 \mathrm{H}), 4.23(\mathrm{~s}, 1 \mathrm{H}), 3.89$ (s, $3 \mathrm{H}), 3.88(\mathrm{~s}, 3 \mathrm{H}), 3.81(\mathrm{dd}, J=8.7,4.1 \mathrm{~Hz}, 1 \mathrm{H}), 3.57(\mathrm{~d}, J=9.3 \mathrm{~Hz}, 1 \mathrm{H}), 3.50(\mathrm{~d}, J=$ $9.3 \mathrm{~Hz}, 1 \mathrm{H}), 1.67(\mathrm{~m}, 1 \mathrm{H}), 1.60(\mathrm{~m}, 1 \mathrm{H}), 1.41(\mathrm{~s}, 3 \mathrm{H}), 1.34(\mathrm{~s}, 3 \mathrm{H}), 1.10(\mathrm{t}, J=7.4 \mathrm{~Hz}, 3 \mathrm{H}) ;{ }^{13} \mathrm{C}$ NMR $\left(\mathrm{CDCl}_{3}\right.$, $125 \mathrm{MHz}) \delta 159.08,159.05,139.4,130.6,130.4,129.3,129.2,128.1,127.1,127.0,113.68,113.63,87.1,84.5$, 81.7, 81.1, 76.1, 73.1, 71.6, 65.4, 55.2, 22.6, 20.1, 13.10, 10.9; IR (thin film) 2934, 1613, 1513, 1247, $1086 \mathrm{~cm}$ ${ }^{1} ;[\alpha]^{23}{ }_{D}-35.1\left(c 1.14, \mathrm{CHCl}_{3}\right)$; HRMS $\left(\mathrm{CI} / \mathrm{NH}_{3}\right) \mathrm{m} / \mathrm{z}$ calcd for $\mathrm{C}_{32} \mathrm{H}_{39} \mathrm{O}_{6}(\mathrm{M}-\mathrm{H})^{+}$519.2747, found 519.2742. Anal. Calcd for $\mathrm{C}_{32} \mathrm{H}_{40} \mathrm{O}_{6}$ : C, 73.82; H, 7.74. Found: C, 73.79; H, 7.90.

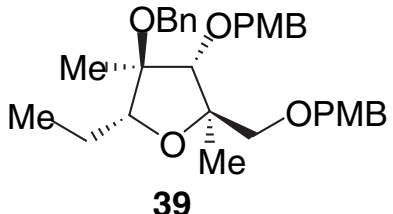

39

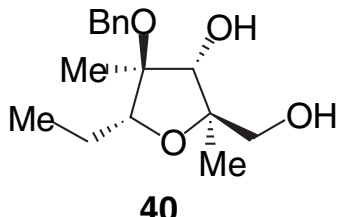

40

(2R,3R,4R,5R)-4-Benzyloxy-2,4-dimethyl-5-ethyl-3-hydroxy-2-hydroxymethyltetrahydrofuran (40). To a solution of 39 (12.5 g, $24.0 \mathrm{mmol})$ in $200 \mathrm{~mL}$ of $\mathrm{CH}_{3} \mathrm{CN}$ and $20 \mathrm{~mL}$ of $\mathrm{H}_{2} \mathrm{O}$ was added ceric ammonium nitrate $(65.8 \mathrm{~g}, 120 \mathrm{mmol})$. After $1 \mathrm{~h}$ at $23{ }^{\circ} \mathrm{C}, 300 \mathrm{~mL}$ of saturated aqueous $\mathrm{NaHCO}_{3}$ solution and $20.0 \mathrm{~g}$ of $\mathrm{NaHSO}_{3}$ were added to the reaction mixture. After $15 \mathrm{~min}$, the mixture was extracted with $4 \times 500 \mathrm{~mL}$ of EtOAc. The combined organic layers were washed with brine $(100 \mathrm{~mL})$, dried $\left(\mathrm{MgSO}_{4}\right)$, and concentrated in vacuo. Purification by flash chromatography (30:70 to 50:50 EtOAc/hexanes) afforded $\mathbf{4 0}$ as a white solid (6.45 g, 96\%): $\mathrm{mp} 82-82{ }^{\circ} \mathrm{C} ;{ }^{1} \mathrm{H}$ NMR $\left(\mathrm{CDCl}_{3}, 500 \mathrm{MHz}\right) \delta 7.35(\mathrm{~m}, 5 \mathrm{H}), 4.70(\mathrm{~d}, J=11.6 \mathrm{~Hz}, 1 \mathrm{H}), 4.67$ (d, $J=11.6$ $\mathrm{Hz}, 1 \mathrm{H}), 4.27(\mathrm{~d}, J=6.1 \mathrm{~Hz}, 1 \mathrm{H}), 3.74(\mathrm{dd}, J=9.0,4.2 \mathrm{~Hz}, 1 \mathrm{H}), 3.61(\mathrm{dd}, J=10.9,2.4 \mathrm{~Hz}, 1 \mathrm{H}), 3.51(\mathrm{dd}, J=$ $10.9,5.1 \mathrm{~Hz}, 1 \mathrm{H}), 2.53(\mathrm{br}, 1 \mathrm{H}), 2.34(\mathrm{br}, 1 \mathrm{H}), 1.65(\mathrm{~m}, 1 \mathrm{H}), 1.55(\mathrm{~m}, 1 \mathrm{H}), 1.36(\mathrm{~s}, 3 \mathrm{H}), 1.20(\mathrm{~s}, 3 \mathrm{H}), 1.06(\mathrm{t}, J$ $=7.4 \mathrm{~Hz}, 3 \mathrm{H}) ;{ }^{13} \mathrm{C} \mathrm{NMR}\left(\mathrm{CDCl}_{3}, 125 \mathrm{MHz}\right) \delta 139.1,128.4,127.4,127.2,84.5,82.6,81.9,80.5,68.4,65.6$, 23.1, 19.0, 12.5, 10.9; IR (KBr) 3416, 3342, $1060 \mathrm{~cm}^{-1} ;[\alpha]^{23}{ }_{\mathrm{D}}+28.9\left(c 1.09, \mathrm{CHCl}_{3}\right) ; \mathrm{HRMS}\left(\mathrm{CI} / \mathrm{NH}_{3}\right) \mathrm{m} / z$ 
calcd for $\mathrm{C}_{16} \mathrm{H}_{25} \mathrm{O}_{4}(\mathrm{M}+\mathrm{H})^{+}$281.1753, found 281.1747. Anal. Calcd for $\mathrm{C}_{16} \mathrm{H}_{24} \mathrm{O}_{4}: \mathrm{C}, 68.54 ; \mathrm{H}$, 8.63. Found: C, 68.78; H, 8.66.<smiles>CC[C@H]1O[C@](C)(CO)[C@@H](O)[C@@]1(O)Br</smiles>

40

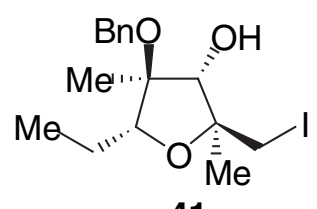

41

(2R,3R,4R,5R)-4-Benzyloxy-2,4-dimethyl-5-ethyl-3-hydroxy-2-iodomethyltetrahydrofuran (41). To a solution of 40 ( $4.35 \mathrm{~g}, 15.5 \mathrm{mmol})$ in $100 \mathrm{~mL}$ of benzene was added $\mathrm{Ph}_{3} \mathrm{P}(4.88 \mathrm{~g}, 18.6 \mathrm{mmol})$, imidazole (1.58 $\mathrm{g}, 23.2 \mathrm{mmol})$, and $\mathrm{I}_{2}(4.72 \mathrm{~g}, 18.6 \mathrm{mmol})$. The mixture was heated at reflux for $1 \mathrm{~h}$. After being cooled to 23 ${ }^{\circ} \mathrm{C}$, the mixture was filtered through Celite, and the cake was washed with $100 \mathrm{~mL}$ of ether. The filtrate was concentrated in vacuo. Purification by flash chromatography (5:95 to 10:90 EtOAc/hexanes) afforded $\mathbf{4 1}$ as a white solid (4.70 g, 78\%): $\mathrm{mp} 93-94{ }^{\circ} \mathrm{C} ;{ }^{1} \mathrm{H}$ NMR $\left(\mathrm{CDCl}_{3}, 500 \mathrm{MHz}\right) \delta 7.35(\mathrm{~m}, 5 \mathrm{H}), 4.74(\mathrm{~d}, J=11.7 \mathrm{~Hz}, 1 \mathrm{H})$, $4.71(\mathrm{~d}, J=11.7 \mathrm{~Hz}, 1 \mathrm{H}), 4.37(\mathrm{~d}, J=5.4 \mathrm{~Hz}, 1 \mathrm{H}), 3.74(\mathrm{dd}, J=8.9,4.9 \mathrm{~Hz}, 1 \mathrm{H}), 3.43(\mathrm{~d}, J=9.6 \mathrm{~Hz}, 1 \mathrm{H}), 3.33$ $(\mathrm{d}, J=9.6 \mathrm{~Hz}, 1 \mathrm{H}), 1.80(\mathrm{~d}, J=5.4 \mathrm{~Hz}, 1 \mathrm{H}), 1.65(\mathrm{~m}, 1 \mathrm{H}), 1.57(\mathrm{~m}, 1 \mathrm{H}), 1.41(\mathrm{~s}, 3 \mathrm{H}), 1.35(\mathrm{~s}, 3 \mathrm{H}), 1.06(\mathrm{t}, J=$ $7.4 \mathrm{~Hz}, 3 \mathrm{H}) ;{ }^{13} \mathrm{C}$ NMR $\left(\mathrm{CDCl}_{3}, 125 \mathrm{MHz}\right) \delta 139.2,128.4,127.4,127.2,84.7,83.6,82.2,80.4,65.8,22.5,21.5$, 17.3, 12.0, 10.9; IR (KBr) 3437, $1092 \mathrm{~cm}^{-1} ;[\alpha]^{23}{ }_{\mathrm{D}}-42.9\left(c\right.$ 1.06, $\left.\mathrm{CHCl}_{3}\right)$; HRMS $\left(\mathrm{CI}^{2} \mathrm{NH}_{3}\right) \mathrm{m} / z$ calcd for $\mathrm{C}_{16} \mathrm{H}_{27} \mathrm{NIO}_{3}\left(\mathrm{M}+\mathrm{NH}_{4}\right)^{+}$408.1036, found 408.1037. Anal. Calcd for $\mathrm{C}_{16} \mathrm{H}_{23} \mathrm{IO}_{3}: \mathrm{C}, 49.24 ; \mathrm{H}, 5.94$. Found: $\mathrm{C}$, $49.41 ; \mathrm{H}, 5.82$.<smiles>CC[C@H]1O[C@](C)(CI)[C@@H](O)[C@]1(C)OBr</smiles>

41

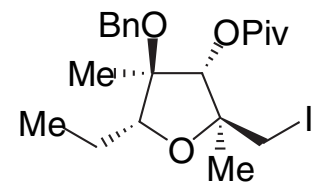

14

2,2-Dimethyl propionic acid $(2 R, 3 R, 4 R, 5 R)$-4-Benzyloxy-2,4-dimethyl-5-ethyl-2-iodomethyltetrahydrofuran-3-yl ester (14). To a mixture of alcohol $41(4.70 \mathrm{~g}, 12.1 \mathrm{mmol})$ and trimethylacetic anhydride $(12.2 \mathrm{~mL}$, $60.3 \mathrm{mmol})$ in acetonitrile $(60 \mathrm{~mL})$ was added dropwise a solution of $\mathrm{Sc}(\mathrm{OTf})_{3}(0.296 \mathrm{~g}, 0.600 \mathrm{mmol})$ in $10 \mathrm{~mL}$ of acetonitrile at $23{ }^{\circ} \mathrm{C}$. After $1 \mathrm{~h}, 50 \mathrm{~mL}$ of saturated aqueous $\mathrm{NaHCO}_{3}$ solution was added. The mixture was extracted with $3 \times 100 \mathrm{~mL}$ of ether. The combined organic layers were washed with brine $(50 \mathrm{~mL})$, dried $\left(\mathrm{MgSO}_{4}\right)$, and concentrated in vacuo. Purification by flash chromatography $\left(1: 99 \mathrm{Et}_{2} \mathrm{O} /\right.$ hexanes $)$ afforded $\mathbf{1 4}$ as a colorless oil $(5.72 \mathrm{~g}, 100 \%):{ }^{1} \mathrm{H} \mathrm{NMR}\left(\mathrm{CDCl}_{3}, 500 \mathrm{MHz}\right) \delta 7.37(\mathrm{~m}, 5 \mathrm{H}), 5.45(\mathrm{~s}, 1 \mathrm{H}), 4.60(\mathrm{~d}, J=11.0 \mathrm{~Hz}$, $1 \mathrm{H}), 4.46(\mathrm{~d}, J=11.0 \mathrm{~Hz}, 1 \mathrm{H}), 3.89(\mathrm{dd}, J=9.1,4.0 \mathrm{~Hz}, 1 \mathrm{H}), 3.66(\mathrm{~d}, J=10.5 \mathrm{~Hz}, 1 \mathrm{H}), 3.63(\mathrm{~d}, J=10.5 \mathrm{~Hz}$, $1 \mathrm{H}), 1.60(\mathrm{~m}, 2 \mathrm{H}), 1.37(\mathrm{~s}, 3 \mathrm{H}), 1.34(\mathrm{~s}, 9 \mathrm{H}), 1.30(\mathrm{~s}, 3 \mathrm{H}), 1.10(\mathrm{t}, J=7.4 \mathrm{~Hz}, 3 \mathrm{H}) ;{ }^{13} \mathrm{C} \mathrm{NMR}\left(\mathrm{CDCl}_{3}, 125\right.$ MHz) $\delta$ 177.1, 138.3, 128.4, 127.5, 127.4, 85.8, 83.7, 81.3, 80.4, 65.9, 39.0, 27.1, 22.9, 22.7, 16.1, 13.6, 10.9; IR (thin film) 1732, $1148 \mathrm{~cm}^{-1} ;[\alpha]^{23}{ }_{D}-2.0\left(c 0.99, \mathrm{CHCl}_{3}\right) ; \mathrm{HRMS}\left(\mathrm{CI} / \mathrm{NH}_{3}\right) \mathrm{m} / z$ calcd for $\mathrm{C}_{21} \mathrm{H}_{32} \mathrm{IO}_{4}(\mathrm{M}+\mathrm{H})^{+}$ 475.1345, found 475.1341. Anal. Calcd for $\mathrm{C}_{21} \mathrm{H}_{31} \mathrm{IO}_{4}$ : C, 53.17; H, 6.59. Found: C, 53.61; H, 6.78. 


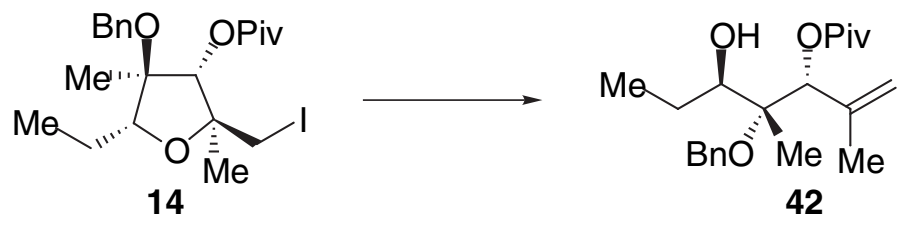

2,2-Dimethyl propionic acid $(R)$-[(1R,2R)-1-benzyloxy-1-methyl-2-hydroxybutyl)]-2-methylallyl ester (42). To a solution of the iodide $14(5.72 \mathrm{~g}, 12.0 \mathrm{mmol})$ in $30 \mathrm{~mL}$ of dry $\mathrm{Et}_{2} \mathrm{O}$ and $30 \mathrm{~mL}$ of dry $\mathrm{MeOH}$ was added zinc powder $(15.7 \mathrm{~g}, 24.0 \mathrm{mmol})$ followed by $\mathrm{AcOH}(2.23 \mathrm{~mL}, 36.0 \mathrm{mmol})$. The reaction mixture was stirred for $12 \mathrm{~h}$ at $23{ }^{\circ} \mathrm{C}$, at which point the reaction mixture was diluted with $400 \mathrm{~mL}$ of $\mathrm{Et}_{2} \mathrm{O}$ and filtered. The filtrate was washed with $50 \mathrm{~mL}$ of saturated aqueous $\mathrm{NaHCO}_{3}$ and brine $(50 \mathrm{~mL})$, dried $\left(\mathrm{MgSO}_{4}\right)$, and concentrated in vасио. Purification by flash chromatography (5:95 to 10:90 EtOAc/hexanes) afforded 42 as a white solid (3.45 g, 83\%): $\mathrm{mp} 54-55^{\circ} \mathrm{C} ;{ }^{1} \mathrm{H} \mathrm{NMR}\left(\mathrm{CDCl}_{3}, 500 \mathrm{MHz}\right) \delta 7.35(\mathrm{~m}, 5 \mathrm{H}), 5.51(\mathrm{~s}, 1 \mathrm{H}), 5.11(\mathrm{t}, J=1.5 \mathrm{~Hz}, 1 \mathrm{H}), 5.09$ $(\mathrm{s}, 1 \mathrm{H}), 4.63(\mathrm{~d}, J=11.2 \mathrm{~Hz}, 1 \mathrm{H}), 4.60(\mathrm{~d}, J=11.2 \mathrm{~Hz}, 1 \mathrm{H}), 3.68(\mathrm{ddd}, J=10.4,4.5,1.7 \mathrm{~Hz}, 1 \mathrm{H}), 2.57(\mathrm{dd}, J=$ 4.5, $1.2 \mathrm{~Hz}, 1 \mathrm{H}), 1.94(\mathrm{~s}, 3 \mathrm{H}), 1.74(\mathrm{~m}, 1 \mathrm{H}), 1.48(\mathrm{~m}, 1 \mathrm{H}), 1.35(\mathrm{~s}, 3 \mathrm{H}), 1.32(\mathrm{~s}, 9 \mathrm{H}), 1.09(\mathrm{t}, J=7.4 \mathrm{~Hz}, 3 \mathrm{H})$; ${ }^{13} \mathrm{C} \mathrm{NMR}\left(\mathrm{CDCl}_{3}, 125 \mathrm{MHz}\right) \delta 177.6,141.9,138.8,128.3,127.3,127.0,115.4,80.6,79.4,75.1,64.8,39.0$, 27.1, 23.7, 20.6, 16.5, 11.4; IR (thin film) 3520, 1732, $1150 \mathrm{~cm}^{-1} ;[\alpha]_{\mathrm{D}}^{23}+15.3\left(c 1.15, \mathrm{CHCl}_{3}\right) ; \mathrm{HRMS}$ $\left(\mathrm{CI} / \mathrm{NH}_{3}\right) \mathrm{m} / \mathrm{z}$ calcd for $\mathrm{C}_{21} \mathrm{H}_{32} \mathrm{O}_{4}(\mathrm{M}+\mathrm{H})^{+} 349.2379$, found 349.2383. Anal. Calcd for $\mathrm{C}_{21} \mathrm{H}_{32} \mathrm{O}_{4}: \mathrm{C}, 72.38 ; \mathrm{H}$, 9.26. Found: C, 72.66; H, 9.43.

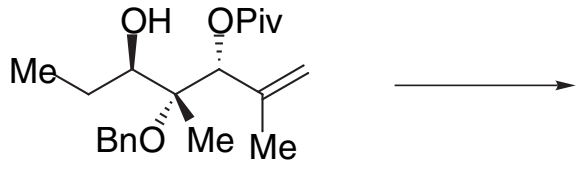

42

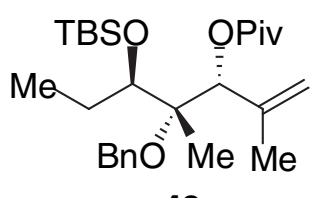

43

2,2-Dimethyl propionic acid $(R)$-[(1R,2R)-benzyloxy-1-methyl-tert-butyldimethylsiloxy-butyl)-2methylallyl ester (43). To a solution of alcohol $42(3.45 \mathrm{~g}, 10.0 \mathrm{mmol})$ was added 2,6-lutidine $(2.31 \mathrm{~mL}, 20.0$ mmol) and TBSOTf $(4.59 \mathrm{~mL}, 20.0 \mathrm{mmol})$ at $0{ }^{\circ} \mathrm{C}$. The reaction mixture was allowed to stir for $3 \mathrm{~h}$ at $23{ }^{\circ} \mathrm{C}$. The reaction mixture was diluted with $300 \mathrm{~mL}$ of $\mathrm{Et}_{2} \mathrm{O}$ and washed with saturated aqueous $\mathrm{NaHCO}_{3}(30 \mathrm{~mL})$ and brine $(30 \mathrm{~mL})$, dried $\left(\mathrm{MgSO}_{4}\right)$, and concentrated in vacuo. Purification by flash chromatography (2:98 $\mathrm{Et}_{2} \mathrm{O} /$ hexanes) afforded 43 as a colorless oil $(4.42 \mathrm{~g}, 97 \%):{ }^{1} \mathrm{H} \mathrm{NMR}\left(\mathrm{CDCl}_{3}, 500 \mathrm{MHz}\right) \delta 7.38(\mathrm{~m}, 4 \mathrm{H}), 7.31$ $(\mathrm{m}, 1 \mathrm{H}), 5.32(\mathrm{~s}, 1 \mathrm{H}), 5.11(\mathrm{~s}, 1 \mathrm{H}), 5.10(\mathrm{t}, J=1.5 \mathrm{~Hz}, 1 \mathrm{H}), 4.89(\mathrm{~d}, J=11.9 \mathrm{~Hz}, 1 \mathrm{H}), 4.71(\mathrm{~d}, J=11.9 \mathrm{~Hz}, 1 \mathrm{H})$, $3.85(\mathrm{dd}, J=7.4,2.7 \mathrm{~Hz}, 1 \mathrm{H}), 1.92(\mathrm{~m}, 1 \mathrm{H}$, and s, 3H), $1.60(\mathrm{~m}, 1 \mathrm{H}), 1.39(\mathrm{~s}, 3 \mathrm{H}), 1.30(\mathrm{~s}, 9 \mathrm{H}), 1.07(\mathrm{t}, J=7.5$ $\mathrm{Hz}, 3 \mathrm{H}), 1.01(\mathrm{~s}, 9 \mathrm{H}), 0.16(\mathrm{~s}, 3 \mathrm{H}), 0.13(\mathrm{~s}, 3 \mathrm{H}) ;{ }^{13} \mathrm{C}$ NMR $\left(\mathrm{CDCl}_{3}, 125 \mathrm{MHz}\right) \delta 177.0,142.1,139.8,128.1$, $126.8,126.6,116.1,81.9,80.0,76.9,65.9,38.9,27.2,26.1,25.4,21.0,18.3,16.1,12.0,-3.6,-4.2$; IR (thin film) 1733, $1149 \mathrm{~cm}^{-1} ;[\alpha]^{23}{ }_{\mathrm{D}}+6.1\left(c\right.$ 1.01, $\left.\mathrm{CHCl}_{3}\right) ; \mathrm{HRMS}\left(\mathrm{CI} / \mathrm{NH}_{3}\right) \mathrm{m} / z$ calcd for $\mathrm{C}_{27} \mathrm{H}_{47} \mathrm{O}_{4} \mathrm{Si}(\mathrm{M}+\mathrm{H})^{+}$ 463.3243, found 463.3244. Anal. Calcd for $\mathrm{C}_{27} \mathrm{H}_{46} \mathrm{O}_{4} \mathrm{Si}: \mathrm{C}, 70.08 ; \mathrm{H}, 10.02$. Found: C, 70.32; H, 10.13. 
<smiles>C=C(C)C(OC#N)C(C)(O)C(CC)OC(C)(C)C</smiles>

43

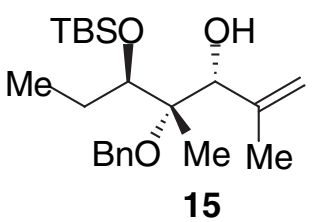

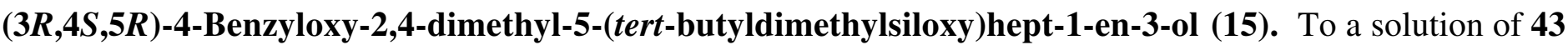
(4.47 g, $9.68 \mathrm{mmol})$ in $60 \mathrm{~mL}$ of $\mathrm{CH}_{2} \mathrm{Cl}_{2}$ was added $i-\mathrm{Bu}_{2} \mathrm{AlH}(25.8 \mathrm{~mL}, 1.5 \mathrm{M}$ solution in toluene, $38.7 \mathrm{mmol})$ dropwise at $-78{ }^{\circ} \mathrm{C}$. The reaction mixture was allowed to warm to $0{ }^{\circ} \mathrm{C}$. After being stirred at $0{ }^{\circ} \mathrm{C}$ for $3 \mathrm{~h}$, the mixture was cooled to $-78{ }^{\circ} \mathrm{C}$, followed by the addition of $5 \mathrm{~mL}$ of $\mathrm{MeOH}$ and $50 \mathrm{~mL}$ of a saturated aqueous solution of potassium sodium tartrate. The mixture was then stirred at $23{ }^{\circ} \mathrm{C}$ for $1 \mathrm{~h}$. The layers were separated, and the aqueous layer was extracted with $3 \times 50 \mathrm{~mL}$ of $\mathrm{CH}_{2} \mathrm{Cl}_{2}$. The combined organic layers were washed with brine $(30 \mathrm{~mL})$, dried $\left(\mathrm{MgSO}_{4}\right)$, and concentrated in vacuo. Purification by flash chromatography (5:95 $\mathrm{Et}_{2} \mathrm{O} /$ hexanes) afforded 15 as a colorless oil $(3.45 \mathrm{~g}, 94 \%):{ }^{1} \mathrm{H} \mathrm{NMR}\left(\mathrm{CDCl}_{3}, 500 \mathrm{MHz}\right) \delta 7.40(\mathrm{~m}, 4 \mathrm{H}), 7.35$ $(\mathrm{m}, 1 \mathrm{H}), 5.08(\mathrm{t}, J=1.6 \mathrm{~Hz}, 1 \mathrm{H}), 5.03(\mathrm{~s}, 1 \mathrm{H}), 4.78(\mathrm{~d}, J=11.2 \mathrm{~Hz}, 1 \mathrm{H}), 4.67(\mathrm{~d}, J=11.2 \mathrm{~Hz}, 1 \mathrm{H}), 4.27(\mathrm{~d}, J=$ $5.4 \mathrm{~Hz}, 1 \mathrm{H}), 3.93(\mathrm{dd}, J=7.2,3.3 \mathrm{~Hz}, 1 \mathrm{H}), 3.42(\mathrm{~d}, J=5.4 \mathrm{~Hz}, 1 \mathrm{H}), 1.92(\mathrm{~s}, 3 \mathrm{H}), 1.91(\mathrm{~m}, 1 \mathrm{H}), 1.68(\mathrm{~m}, 1 \mathrm{H})$, $1.32(\mathrm{~s}, 3 \mathrm{H}), 1.12(\mathrm{t}, J=7.5 \mathrm{~Hz}, 3 \mathrm{H}), 1.01(\mathrm{~s}, 9 \mathrm{H}), 0.20(\mathrm{~s}, 3 \mathrm{H}), 0.18(\mathrm{~s}, 3 \mathrm{H}) ;{ }^{13} \mathrm{C} \mathrm{NMR}\left(\mathrm{CDCl}_{3}, 125 \mathrm{MHz}\right) \delta$ 145.0, 139.0, 128.3, 127.2, 114.8, 81.6, 77.9, 77.6, 65.7, 26.1, 25.6, 19.6, 18.3, 15.7, 11.8, -3.8, -4.4; IR (thin film) $3555,1108 \mathrm{~cm}^{-1} ;[\alpha]^{23}{ }_{\mathrm{D}}-3.4\left(c\right.$ 1.02, $\left.\mathrm{CHCl}_{3}\right)$; HRMS $\left(\mathrm{CI} / \mathrm{NH}_{3}\right) \mathrm{m} / \mathrm{z}$ calcd for $\mathrm{C}_{22} \mathrm{H}_{39} \mathrm{O}_{3} \mathrm{Si}(\mathrm{M}+\mathrm{H})^{+}$ 379.2668, found 379.2671. Anal. Calcd for $\mathrm{C}_{22} \mathrm{H}_{38} \mathrm{O}_{3} \mathrm{Si}$ : C, 69.79; H, 10.12. Found: C, 69.95; H, 10.22.

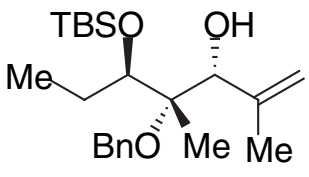

15

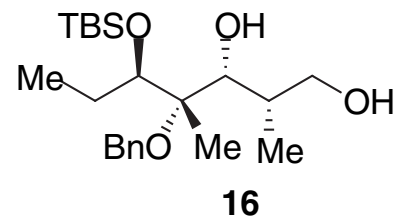

16

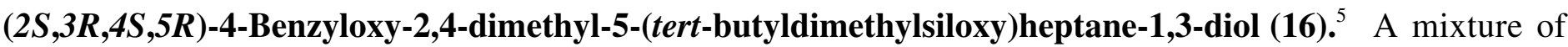
alcohol $15(3.40 \mathrm{~g}, 9.00 \mathrm{mmol})$ and $\mathrm{HN}\left(\mathrm{SiHMe}_{2}\right)(16.0 \mathrm{~mL}, 90.0 \mathrm{mmol})$ was placed in a $90{ }^{\circ} \mathrm{C}$ bath and allowed to stir for $24 \mathrm{~h}$. The reaction mixture was then cooled to $23{ }^{\circ} \mathrm{C}$ and was placed under high vacuum $(0.1 \mathrm{mmHg})$ for $4 \mathrm{~h}$ to remove the volatile materials. The residue was dissolved in $30 \mathrm{~mL}$ of toluene and platinum(0)-1,3divinyl-1,1,3,3-tetramethyldisiloxane complex $(0.5 \mathrm{~mL}$, in xylenes $)$ was added. The reaction mixture was stirred at $70{ }^{\circ} \mathrm{C}$ for $12 \mathrm{~h}$. After being cooled to $23{ }^{\circ} \mathrm{C}$, the mixture was diluted with $80 \mathrm{~mL}$ of hexanes and $10 \mathrm{~g}$ of EDTA disodium salt was added. The mixture was stirred for $12 \mathrm{~h}$, filtered, and concentrated in vacuo. The residue was dissolved in $40 \mathrm{~mL}$ of $\mathrm{MeOH}$ and $40 \mathrm{~mL}$ of THF. To this solution was added $\mathrm{KHCO}_{3}(1.80 \mathrm{~g}, 18.0$ mmol), $\mathrm{KF}(1.04 \mathrm{~g}, 18.0 \mathrm{mmol})$ and $\mathrm{H}_{2} \mathrm{O}_{2}(20.4 \mathrm{~mL}, 30 \%$ solution in water, $180 \mathrm{mmol})$. The resulting mixture was stirred at $23{ }^{\circ} \mathrm{C}$ for $12 \mathrm{~h}$ and was quenched by the addition of $30 \mathrm{~mL}$ of saturated aqueous $\mathrm{Na}_{2} \mathrm{~S}_{2} \mathrm{O}_{3}$ solution. The mixture was extracted with $4 \times 50 \mathrm{~mL}$ of EtOAc. The combined organic layers were washed with brine (30 $\mathrm{mL}$ ), dried $\left(\mathrm{MgSO}_{4}\right)$, and concentrated in vacuo. Purification by flash chromatography (5:95 to 20:80 EtOAc/hexanes) afforded a white solid $16(3.10 \mathrm{~g}, 87 \%)$ as a single diastereomer as determined by ${ }^{1} \mathrm{H}$ NMR spectroscopy analysis of the crude mixture: $\mathrm{mp} 84-85{ }^{\circ} \mathrm{C} ;{ }^{1} \mathrm{H}$ NMR $\left(\mathrm{CDCl}_{3}, 500 \mathrm{MHz}\right) \delta 7.39(\mathrm{~m}, 4 \mathrm{H}), 7.32(\mathrm{~m}$, $1 \mathrm{H}), 4.74(\mathrm{~d}, J=11.2 \mathrm{~Hz}, 1 \mathrm{H}), 4.64(\mathrm{~d}, J=11.2 \mathrm{~Hz}, 1 \mathrm{H}), 3.97$ (dd, $J=5.8,2.4 \mathrm{~Hz}, 1 \mathrm{H}), 3.93(\mathrm{dd}, J=6.7,3.8$ $\mathrm{Hz}, 1 \mathrm{H}), 3.73(\mathrm{dd}, J=10.4,3.8 \mathrm{~Hz}, 1 \mathrm{H}), 3.68(\mathrm{~m}, 1 \mathrm{H}), 3.40(\mathrm{~d}, J=5.7 \mathrm{~Hz}, 1 \mathrm{H}), 2.83(\mathrm{br}, 1 \mathrm{H}), 1.96(\mathrm{~m}, 1 \mathrm{H})$, $1.84(\mathrm{~m}, 1 \mathrm{H}), 1.64(\mathrm{~m}, 1 \mathrm{H}), 1.36(\mathrm{~s}, 3 \mathrm{H}), 1.12(\mathrm{~d}, J=7.0 \mathrm{~Hz}, 3 \mathrm{H}), 1.09$ (t, $J=7.5 \mathrm{~Hz}, 3 \mathrm{H}), 0.98(\mathrm{~s}, 9 \mathrm{H}), 0.19$ (s,

\footnotetext{
${ }^{5}$ Tamao, K. Nakajima, T.; Sumiya, R.; Arai, H.; Higuchi, N.; Ito, Y. J. Am. Chem. Soc. 1986, 108, 6090-6093.
} 
$3 \mathrm{H}), 0.16(\mathrm{~s}, 3 \mathrm{H}) ;{ }^{13} \mathrm{C} \mathrm{NMR}\left(\mathrm{CDCl}_{3}, 125 \mathrm{MHz}\right) \delta 138.8,128.3,127.3,81.5,78.2,75.6,68.4,65.1,36.1,26.0$, 25.7, 18.3, 16.1, 11.8, 11.1, -3.8, -4.4; IR (KBr) 3503, $1106 \mathrm{~cm}^{-1}$; $[\alpha]^{23}{ }_{\mathrm{D}}^{2}+15.9\left(c\right.$ 1.16, $\left.\mathrm{CHCl}_{3}\right)$; HRMS $\left(\mathrm{CI} / \mathrm{NH}_{3}\right) \mathrm{m} / z$ calcd for $\mathrm{C}_{22} \mathrm{H}_{41} \mathrm{O}{ }_{4} \mathrm{Si}(\mathrm{M}+\mathrm{H})^{+}$397.2774, found 397.2779. Anal. Calcd for $\mathrm{C}_{22} \mathrm{H}_{40} \mathrm{O} \mathrm{O}_{4} \mathrm{Si}$ : C, 66.62; H, 10.16. Found: C, 66.36; H, 10.31 .<smiles>CCOC(CC)C(O)C(C)(O)[C@H](O)[C@H](C)CO</smiles>

16

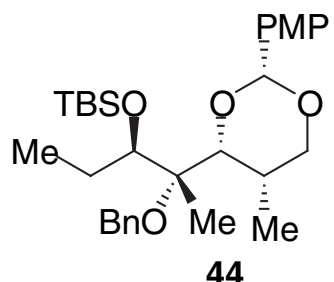

44

$(2 R, 4 R, 5 S)-4-[(1 S, 2 R)$-1-Benzyloxy-2-tert-butyldimethylsiloxy-1-methylbutyl)]-2-(p-methoxyphenyl)-5methyl-1,3-dioxane (44). To a solution of $16(3.06 \mathrm{~g}, 7.73 \mathrm{mmol})$ in $50 \mathrm{~mL}$ of $\mathrm{CH}_{2} \mathrm{Cl}_{2}$ was added $p$ anisaldehyde dimethylacetal $(1.84 \mathrm{~mL}, 10.8 \mathrm{mmol})$ and PPTS $(0.388 \mathrm{~g}, 1.55 \mathrm{mmol})$. After $5 \mathrm{~h}$ at $23{ }^{\circ} \mathrm{C}, 30 \mathrm{~mL}$ of saturated aqueous $\mathrm{NaHCO}_{3}$ was added to the mixture. The layers were separated and the organic layer was extracted with $3 \times 50 \mathrm{~mL}$ of $\mathrm{CH}_{2} \mathrm{Cl}_{2}$. The combined organic layers were washed with brine $(30 \mathrm{~mL})$, dried $\left(\mathrm{MgSO}_{4}\right)$, and concentrated in vacuo. Purification by flash chromatography (2:98 EtOAc/hexanes) afforded 44 as needles $(3.80 \mathrm{~g}, 96 \%)$ : $\mathrm{mp} 100-101{ }^{\circ} \mathrm{C} ;{ }^{1} \mathrm{H} \mathrm{NMR}\left(\mathrm{CDCl}_{3}, 500 \mathrm{MHz}\right) \delta 7.56(\mathrm{~m}, 2 \mathrm{H}), 7.41(\mathrm{~m}, 4 \mathrm{H}), 7.34(\mathrm{~m}$, $1 \mathrm{H}), 7.02(\mathrm{~m}, 2 \mathrm{H}), 5.60(\mathrm{~s}, 1 \mathrm{H}), 5.15(\mathrm{~d}, J=11.8 \mathrm{~Hz}, 1 \mathrm{H}), 4.57(\mathrm{~d}, J=11.8 \mathrm{~Hz}, 1 \mathrm{H}), 4.37(\mathrm{~d}, J=1.2 \mathrm{~Hz}, 1 \mathrm{H})$, $4.34(\mathrm{dd}, J=6.9,2.3 \mathrm{~Hz}, 1 \mathrm{H}), 4.15(\mathrm{dd}, J=11.2,2.2 \mathrm{~Hz}, 1 \mathrm{H}), 4.08(\mathrm{dd}, J=11.3,1.3 \mathrm{~Hz}, 1 \mathrm{H}), 3.91(\mathrm{~s}, 3 \mathrm{H}), 2.00$ $(\mathrm{m}, 1 \mathrm{H}), 1.97(\mathrm{~m}, 1 \mathrm{H}), 1.57(\mathrm{~m}, 1 \mathrm{H}$ and d, $J=6.9 \mathrm{~Hz}, 3 \mathrm{H}), 1.26(\mathrm{~s}, 3 \mathrm{H}), 1.14(\mathrm{t}, J=7.5 \mathrm{~Hz}, 3 \mathrm{H}), 1.06(\mathrm{~s}, 9 \mathrm{H})$, 0.22 (s, 3H), $0.20(\mathrm{~s}, 3 \mathrm{H}) ;{ }^{13} \mathrm{C} \mathrm{NMR}\left(\mathrm{CDCl}_{3}, 125 \mathrm{MHz}\right) \delta 159.8,140.1,131.7,128.1,127.4,127.2,126.9,113.6$, $102.4,81.6,81.3,75.5,73.5,66.2,55.2,30.2,26.2,24.2,18.4,17.0,12.7,12.1,-3.2,-4.3$; IR (thin film) 2956, $1614,1518,1249,1112 \mathrm{~cm}^{-1} ;[\alpha]^{23}{ }_{\mathrm{D}}+8.0\left(c 1.09, \mathrm{CHCl}_{3}\right) ; \mathrm{HRMS}\left(\mathrm{CI} / \mathrm{NH}_{3}\right) \mathrm{m} / z$ calcd for $\mathrm{C}_{30} \mathrm{H}_{47} \mathrm{O}_{5} \mathrm{Si}(\mathrm{M}+\mathrm{H})^{+}$ 515.3193, found 515.3192. Anal. Calcd for $\mathrm{C}_{30} \mathrm{H}_{46} \mathrm{O}_{5} \mathrm{Si}$ : C, 70.00; H, 9.01. Found: C, 70.38; H, 9.02.

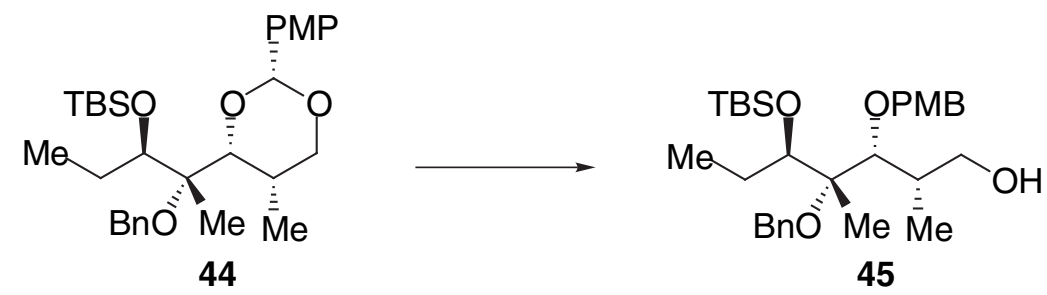

(2S,3R,4S,5R)-4-Benzyloxy-5-(tert-butyldimethylsiloxy)-2,4-dimethyl-3-(p-methoxybenzyloxy)-1-heptanol (45). To a solution of $44(3.90 \mathrm{~g}, 7.59 \mathrm{mmol})$ in $70 \mathrm{~mL}$ of $\mathrm{CH}_{2} \mathrm{Cl}_{2}$ was added $i$ - $\mathrm{Bu}_{2} \mathrm{AlH}(15.2 \mathrm{~mL}, 1.5 \mathrm{M}$ solution in toluene, $22.8 \mathrm{mmol}$ ) dropwise at $-78{ }^{\circ} \mathrm{C}$. The reaction mixture was allowed to warm to $0{ }^{\circ} \mathrm{C}$. After being stirred at $0{ }^{\circ} \mathrm{C}$ for $0.5 \mathrm{~h}$, the mixture was cooled to $-78{ }^{\circ} \mathrm{C}$, followed by the addition of $5 \mathrm{~mL}$ of MeOH followed by $50 \mathrm{~mL}$ of a saturated aqueous solution of potassium sodium tartrate. The mixture was then stirred at $23{ }^{\circ} \mathrm{C}$ for $1 \mathrm{~h}$. The layers were separated, the aqueous layer was extracted with $3 \times 50 \mathrm{~mL}$ of $\mathrm{CH}_{2} \mathrm{Cl}_{2}$. The combined organic layers were washed with brine $(30 \mathrm{~mL})$, dried $\left(\mathrm{MgSO}_{4}\right)$, and concentrated in vacuo. Purification by flash chromatography (10:90 to 15:85 EtOAc/hexanes) afforded $\mathbf{4 5}$ as a colorless oil (3.85 g, 98\%): ${ }^{1} \mathrm{H}$ NMR $\left(\mathrm{CDCl}_{3}, 500 \mathrm{MHz}\right) \delta 7.46-7.29(\mathrm{~m}, 7 \mathrm{H}), 6.94(\mathrm{~m}, 2 \mathrm{H}), 4.97(\mathrm{~d}, J=11.4 \mathrm{~Hz}, 1 \mathrm{H}), 4.90(\mathrm{~d}, J=$ $11.2 \mathrm{~Hz}, 1 \mathrm{H}), 4.75(\mathrm{~d}, J=11.4 \mathrm{~Hz}, 1 \mathrm{H}), 4.62(\mathrm{~d}, J=11.2 \mathrm{~Hz}, 1 \mathrm{H}), 3.87(\mathrm{~s}, 3 \mathrm{H}), 3.86(\mathrm{~d}, J=2.1 \mathrm{~Hz}, 1 \mathrm{H}), 3.77$ (dd, $J=6.6,3.8 \mathrm{~Hz}, 1 \mathrm{H}), 3.55(\mathrm{~m}, 2 \mathrm{H}), 1.91(\mathrm{~m}, 2 \mathrm{H}), 1.79(\mathrm{~m}, 2 \mathrm{H}), 1.53(\mathrm{~s}, 3 \mathrm{H}), 1.12(\mathrm{t}, J=7.5 \mathrm{~Hz}, 3 \mathrm{H}), 1.07$ 
$(\mathrm{d}, J=7.0 \mathrm{~Hz}, 3 \mathrm{H}), 1.06(\mathrm{~s}, 9 \mathrm{H}), 0.22(\mathrm{~s}, 3 \mathrm{H}), 0.20(\mathrm{~s}, 3 \mathrm{H}) ;{ }^{13} \mathrm{C} \mathrm{NMR}\left(\mathrm{CDCl}_{3}, 125 \mathrm{MHz}\right) \delta 158.9,140.2,131.4$, 129.3, 128.2, 127.6, 126.9, 113.6, 84.7, 83.1, 78.8, 74.5, 67.5, 65.8, 55.2, 36.8, 26.2, 25.5, 18.4, 16.8, 11.6, 11.4,

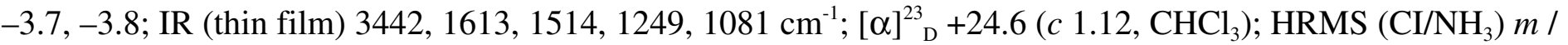
$z$ calcd for $\mathrm{C}_{30} \mathrm{H}_{49} \mathrm{O}_{5} \mathrm{Si}(\mathrm{M}+\mathrm{H})^{+}$517.3349, found 517.3349. Anal. Calcd for $\mathrm{C}_{30} \mathrm{H}_{48} \mathrm{O}_{5} \mathrm{Si}: \mathrm{C}, 69.72 ; \mathrm{H}, 9.36$. Found: C, 69.45; H, 9.59.

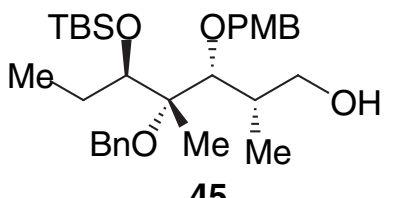

45

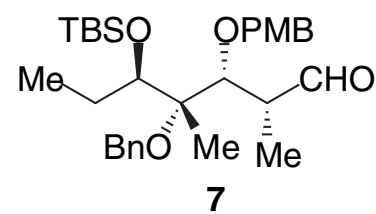

7

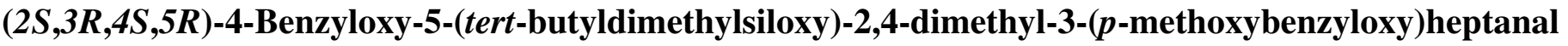

(7). To a solution of oxalyl chloride $\left(2.40 \mathrm{~mL}, 2.0 \mathrm{M}\right.$ solution in $\left.\mathrm{CH}_{2} \mathrm{Cl}_{2}, 4.80 \mathrm{mmol}\right)$ in $20 \mathrm{~mL}$ of $\mathrm{CH}_{2} \mathrm{Cl}_{2}$ at $-78{ }^{\circ} \mathrm{C}$ was added a solution of DMSO $(0.680 \mathrm{~mL}, 9.60 \mathrm{mmol})$ in $5 \mathrm{~mL}$ of $\mathrm{CH}_{2} \mathrm{Cl}_{2}$. The mixture was stirred at $-78{ }^{\circ} \mathrm{C}$ for $10 \mathrm{~min}$, and then a solution of alcohol $45(2.06 \mathrm{~g}, 4.00 \mathrm{mmol})$ in $15 \mathrm{~mL}$ of $\mathrm{CH}_{2} \mathrm{Cl}_{2}$ was added dropwise by syringe. After $30 \mathrm{~min}$ at $-78{ }^{\circ} \mathrm{C}, 1.67 \mathrm{~mL}(12.0 \mathrm{mmol})$ of $\mathrm{Et}_{3} \mathrm{~N}$ was added. The mixture was allowed to warm to $-10^{\circ} \mathrm{C}$ over $1 \mathrm{~h}$ and was then poured into $40 \mathrm{~mL}$ of water. The layers were separated, and the aqueous layer was extracted with $3 \times 40 \mathrm{~mL}$ of $\mathrm{CH}_{2} \mathrm{Cl}_{2}$. The combined organic layers were washed with brine $(30 \mathrm{~mL})$, dried $\left(\mathrm{MgSO}_{4}\right)$, and concentrated in vacuo. Purification by flash chromatography (5:95 EtOAc/hexanes) afforded 7 as a colorless oil $(2.04 \mathrm{~g}, 99 \%):{ }^{1} \mathrm{H} \mathrm{NMR}\left(\mathrm{CDCl}_{3}, 500 \mathrm{MHz}\right) \delta 9.68(\mathrm{~d}, J=1.0 \mathrm{~Hz}$, $1 \mathrm{H}), 7.38(\mathrm{~m}, 4 \mathrm{H}), 7.32(\mathrm{~m}, 1 \mathrm{H}), 7.25(\mathrm{~d}, J=8.7 \mathrm{~Hz}, 2 \mathrm{H}), 6.92(\mathrm{~m}, 2 \mathrm{H}), 4.84(\mathrm{~d}, J=11.4 \mathrm{~Hz}, 1 \mathrm{H}), 4.63(\mathrm{~d}, J=$ $11.4 \mathrm{~Hz}, 1 \mathrm{H}), 4.61(\mathrm{~d}, J=11.0 \mathrm{~Hz}, 1 \mathrm{H}), 4.49(\mathrm{~d}, J=11.0 \mathrm{~Hz}, 1 \mathrm{H}), 4.23(\mathrm{~d}, J=3.5 \mathrm{~Hz}, 1 \mathrm{H}), 3.87(\mathrm{~s}, 3 \mathrm{H}), 3.88$ $(\mathrm{dd}, J=7.7,2.7 \mathrm{~Hz}, 1 \mathrm{H}), 2.82(\mathrm{~m}, 1 \mathrm{H}), 1.87(\mathrm{~m}, 1 \mathrm{H}), 1.65(\mathrm{~m}, 1 \mathrm{H}), 1.41(\mathrm{~s}, 3 \mathrm{H}), 1.31(\mathrm{~d}, J=7.2 \mathrm{~Hz}, 3 \mathrm{H}), 1.08$ (t, $J=7.5 \mathrm{~Hz}, 3 \mathrm{H}), 0.99(\mathrm{~s}, 9 \mathrm{H}), 0.15(\mathrm{~s}, 3 \mathrm{H}), 0.12(\mathrm{~s}, 3 \mathrm{H}) ;{ }^{13} \mathrm{C} \mathrm{NMR}\left(\mathrm{CDCl}_{3}, 125 \mathrm{MHz}\right) \delta 203.9,159.0,139.6$, 130.6, 129.1, 128.2, 127.5, 127.1, 113.6, 83.3, 81.4, 78.2, 74.0, 65.7, 55.2, 47.7, 26.2, 25.9, 18.45, 18.41, 12.0,

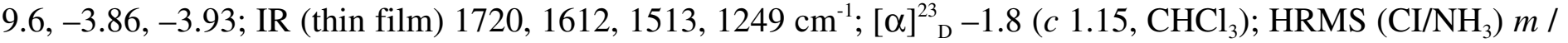
$z$ calcd for $\mathrm{C}_{30} \mathrm{H}_{45} \mathrm{O}_{5} \mathrm{Si}(\mathrm{M}-\mathrm{H})^{+}$513.3037, found 513.3036.
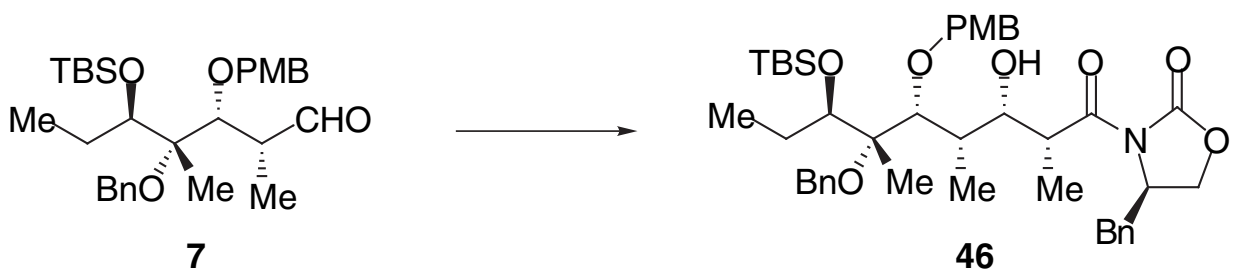

(4R)-4-Benzyl-3-[(2R,3S,4S,5R,6S,7R)-3-(6-benzyloxy-7-tert-butyldimethylsiloxy-3-hydroxy-5- $(p$ methoxyphenylmethoxy)-1-oxo-2,4,6-trimethylnonyl)]-2-oxazolidinone (46). ${ }^{3}$ To a solution $(R)$-4-benzyl-3propionyloxazolidin-2-one $(0.867 \mathrm{~g}, 3.72 \mathrm{mmol})$ in $20 \mathrm{~mL}$ of $\mathrm{CH}_{2} \mathrm{Cl}_{2}$ was added $\mathrm{TiCl}_{4}(0.428 \mathrm{~mL}, 3.91 \mathrm{mmol})$ at $0{ }^{\circ} \mathrm{C}$. After $5 \mathrm{~min}$, a solution of (-)-sparteine $(2.14 \mathrm{~mL}, 9.3 \mathrm{mmol})$ in $10 \mathrm{~mL}$ of $\mathrm{CH}_{2} \mathrm{Cl}_{2}$ was added slowly dropwise. After complete addition, the mixture was stirred for $20 \mathrm{~min}$ at $0{ }^{\circ} \mathrm{C}$. The mixture was cooled to -78 ${ }^{\circ} \mathrm{C}$, and aldehyde 7 (1.91 g, $\left.3.72 \mathrm{mmol}\right)$ in $10 \mathrm{~mL}$ of $\mathrm{CH}_{2} \mathrm{Cl}_{2}$ was added dropwsie. The mixture was stirred for 1 $\mathrm{h}$ at $-78{ }^{\circ} \mathrm{C}$, gradually warmed to $0{ }^{\circ} \mathrm{C}$, and stirred for $1 \mathrm{~h}$ at $0{ }^{\circ} \mathrm{C}$. To the mixture was added $40 \mathrm{~mL}$ of a halfsaturated aqueous solution of $\mathrm{NH}_{4} \mathrm{Cl}$. The layers were separated, and the aqueous layer was extracted with $3 \times$ $50 \mathrm{~mL}$ of $\mathrm{CH}_{2} \mathrm{Cl}_{2}$. The combined organic layers were washed with brine $(30 \mathrm{~mL})$, dried $\left(\mathrm{MgSO}_{4}\right)$, and concentrated in vacuo. Purification by flash chromatography (10:90 to 15:85 EtOAc/hexanes) afforded $\mathbf{4 6}$ as a 
foam (2.61 g, 94\%): ${ }^{1} \mathrm{H}$ NMR $\left(\mathrm{CDCl}_{3}, 500 \mathrm{MHz}\right) \delta 7.42-7.24(\mathrm{~m}, 12 \mathrm{H}), 6.88(\mathrm{~m}, 2 \mathrm{H}), 4.99(\mathrm{~d}, J=11.5 \mathrm{~Hz}$, $1 \mathrm{H}), 4.96(\mathrm{~d}, J=11.3 \mathrm{~Hz}, 1 \mathrm{H}), 4.70(\mathrm{~d}, J=11.5 \mathrm{~Hz}, 1 \mathrm{H}), 4.64(\mathrm{~m}, 1 \mathrm{H}), 4.58(\mathrm{~d}, J=11.3 \mathrm{~Hz}, 1 \mathrm{H}), 4.23(\mathrm{~d}, J=$ $5.3 \mathrm{~Hz}, 2 \mathrm{H}), 4.04(\mathrm{dt}, J=7.6,2.4 \mathrm{~Hz}, 1 \mathrm{H}), 3.86(\mathrm{~m}, 1 \mathrm{H}), 3.85(\mathrm{~s}, 3 \mathrm{H}), 3.82(\mathrm{~d}, J=2.2 \mathrm{~Hz}, 1 \mathrm{H}), 3.61(\mathrm{dd}, J=$ 7.4, $2.7 \mathrm{~Hz}, 1 \mathrm{H}), 3.29$ (dd, $J=13.4,3.2 \mathrm{~Hz}, 1 \mathrm{H}), 3.19$ (d, $J=1.8 \mathrm{~Hz}, 1 \mathrm{H}), 2.81(\mathrm{dd}, J=13.4,9.5 \mathrm{~Hz}, 1 \mathrm{H}), 1.70$ $(\mathrm{m}, 3 \mathrm{H}), 1.50(\mathrm{~s}, 3 \mathrm{H}), 1.36(\mathrm{~d}, J=6.9 \mathrm{~Hz}, 3 \mathrm{H}), 1.06(\mathrm{~d}, J=7.0 \mathrm{~Hz}, 3 \mathrm{H}), 1.05(\mathrm{t}, J=7.5 \mathrm{~Hz}, 3 \mathrm{H}), 1.02(\mathrm{~s}, 9 \mathrm{H})$, $0.19(\mathrm{~s}, 3 \mathrm{H}), 0.16(\mathrm{~s}, 3 \mathrm{H}) ;{ }^{13} \mathrm{C} \mathrm{NMR}\left(\mathrm{CDCl}_{3}, 125 \mathrm{MHz}\right) \delta 176.3,158.9,152.6,140.1,135.0,131.1,129.34$, $129.29,128.9,128.1,127.5,127.4,126.9,113.7,87.5,84.5,79.3,76.0,74.2,66.0,65.7,55.2,55.0,40.8,37.7$, 37.0, 26.2, 25.7, 18.4, 16.2, 14.0, 11.8, 8.7, -3.57, -3.62; IR (thin film) 3521, 1789, 1684, 1613, 1514, 1249, $1048 \mathrm{~cm}^{-1} ;[\alpha]^{23}{ }_{\mathrm{D}}-14.3\left(c\right.$ 1.40, $\mathrm{CHCl}_{3}$ ); HRMS (ESI) $m / z$ calcd for $\mathrm{C}_{43} \mathrm{H}_{61} \mathrm{NO}_{8} \mathrm{SiNa}(\mathrm{M}+\mathrm{Na})^{+} 770.4064$, found 770.4077. Anal. Calcd for $\mathrm{C}_{43} \mathrm{H}_{61} \mathrm{NO}_{8} \mathrm{Si}$ : C, 69.04; H, 8.22; N, 1.87. Found: C, 68.93; H, 8.23; N, 1.91.

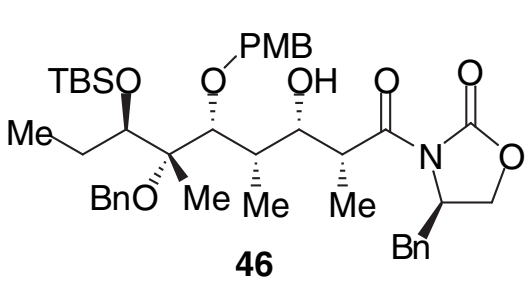

46

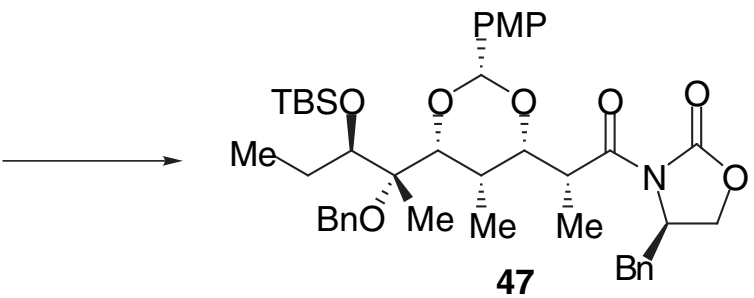

47

(4R)-4-Benzyl-3-[(2R)-2-[(2R,4S,5S,6R)-6-[(1S,2R)-1-benzyloxy-2-(tert-butyldimethylsiloxy)-1methylbutyl]-2-(p-methoxyphenyl)-5-methyl-1,3-dioxan-4-yl]propionyl]-2-oxazolidinone (47). To a mixture of $46(2.53 \mathrm{~g}, 3.39 \mathrm{mmol})$ and $4 \mathrm{~g}$ of activated $4 \AA$ molecular sieves powder in $20 \mathrm{~mL}$ of $\mathrm{CH}_{2} \mathrm{Cl}_{2} \mathrm{was}$ added a solution of DDQ $(0.920 \mathrm{~g}, 4.07 \mathrm{mmol})$ in $60 \mathrm{~mL}$ of $\mathrm{CH}_{2} \mathrm{Cl}_{2}$ at $0{ }^{\circ} \mathrm{C}$ by cannula. After $1 \mathrm{~h}$, the mixture was filtered through Celite, and the filtrate was washed with $40 \mathrm{~mL}$ of saturated aqueous $\mathrm{NaHCO}_{3}$ solution. The aqueous layer was back-extracted with $2 \times 30 \mathrm{~mL}$ of $\mathrm{CH}_{2} \mathrm{Cl}_{2}$. The combined organic layers were washed with brine $(30 \mathrm{~mL})$, dried $\left(\mathrm{MgSO}_{4}\right)$, and concentrated in vacuo. Purification by flash chromatography (10:90 to 15:85 EtOAc/hexanes) afforded 47 as a foam $(2.30 \mathrm{~g}, 91 \%):{ }^{1} \mathrm{H} \mathrm{NMR}\left(\mathrm{CDCl}_{3}, 500 \mathrm{MHz}\right) \delta 7.54(\mathrm{~d}, J=8.7 \mathrm{~Hz}$, $2 \mathrm{H}), 7.35(\mathrm{~m}, 10 \mathrm{H}), 7.00(\mathrm{~d}, J=8.7 \mathrm{~Hz}, 2 \mathrm{H}), 5.62(\mathrm{~s}, 1 \mathrm{H}), 5.08(\mathrm{~d}, J=11.7 \mathrm{~Hz}, 1 \mathrm{H}), 4.80(\mathrm{~m}, 1 \mathrm{H}), 4.53(\mathrm{~d}, J=$ $11.7 \mathrm{~Hz}, 1 \mathrm{H}), 4.38(\mathrm{dd}, J=6.5,2.1 \mathrm{~Hz}, 1 \mathrm{H}), 4.30-4.13(\mathrm{~m}, 5 \mathrm{H}), 3.90(\mathrm{~s}, 3 \mathrm{H}), 3.32(\mathrm{dd}, J=13.4,3.2 \mathrm{~Hz}, 1 \mathrm{H})$, $2.85(\mathrm{dd}, J=13.4,9.6 \mathrm{~Hz}, 1 \mathrm{H}), 2.08(\mathrm{~m}, 1 \mathrm{H}), 2.02(\mathrm{~m}, 1 \mathrm{H}), 1.54(\mathrm{~m}, 1 \mathrm{H}), 1.48(\mathrm{~d}, J=6.3 \mathrm{~Hz}, 3 \mathrm{H}), 1.30(\mathrm{~d}, J=$ $7.0 \mathrm{~Hz}, 3 \mathrm{H}), 1.22(\mathrm{~s}, 3 \mathrm{H}), 1.13(\mathrm{t}, J=7.5 \mathrm{~Hz}, 3 \mathrm{H}), 1.04(\mathrm{~s}, 9 \mathrm{H}), 0.20(\mathrm{~s}, 3 \mathrm{H}), 0.19(\mathrm{~s}, 3 \mathrm{H}) ;{ }^{13} \mathrm{C} \mathrm{NMR}\left(\mathrm{CDCl}_{3}\right.$, $125 \mathrm{MHz}) \delta 174.7,159.8,152.6,140.0,135.0,131.6,129.4,129.0,128.1,127.41,127.36,127.3,126.9,113.6$, 102.1, 83.0, 82.2, 81.4, 73.5, 66.2, 66.0, 55.3, 55.0, 39.7, 37.7, 30.9, 26.3, 24.3, 18.4, 17.3, 15.9, 12.2, 7.7, -3.3, -4.1; IR (thin film) 1782, 1695, 1615, 1518, 1248, $1051 \mathrm{~cm}^{-1} ;[\alpha]_{\mathrm{D}}^{23}-42.1\left(c 1.01, \mathrm{CHCl}_{3}\right) ; \mathrm{HRMS}(\mathrm{ESI}) \mathrm{m} / z$ calcd for $\mathrm{C}_{43} \mathrm{H}_{59} \mathrm{NO}_{8} \mathrm{SiNa}(\mathrm{M}+\mathrm{Na})^{+}$768.3907, found 768.3918. Anal. Calcd for $\mathrm{C}_{43} \mathrm{H}_{59} \mathrm{NO}_{8} \mathrm{Si}: \mathrm{C}, 69.23 ; \mathrm{H}$, 7.97; N, 1.88. Found: C, 69.40; H, 8.07; N, 1.85.
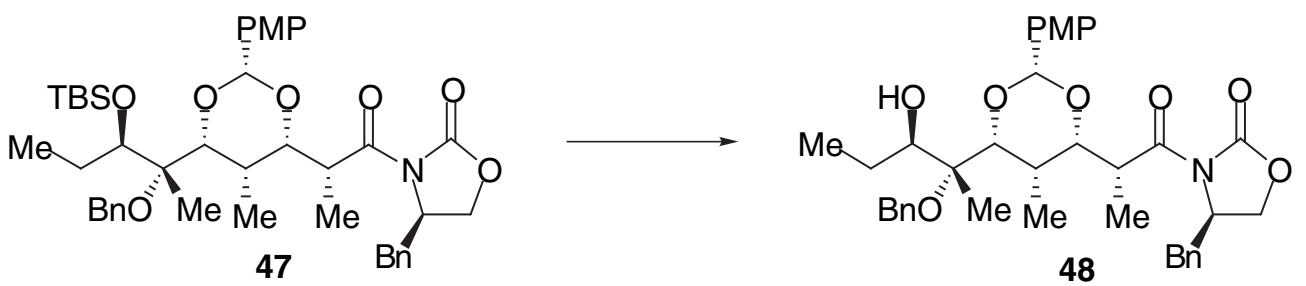

(4R)-4-Benzyl-3-[(2R)-2-[(2R,4S,5S,6R)-6-[(1S,2R)-1-benzyloxy-2-hydroxy-1-methylbutyl]-2-( $p$ methoxyphenyl)-5-methyl-1,3-dioxan-4-yl]propionyl]-2-oxazolidinone (48). To a solution of 47 (0.990 g, $1.33 \mathrm{mmol}$ ) in $10 \mathrm{~mL}$ of THF was added $3 \mathrm{~mL}$ of pyridine and $4 \mathrm{~mL}$ of HF.Py complex. After being stirred at 
$23{ }^{\circ} \mathrm{C}$ for 3 days, the reaction mixture was quenched by the slow addition of $40 \mathrm{~mL}$ of saturated aqueous $\mathrm{NaHCO}_{3}$ solution. The mixture was then extracted with $3 \times 100 \mathrm{~mL}$ of EtOAc. The combined organic layers were washed with brine $(30 \mathrm{~mL})$, dried $\left(\mathrm{MgSO}_{4}\right)$, and concentrated in vacuo. Purification by flash chromatography (20:80 to 30:70 EtOAc/hexanes) afforded 48 as a foam $(0.790 \mathrm{~g}, 94 \%):{ }^{1} \mathrm{H} \mathrm{NMR}\left(\mathrm{CDCl}_{3}, 500\right.$ MHz) $\delta 7.49(\mathrm{~d}, J=8.7 \mathrm{~Hz}, 2 \mathrm{H}), 7.37(\mathrm{~m}, 10 \mathrm{H}), 6.97(\mathrm{~d}, J=8.7 \mathrm{~Hz}, 2 \mathrm{H}), 5.61(\mathrm{~s}, 1 \mathrm{H}), 4.84(\mathrm{~d}, J=11.4 \mathrm{~Hz}$, $1 \mathrm{H}), 4.74(\mathrm{~m}, 1 \mathrm{H}), 4.62(\mathrm{~d}, J=11.4 \mathrm{~Hz}, 1 \mathrm{H}), 4.30-4.12(\mathrm{~m}, 5 \mathrm{H}), 3.87(\mathrm{~m}, 1 \mathrm{H}$ and s, 3H), $3.30(\mathrm{dd}, J=13.4,3.3$ $\mathrm{Hz}, 1 \mathrm{H}), 2.83(\mathrm{dd}, J=13.4,9.5 \mathrm{~Hz}, 1 \mathrm{H}), 2.70(\mathrm{br}, 1 \mathrm{H}), 2.01(\mathrm{~m}, 1 \mathrm{H}), 1.73(\mathrm{~m}, 1 \mathrm{H}), 1.53(\mathrm{~m}, 1 \mathrm{H}), 1.45(\mathrm{~d}, J=$ $6.3 \mathrm{~Hz}, 3 \mathrm{H}), 1.36(\mathrm{~s}, 3 \mathrm{H}), 1.22(\mathrm{~d}, J=7.0 \mathrm{~Hz}, 3 \mathrm{H}), 1.15(\mathrm{t}, J=7.3 \mathrm{~Hz}, 3 \mathrm{H}) ;{ }^{13} \mathrm{C} \mathrm{NMR}\left(\mathrm{CDCl}_{3}, 125 \mathrm{MHz}\right) \delta$ $174.7,160.0,152.5,139.6,135.0,130.9,129.4,129.0,128.1,127.4,127.33,127.26,127.0,113.6,102.2,84.7$, 83.2, 80.0, 75.9, 66.0, 64.9, 55.3, 55.0, 39.6, 37.7, 31.0, 25.3, 17.7, 15.8, 11.5, 8.5; IR (thin film) 3532, 1774, 1686, 1617, 1518, 1212, $1112 \mathrm{~cm}^{-1} ;[\alpha]^{23}-38.2$ ( $c$ 1.35, $\mathrm{CHCl}_{3}$ ); HRMS (ESI) $m / z$ calcd for $\mathrm{C}_{37} \mathrm{H}_{45} \mathrm{NO}_{8} \mathrm{SiNa}$ $(\mathrm{M}+\mathrm{Na})^{+}$654.3043, found 654.3037. Anal. Calcd for $\mathrm{C}_{37} \mathrm{H}_{45} \mathrm{NO}_{8}: \mathrm{C}, 70.34 ; \mathrm{H}, 7.18 ; \mathrm{N}, 2.22$. Found: C, $70.19 ; \mathrm{H}, 7.21 ; \mathrm{N}, 2.23$.
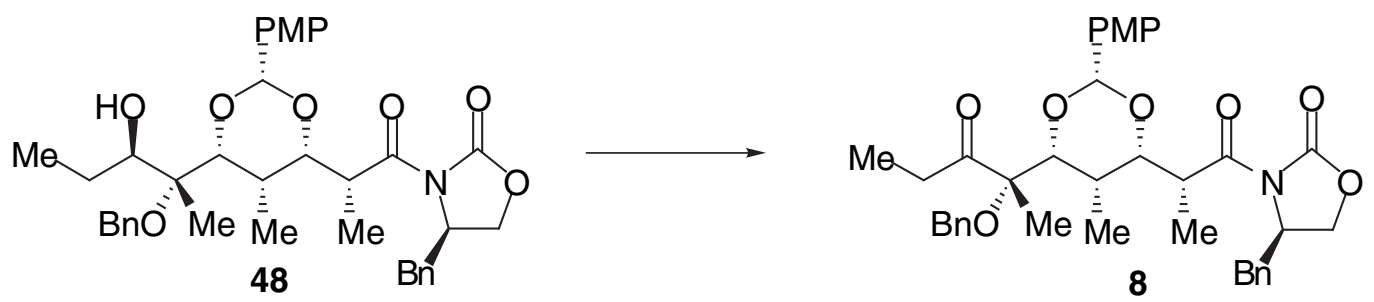

(4R)-4-Benzyl-3-[(2R)-2-[(2R,4S,5S,6R)-6-[(1S)-1-benzyloxy-1-methyl-2-oxobutyl]-2-(p-methoxyphenyl)-5methyl-1,3-dioxan-4-yl]propionyl]-2-oxazolidinone (8). To a solution of oxalyl chloride $(0.810 \mathrm{~mL}, 2.0 \mathrm{M}$ solution in $\left.\mathrm{CH}_{2} \mathrm{Cl}_{2}, 1.62 \mathrm{mmol}\right)$ in $12 \mathrm{~mL}$ of $\mathrm{CH}_{2} \mathrm{Cl}_{2}$ at $-78{ }^{\circ} \mathrm{C}$ was added a solution of DMSO $(0.230 \mathrm{~mL}, 3.24$ $\mathrm{mmol}$ ) in $2 \mathrm{~mL}$ of $\mathrm{CH}_{2} \mathrm{Cl}_{2}$. The mixture was stirred at $-78{ }^{\circ} \mathrm{C}$ for $10 \mathrm{~min}$, and then a solution of alcohol 48 $(0.850 \mathrm{~g}, 1.35 \mathrm{mmol})$ in $6 \mathrm{~mL}$ of $\mathrm{CH}_{2} \mathrm{Cl}_{2}$ was added dropwise by syringe. After $30 \mathrm{~min}$ at $-78{ }^{\circ} \mathrm{C}$, the mixture was allowed to warm to $-20{ }^{\circ} \mathrm{C}$ and stirred for $1 \mathrm{~h}$. The reaction mixture was then cooled to $-78{ }^{\circ} \mathrm{C}$, and 0.940 $\mathrm{mL}(6.75 \mathrm{mmol})$ of $\mathrm{Et}_{3} \mathrm{~N}$ was added. The mixture was allowed to warm to $-10{ }^{\circ} \mathrm{C}$ over $1 \mathrm{~h}$ and was then poured into $30 \mathrm{~mL}$ of water. The layers were separated, and the aqueous layer was extracted with $3 \times 40 \mathrm{~mL}$ of $\mathrm{CH}_{2} \mathrm{Cl}_{2}$. The combined organic layers were washed with brine $(30 \mathrm{~mL})$, dried $\left(\mathrm{MgSO}_{4}\right)$, and concentrated in vacuo. Purification by flash chromatography (15:85 to 20:80 EtOAc/hexanes) afforded 8 as a foam $(0.740 \mathrm{~g}$, 87\%): ${ }^{1} \mathrm{H} \mathrm{NMR}\left(\mathrm{CDCl}_{3}, 500 \mathrm{MHz}\right) \delta 7.47-7.27(\mathrm{~m}, 12 \mathrm{H}), 6.96(\mathrm{~m}, 2 \mathrm{H}), 5.58(\mathrm{~s}, 1 \mathrm{H}), 4.75(\mathrm{~m}, 1 \mathrm{H}), 4.64(\mathrm{~s}$, $2 \mathrm{H}), 4.30-4.11(\mathrm{~m}, 5 \mathrm{H}), 3.87(\mathrm{~s}, 3 \mathrm{H}), 3.29(\mathrm{dd}, J=13.4,3.2 \mathrm{~Hz}, 1 \mathrm{H}), 2.85-2.68(\mathrm{~m}, 3 \mathrm{H}), 1.99(\mathrm{~m}, 1 \mathrm{H}), 1.49(\mathrm{~s}$, $3 \mathrm{H}), 1.44(\mathrm{~d}, J=6.4 \mathrm{~Hz}, 3 \mathrm{H}), 1.15(\mathrm{~d}, J=7.0 \mathrm{~Hz}, 3 \mathrm{H}), 1.08(\mathrm{t}, J=7.2 \mathrm{~Hz}, 3 \mathrm{H}) ;{ }^{13} \mathrm{C} \mathrm{NMR}\left(\mathrm{CDCl}_{3}, 125 \mathrm{MHz}\right) \delta$ $215.1,174.5,159.8,152.5,138.8,135.0,131.0,129.4,128.9,128.3,127.4,127.3,126.9,113.5,102.1,86.5$, 84.9, 82.7, 67.3, 66.0, 55.3, 54.9, 39.6, 37.7, 31.7, 30.7, 18.2, 15.8, 7.9, 7.7; IR (thin film) 1782, 1714, 1694, $1615,1518,1212,1103 \mathrm{~cm}^{-1} ;[\alpha]^{23}-65.5\left(c 1.46, \mathrm{CHCl}_{3}\right)$; HRMS (ESI) $m / z$ calcd for $\mathrm{C}_{37} \mathrm{H}_{43} \mathrm{NO}_{8} \mathrm{SiNa}(\mathrm{M}+$ $\mathrm{Na})^{+}$652.2886, found 652.2902. Anal. Calcd for $\mathrm{C}_{37} \mathrm{H}_{43} \mathrm{NO}_{8}: \mathrm{C}, 70.57 ; \mathrm{H}, 6.88 ; \mathrm{N}, 2.22$. Found: C, 70.38; H, $6.86 ; \mathrm{N}, 2.25$. 


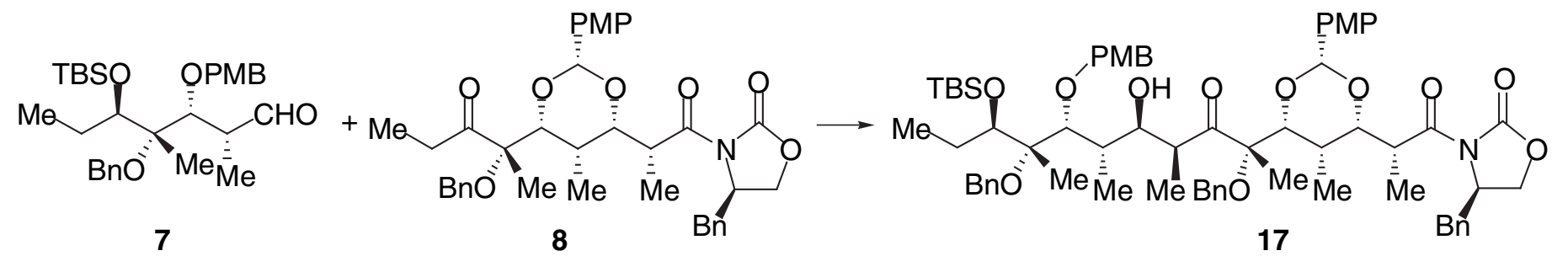

(4R)-4-Benzyl-3-[(2R)-2-[(2R,4S,5S,6R)-6-[(1S,3S,4R,5S,6R,7S,8R)-8-(tert-butyldimethylsiloxy)-1,7dibenzyloxy-4-hydroxy-6-( $p$-methoxybenzyloxy)-2-oxo-1,3,5,7-tetramethyl-decyl]-2-(p-methoxyphenyl)-5methyl-1,3-dioxan-4-yl]propionyl]-2-oxazolidinone (17). To a stirred suspension of $\operatorname{Sn}(\mathrm{OTf})_{2}(0.459 \mathrm{~g}, 1.10$ $\mathrm{mmol})$ in $5 \mathrm{~mL}$ of $\mathrm{CH}_{2} \mathrm{Cl}_{2}$ was added $\mathrm{Et}_{3} \mathrm{~N}(0.145 \mathrm{~mL}, 1.90 \mathrm{mmol})$ at $23{ }^{\circ} \mathrm{C}$. The mixture was then cooled to 0 ${ }^{\circ} \mathrm{C}$, and a solution of ketone $8(0.346 \mathrm{~g}, 0.550 \mathrm{mmol})$ in $5 \mathrm{~mL} \mathrm{of} \mathrm{CH}_{2} \mathrm{Cl}_{2}$ was added. After $4 \mathrm{~h}$, the reaction mixture was cooled to $-35^{\circ} \mathrm{C}$ followed by the addition of a solution of aldehyde $7(1.13 \mathrm{~g}, 2.20 \mathrm{mmol}) \mathrm{in} 10 \mathrm{~mL}$ of $\mathrm{CH}_{2} \mathrm{Cl}_{2}$. The mixture was maintained at $-35{ }^{\circ} \mathrm{C}$ for $40 \mathrm{~h}$, at which point $30 \mathrm{~mL}$ of saturated aqueous $\mathrm{NaHSO}_{4}$ solution was added. The mixture was extracted with $3 \times 50 \mathrm{~mL}$ of $\mathrm{CH}_{2} \mathrm{Cl}_{2}$. The combined organic layers were washed with brine $(30 \mathrm{~mL})$, dried $\left(\mathrm{MgSO}_{4}\right)$, and concentrated in vacuo. Purification by flash chromatography (5:95 to 15:85 EtOAc/hexanes) afforded the unreacted aldehyde $7(0.814 \mathrm{~g})$ and a foam $17(0.563 \mathrm{~g}, 90 \%)$ as a single stereoisomer as determined by ${ }^{1} \mathrm{H}$ NMR spectroscopy of the crude mixture: ${ }^{1} \mathrm{H} \mathrm{NMR}\left(\mathrm{CDCl}_{3}, 500 \mathrm{MHz}\right)$ $\delta 7.42-7.23(\mathrm{~m}, 19 \mathrm{H}), 6.86(\mathrm{~m}, 4 \mathrm{H}), 5.47(\mathrm{~s}, 1 \mathrm{H}), 4.88(\mathrm{~d}, J=11.8 \mathrm{~Hz}, 1 \mathrm{H}), 4.72(\mathrm{~m}, 5 \mathrm{H}), 4.39(\mathrm{~d}, J=11.3 \mathrm{~Hz}$, $1 \mathrm{H}), 4.30(\mathrm{~d}, 1 \mathrm{H}), 4.24(\mathrm{~m}, 2 \mathrm{H}), 4.12(\mathrm{~m}, 2 \mathrm{H}), 4.01(\mathrm{~s}, 1 \mathrm{H}), 3.86(\mathrm{~d}, J=10.1 \mathrm{~Hz}, 1 \mathrm{H}), 3.81(\mathrm{~s}, 3 \mathrm{H}), 3.73(\mathrm{~s}, 3 \mathrm{H})$, $3.66(\mathrm{dd}, J=7.4 \mathrm{~Hz}, 1 \mathrm{H}), 3.43(\mathrm{q}, J=6.9 \mathrm{~Hz}, 1 \mathrm{H}), 3.27(\mathrm{dd}, J=13.4,3.2 \mathrm{~Hz}, 1 \mathrm{H}), 3.00(\mathrm{~d}, 1 \mathrm{H}), 2.82(\mathrm{dd}, J=$ 13.4, $10.4 \mathrm{~Hz}, 1 \mathrm{H}), 2.06(\mathrm{~m}, 1 \mathrm{H}), 1.82(\mathrm{~m}, 1 \mathrm{H}), 1.67(\mathrm{~m}, 2 \mathrm{H}), 1.54(\mathrm{~s}, 3 \mathrm{H}), 1.41(\mathrm{~d}, J=5.8 \mathrm{~Hz}, 3 \mathrm{H}), 1.37(\mathrm{~s}$, $3 \mathrm{H}), 1.15(\mathrm{~d}, J=6.9 \mathrm{~Hz}, 3 \mathrm{H}), 1.03(\mathrm{~m}, 6 \mathrm{H}), 1.00(\mathrm{~s}, 9 \mathrm{H}), 0.70(\mathrm{~d}, J=6.8 \mathrm{~Hz}, 3 \mathrm{H}), 0.14(\mathrm{~s}, 3 \mathrm{H}), 0.13(\mathrm{~s}, 3 \mathrm{H}) ;{ }^{13} \mathrm{C}$ $\operatorname{NMR}\left(\mathrm{CDCl}_{3}, 125 \mathrm{MHz}\right) \delta 219.3,174.6,160.0,158.6,152.5,140.7,138.4,134.9,132.4,130.5,129.4,128.93$, $128.85,128.3,128.0,127.5,127.4,127.3,126.9,126.6,113.6,113.4,102.5,87.0,85.5,84.5,82.7,81.5,79.1$, 75.0, 71.4, 67.1, 66.0, 65.7, 55.2, 55.1, 54.9, 42.0, 39.5, 37.7, 36.9, 30.6, 26.2, 25.2, 19.2, 18.4, 16.9, 15.8, 11.7, 10.4, 8.5, 7.8, -3.7, -3.8; IR (thin film) 3547, 1784, 1736, 1694, 1614, 1515, 1248, $1054 \mathrm{~cm}^{-1} ;[\alpha]^{23}{ }_{\mathrm{D}}-22.3(c$ 1.55, $\mathrm{CHCl}_{3}$ ); Anal. Calcd for $\mathrm{C}_{67} \mathrm{H}_{89} \mathrm{NO}_{13} \mathrm{Si}: \mathrm{C}, 70.31 ; \mathrm{H}, 7.84 ; \mathrm{N}, 1.22$. Found: $\mathrm{C}, 70.03 ; \mathrm{H}, 7.94 ; \mathrm{N}, 1.24$.

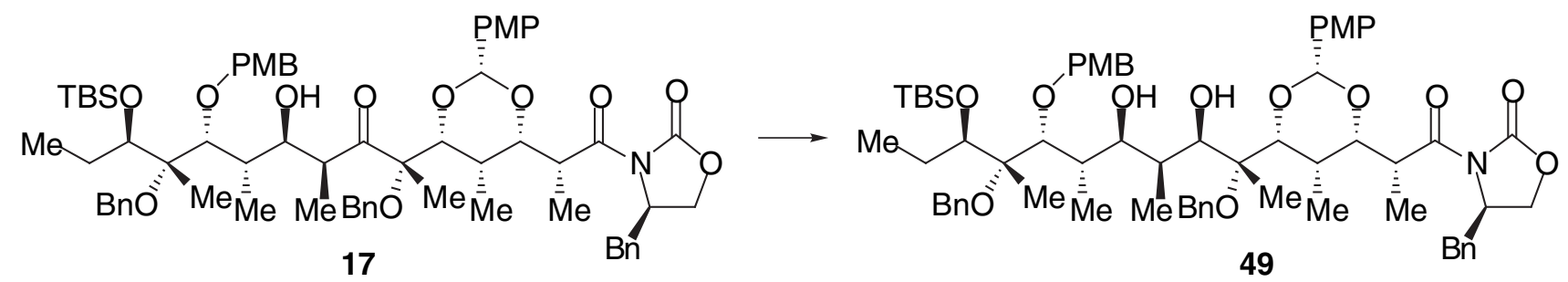

(4R)-4-Benzyl-3-[(2R)-2-[(2R,4S,5S,6R)-6-[(1S,2R,3S,4R,5S,6R,7S,8R)-8-(tert-butyldimethylsiloxy)-1,7dibenzyloxy-2,4-dihydroxy-6-( $p$-methoxybenzyloxy)-1,3,5,7-tetramethyldecyl]-2-( $p$-methoxyphenyl)-5methyl-1,3-dioxan-4-yl]propionyl]-2-oxazolidinone (49). To a solution of aldol adduct 17 (0.115 g, 0.100 $\mathrm{mmol})$ in $2 \mathrm{~mL}$ of $\mathrm{CH}_{2} \mathrm{Cl}_{2}$ at $0{ }^{\circ} \mathrm{C}$ was added $1.50 \mathrm{~mL}\left(0.300 \mathrm{mmol}, 0.20 \mathrm{M}\right.$ in $\left.\mathrm{Et}_{2} \mathrm{O}\right)$ of $\mathrm{Zn}\left(\mathrm{BH}_{4}\right)_{2}$. After $4 \mathrm{~h}, 1$ $\mathrm{mL}$ each of $\mathrm{pH} 7$ buffer and $\mathrm{MeOH}$ was added slowly, and the resultant mixture was warmed to $23{ }^{\circ} \mathrm{C}$ and stirred for $12 \mathrm{~h}$. The mixture was extracted with $3 \times 20 \mathrm{~mL} \mathrm{CH}_{2} \mathrm{Cl}_{2}$, and the combined organic extracts were dried over $\mathrm{Mg}_{2} \mathrm{SO}_{4}$, filtered, and concentrated in vacuo. The residue was dissolved in $20 \mathrm{~mL}$ of $\mathrm{MeOH}$ and stirred at $23{ }^{\circ} \mathrm{C}$ for $24 \mathrm{~h}$. Following concentration, the residue was partitioned between $10 \mathrm{~mL}$ of saturated 
aqueous $\mathrm{NH}_{4} \mathrm{Cl}$ and $10 \mathrm{~mL}$ of $\mathrm{CH}_{2} \mathrm{Cl}_{2}$. The aqueous layer was extracted with $3 \times 20 \mathrm{~mL} \mathrm{CH}_{2} \mathrm{Cl}_{2}$. The combined organic layers were washed with brine $(10 \mathrm{~mL})$, dried $\left(\mathrm{MgSO}_{4}\right)$, and concentrated in vacuo. Purification by flash chromatography (2:98 EtOAc/benzene) afforded 49 as a foam $(0.094 \mathrm{~g}, 82 \%):{ }^{1} \mathrm{H} \mathrm{NMR}\left(\mathrm{CDCl}_{3}, 500\right.$ $\mathrm{MHz}) \delta 7.50(\mathrm{~d}, J=8.7 \mathrm{~Hz}, 2 \mathrm{H}), 7.36(\mathrm{~m}, 13 \mathrm{H}), 7.24(\mathrm{~m}, 4 \mathrm{H}), 6.98(\mathrm{~d}, J=8.6 \mathrm{~Hz}, 2 \mathrm{H}), 6.79(\mathrm{~d}, J=8.5 \mathrm{~Hz}$, 2H), $5.65(\mathrm{~s}, 1 \mathrm{H}), 4.94(\mathrm{~d}, J=11.5 \mathrm{~Hz}, 1 \mathrm{H}), 4.75(\mathrm{~m}, 4 \mathrm{H}), 4.63(\mathrm{~d}, J=11.5 \mathrm{~Hz}, 1 \mathrm{H}), 4.59(\mathrm{~m}, 1 \mathrm{H}), 4.28(\mathrm{~s}, 1 \mathrm{H})$, 4.22-4.07 (m, 4H), $4.02(\mathrm{~m}, 1 \mathrm{H}), 3.94(\mathrm{t}, J=8.4 \mathrm{~Hz}, 1 \mathrm{H}), 3.89(\mathrm{~s}, 3 \mathrm{H}), 3.80(\mathrm{~m}, 1 \mathrm{H}$ and s, 3H), $3.53(\mathrm{~d}, J=9.7$ $\mathrm{Hz}, 1 \mathrm{H}), 3.36(\mathrm{br}, 1 \mathrm{H}), 3.24(\mathrm{dd}, J=13.3,3.1 \mathrm{~Hz}, 1 \mathrm{H}), 3.12(\mathrm{br}, 1 \mathrm{H}), 2.76(\mathrm{dd}, J=13.3,9.6 \mathrm{~Hz}, 1 \mathrm{H}), 2.12(\mathrm{~m}$, $1 \mathrm{H}), 1.96(\mathrm{~m}, 1 \mathrm{H}), 1.91(\mathrm{~m}, 1 \mathrm{H}), 1.84(\mathrm{~m}, 1 \mathrm{H}), 1.72(\mathrm{~m}, 1 \mathrm{H}), 1.50(\mathrm{~s}, 3 \mathrm{H}), 1.46(\mathrm{~s}, 3 \mathrm{H}), 1.44(\mathrm{~d}, J=6.5 \mathrm{~Hz}$, $3 \mathrm{H}), 1.21(\mathrm{~d}, J=6.9 \mathrm{~Hz}, 3 \mathrm{H}), 1.08(\mathrm{t}, J=7.5 \mathrm{~Hz}, 3 \mathrm{H}), 1.03(\mathrm{~s}, 9 \mathrm{H}), 1.01(\mathrm{~d}, J=6.9 \mathrm{~Hz}, 3 \mathrm{H}), 0.99(\mathrm{~d}, J=6.9$ $\mathrm{Hz}, 3 \mathrm{H}), 0.18$ (s, 3H), $0.17(\mathrm{~s}, 3 \mathrm{H}) ;{ }^{13} \mathrm{C} \mathrm{NMR}\left(\mathrm{CDCl}_{3}, 125 \mathrm{MHz}\right) \delta 174.4,159.9,158.8,152.6,140.1,139.6$, 135.0, 131.9, 131.1, 129.4, 129.0, 128.9, 128.22, 128.16, 127.6, 127.4, 127.3, 127.2, 127.1, 127.0, 113.6, 113.4, $102.1,85.4,84.4,82.9,82.2,81.3,80.7,78.8,78.3,74.9,66.3,66.0,65.9,55.3,55.2,55.0,39.5,37.6,36.7$, $33.7,30.9,26.2,25.6,18.4,18.2,17.8,15.8,11.5,11.0,8.5,6.1,-3.7,-3.8$; IR (thin film) $3502,1784,1695$, $1614,1515,1249,1077 \mathrm{~cm}^{-1} ;[\alpha]^{23}{ }_{D}-33.7\left(c 0.70, \mathrm{CHCl}_{3}\right)$; Anal. Calcd for $\mathrm{C}_{67} \mathrm{H}_{91} \mathrm{NO}_{13} \mathrm{Si}: \mathrm{C}, 70.19 ; \mathrm{H}, 8.00 ; \mathrm{N}$, 1.22. Found: C, 70.27; H, 7.96; N, 1.33 .

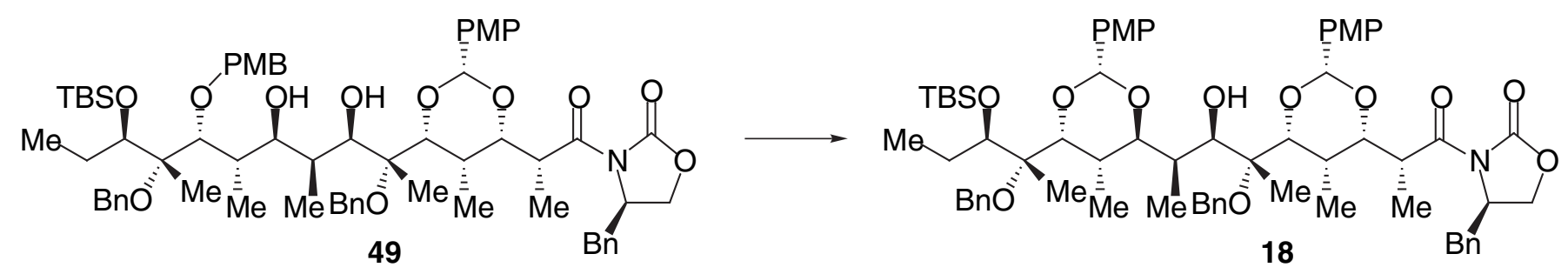

(4R)-4-Benzyl-3-[(2R)-2-[(2R,4S,5S,6R)-6-[(1S,2R,3R)-3-[(2R,4S,5S,6R)-6-[(1S,2R)-1-benzyloxy-2-(tertbutyldimethylsiloxy)-1-methyl-butyl]-2-(p-methoxyphenyl)-5-methyl-1,3-dioxan-4-yl]-1-benzyloxy-2hydroxy-1-methylbutyl]-2-(p-methoxyphenyl)-5-methyl-1,3-dioxan-4-yl]propionyl]-2-oxazolidinone (18). To a mixture of $49(0.126 \mathrm{~g}, 0.110 \mathrm{mmol})$ and $0.100 \mathrm{~g}$ of activated $4 \AA$ molecular sieves powder in $3 \mathrm{~mL}$ of $\mathrm{CH}_{2} \mathrm{Cl}_{2}$ was added a solution of DDQ $(0.030 \mathrm{~g}, 0.132 \mathrm{mmol})$ in $3 \mathrm{~mL}$ of $\mathrm{CH}_{2} \mathrm{Cl}_{2}$ at $0{ }^{\circ} \mathrm{C}$ by syringe. After $1 \mathrm{~h}$, the mixture was filtered through Celite, and the filtrate was washed with $10 \mathrm{~mL}$ of saturated aqueous $\mathrm{NaHCO}_{3}$ solution. The aqueous layer was back-extracted with $2 \times 20 \mathrm{~mL}$ of $\mathrm{CH}_{2} \mathrm{Cl}_{2}$. The combined organic layers were washed with brine $(10 \mathrm{~mL})$, dried $\left(\mathrm{MgSO}_{4}\right)$, and concentrated in vacuo. Purification by flash chromatography (15:85 EtOAc/hexanes) afforded 18 as a foam $(0.112 \mathrm{~g}, 89 \%):{ }^{1} \mathrm{H}$ NMR $\left(\mathrm{CDCl}_{3}, 500 \mathrm{MHz}\right) \delta 7.59(\mathrm{~d}, J=8.7$ $\mathrm{Hz}, 2 \mathrm{H}), 7.52(\mathrm{~d}, J=8.7 \mathrm{~Hz}, 2 \mathrm{H}), 7.46-7.28(\mathrm{~m}, 15 \mathrm{H}), 7.00(\mathrm{~m}, 4 \mathrm{H}), 5.78(\mathrm{~s}, 1 \mathrm{H}), 5.71(\mathrm{~s}, 1 \mathrm{H}), 5.19(\mathrm{~d}, J=$ $11.9 \mathrm{~Hz}, 1 \mathrm{H}), 4.85(\mathrm{~d}, J=11.2 \mathrm{~Hz}, 1 \mathrm{H}), 4.73(\mathrm{~m}, 1 \mathrm{H}), 4.66(\mathrm{~d}, J=11.2 \mathrm{~Hz}, 1 \mathrm{H}), 4.61(\mathrm{~d}, J=11.9 \mathrm{~Hz}, 1 \mathrm{H}), 4.48$ $(\mathrm{d}, J=1.6 \mathrm{~Hz}, 1 \mathrm{H}), 4.44(\mathrm{~d}, J=4.6 \mathrm{~Hz}, 1 \mathrm{H}), 4.22(\mathrm{~m}, 5 \mathrm{H}), 3.94(\mathrm{~d}, J=7.2 \mathrm{~Hz}, 1 \mathrm{H}), 3.90(\mathrm{~s}, 6 \mathrm{H}), 3.76(\mathrm{~d}, J=$ $10.0 \mathrm{~Hz}, 1 \mathrm{H}), 3.41(\mathrm{~d}, J=7.2 \mathrm{~Hz}, 1 \mathrm{H}), 3.31(\mathrm{dd}, J=13.3,2.8 \mathrm{~Hz}, 1 \mathrm{H}), 2.84(\mathrm{dd}, J=13.3,9.6 \mathrm{~Hz}, 1 \mathrm{H}), 2.54(\mathrm{~m}$, $1 \mathrm{H}), 2.06(\mathrm{~m}, 3 \mathrm{H}), 1.63(\mathrm{~d}, J=6.8 \mathrm{~Hz}, 3 \mathrm{H}), 1.55(\mathrm{~m}, 1 \mathrm{H}), 1.49(\mathrm{~d}, J=6.3 \mathrm{~Hz}, 3 \mathrm{H}), 1.40(\mathrm{~s}, 3 \mathrm{H}), 1.28(\mathrm{~d}, J=7.0$ $\mathrm{Hz}, 3 \mathrm{H}), 1.27$ (d, J=7.3 Hz, 3H), 1.24 (s, 3H), 1.16 (t, J=7.5 Hz, 3H), 1.12 (s, 9H), 0.25 (s, 3H), 0.23 (s, 3H); ${ }^{13} \mathrm{C} \mathrm{NMR}\left(\mathrm{CDCl}_{3}, 125 \mathrm{MHz}\right) \delta 174.1,160.0,159.8,152.6,139.9,139.1,135.0,131.8,130.5,129.4,128.9$, 128.2 , 128.1, 127.4, 127.3, 127.2, 127.01, 126.98, 113.7, 102.0, 96.5, 84.3, 83.8, 83.4, 81.5, 79.7, 76.3, 75.0, 73.2, 66.2, 66.0, 64.0, 55.2, 55.0, 39.4, 37.6, 33.5, 31.3, 30.0, 26.0, 24.2, 18.5, 18.4, 17.1, 15.8, 15.0, 12.2, 10.3, 9.0, -3.4, -4.3; IR (thin film) 3538, 1789, 1738, 1693, 1588, 1510, $1061 \mathrm{~cm}^{-1} ;[\alpha]_{\mathrm{D}}^{23}-25.5\left(c 1.06, \mathrm{CHCl}_{3}\right)$; Anal. Calcd for $\mathrm{C}_{67} \mathrm{H}_{89} \mathrm{NO}_{13} \mathrm{Si}: \mathrm{C}, 70.31 ; \mathrm{H}, 7.84 ; \mathrm{N}, 1.22$. Found: C, 70.09; H, 8.11; N, 1.20. 


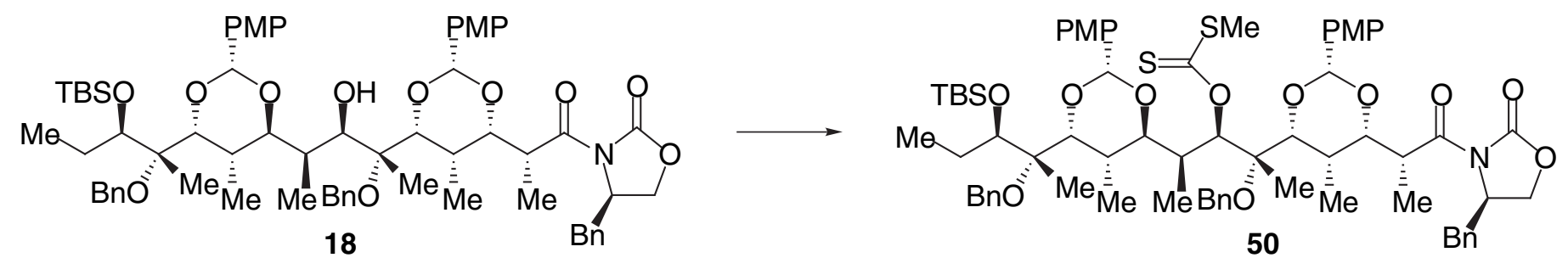

(4R)-4-Benzyl-3-[(2R)-2-[(2R,4S,5S,6R)-6-[(1S,2R,3R)-3-[(2R,4S,5S,6R)-6-[(1S,2R)-1-benzyloxy-2-(tertbutyldimethylsiloxy)-1-methyl-butyl]-2-(4-methoxyphenyl)-5-methyl-1,3-dioxan-4-yl]-1-benzyloxy-2hydroxy-1-methylbutyl]-2-(4-methoxyphenyl)-5-methyl-1,3-dioxan-4-yl]propionyl]-2-oxazolidinone, methyl dithiocarbonate (50). ${ }^{6}$ To a suspension of $\mathrm{NaH}(0.023 \mathrm{~g}, 60 \%$ oil dispersion, $0.578 \mathrm{mmol}$, washed with $3 \times 5 \mathrm{~mL}$ of hexanes) in $4 \mathrm{~mL}$ of THF was added a solution of alcohol $18(0.330 \mathrm{~g}, 0.289 \mathrm{mmol})$ in $4 \mathrm{~mL}$ of $\mathrm{THF}$ at $0{ }^{\circ} \mathrm{C}$, followed by freshly distilled $\mathrm{CS}_{2}(0.174 \mathrm{~mL}, 2.89 \mathrm{mmol})$. The resultant mixture was stirred for $1 \mathrm{~h}$ at $0{ }^{\circ} \mathrm{C}$ and $1 \mathrm{~h}$ at $23{ }^{\circ} \mathrm{C}$. The reaction mixture was then cooled to $0{ }^{\circ} \mathrm{C}$ before $0.720 \mathrm{~mL}(11.6 \mathrm{mmol})$ of methyl iodide was added by syringe. After $4 \mathrm{~h}$ at $0{ }^{\circ} \mathrm{C}$, the reaction mixture was warmed to $23{ }^{\circ} \mathrm{C}$ and allowed to stir for $12 \mathrm{~h}$. The mixture was partitioned between $10 \mathrm{~mL}$ of $\mathrm{CH}_{2} \mathrm{Cl}_{2}$ and $5 \mathrm{~mL}$ of $\mathrm{H}_{2} \mathrm{O}$, and the aqueous layer was extracted with $3 \times 20 \mathrm{~mL}$ of $\mathrm{CH}_{2} \mathrm{Cl}_{2}$. The combined organic layers were washed with brine $(10 \mathrm{~mL})$, dried $\left(\mathrm{MgSO}_{4}\right)$, and concentrated in vacuo. Purification by flash chromatography (10:90 to 15:85 EtOAc/hexanes) afforded 50 as a foam $(0.310 \mathrm{~g}, 87 \%):{ }^{1} \mathrm{H} \mathrm{NMR}\left(\mathrm{CDCl}_{3}, 500 \mathrm{MHz}\right) \delta 7.49(\mathrm{~d}, J=8.7 \mathrm{~Hz}, 2 \mathrm{H}), 7.44(\mathrm{~d}, J=8.7$ $\mathrm{Hz}, 2 \mathrm{H}), 7.42-7.26(\mathrm{~m}, 15 \mathrm{H}), 6.95(\mathrm{~d}, J=8.7 \mathrm{~Hz}, 2 \mathrm{H}), 6.92(\mathrm{~d}, J=8.7 \mathrm{~Hz}, 2 \mathrm{H}), 6.30(\mathrm{~s}, 1 \mathrm{H}), 5.55(\mathrm{~s}, 1 \mathrm{H}), 5.48$ $(\mathrm{s}, 1 \mathrm{H}), 5.02(\mathrm{~d}, J=12.1 \mathrm{~Hz}, 1 \mathrm{H}), 4.93(\mathrm{~d}, J=11.8 \mathrm{~Hz}, 1 \mathrm{H}), 4.74(\mathrm{~m}, 1 \mathrm{H}), 4.70(\mathrm{~d}, J=11.8 \mathrm{~Hz}, 1 \mathrm{H}), 4.57(\mathrm{~d}, J$ $=12.1 \mathrm{~Hz}, 1 \mathrm{H}), 4.25(\mathrm{~m}, 3 \mathrm{H}), 4.14(\mathrm{~m}, 2 \mathrm{H}), 4.03(\mathrm{~d}, J=4.5 \mathrm{~Hz}, 1 \mathrm{H}), 3.92(\mathrm{~d}, J=1.4 \mathrm{~Hz}, 1 \mathrm{H}), 3.88(\mathrm{~s}, 3 \mathrm{H})$, $3.86(\mathrm{~s}, 3 \mathrm{H}), 3.74(\mathrm{~d}, J=11.1 \mathrm{~Hz}, 1 \mathrm{H}), 3.30(\mathrm{dd}, J=13.4,3.0 \mathrm{~Hz}, 1 \mathrm{H}), 3.13(\mathrm{~m}, 1 \mathrm{H}), 2.83(\mathrm{dd}, J=13.4,9.6 \mathrm{~Hz}$, $1 \mathrm{H}), 2.47(\mathrm{~s}, 3 \mathrm{H}), 2.13(\mathrm{~m}, 1 \mathrm{H}), 2.06(\mathrm{~m}, 1 \mathrm{H}), 1.90(\mathrm{~m}, 1 \mathrm{H}), 1.55(\mathrm{~m}, 1 \mathrm{H}), 1.51(\mathrm{~s}, 3 \mathrm{H}), 1.48(\mathrm{~d}, J=6.7 \mathrm{~Hz}$, $3 \mathrm{H}), 1.43(\mathrm{~d}, J=5.9 \mathrm{~Hz}, 3 \mathrm{H}), 1.31(\mathrm{~d}, J=6.7 \mathrm{~Hz}, 3 \mathrm{H}), 1.21(\mathrm{~s}, 3 \mathrm{H}), 1.16(\mathrm{~d}, J=6.9 \mathrm{~Hz}, 3 \mathrm{H}), 1.06(\mathrm{t}, J=7.5$ $\mathrm{Hz}, 3 \mathrm{H}), 1.01(\mathrm{~s}, 9 \mathrm{H}), 0.16(\mathrm{~s}, 3 \mathrm{H}), 0.12(\mathrm{~s}, 3 \mathrm{H}) ;{ }^{13} \mathrm{C}$ NMR $\left(\mathrm{CDCl}_{3}, 125 \mathrm{MHz}\right) \delta 216.4,174.4,160.0,159.8$, $152.6,140.0,138.9,135.0,131.8,131.1,129.4,129.0,128.11,128.05,127.7,127.4,127.3,127.03,127.00$, $126.9,126.7,113.6,113.4,102.5,96.2,83.8,83.0,82.0,81.7,80.7,78.1,75.4,66.12,66.06,65.9,55.3,55.2$, 55.1, 39.5, 37.7, 34.1, 31.2, 30.2, 26.1, 25.1, 18.8, 18.6, 18.4, 16.8, 15.8, 15.0, 11.8, 11.1, 8.3, -3.6, -4.0; IR (thin film) $1789,1694,1614,1588,1516,1116 \mathrm{~cm}^{-1} ;[\alpha]_{\mathrm{D}}^{23}-41.0\left(c\right.$ 1.45, $\left.\mathrm{CHCl}_{3}\right)$; Anal. Calcd for $\mathrm{C}_{69} \mathrm{H}_{91} \mathrm{NO}_{13} \mathrm{~S}_{2} \mathrm{Si}: \mathrm{C}, 67.12 ; \mathrm{H}, 7.43 ; \mathrm{N}, 1.13$. Found: C, 67.12; H, 7.50; N, 1.12.

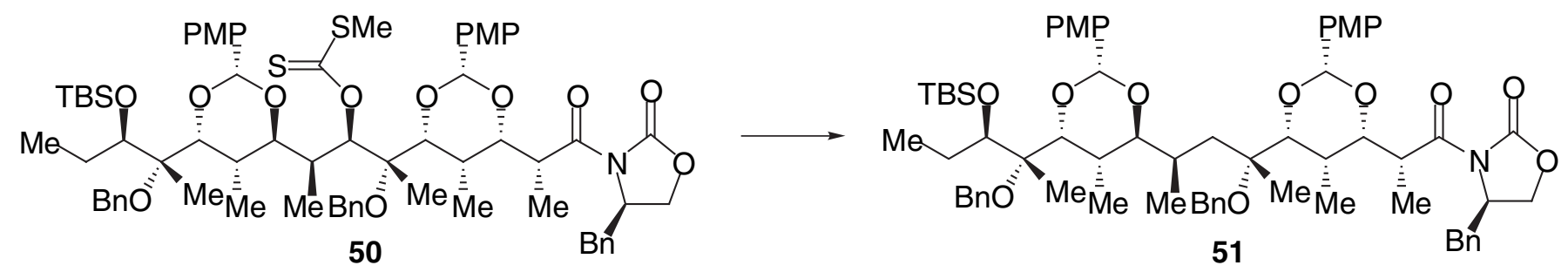

(4R)-4-Benzyl-3-[(2R)-2-[(2R,4S,5S,6R)-6-[(1S,3R)-3-[(2R,4S,5S,6R)-6-[(1S,2R)-1-benzyloxy-2-(tertbutyldimethylsiloxy)-1-methylbutyl]-2-(p-methoxyphenyl)-5-methyl-1,3-dioxan-4-yl]-1-benzyloxy-1-

\footnotetext{
${ }^{6}$ (a) Barton, D. H. R.; McCombie S. W. J. Chem. Soc., Perkin Trans. 1 1975, 1574-1585. (b) Evans, D. A.; Kim, A. S.; Metternich, R.; Novack, V. J. J. Am. Chem. Soc. 1998, 120, 5921-5942.
} 
methylbutyl]-2-(p-methoxyphenyl)-5-methyl-1,3-dioxan-4-yl]propionyl]-2-oxazolidinone (51). ${ }^{6} \quad$ Xanthate $50(0.300 \mathrm{~g}, 0.243 \mathrm{mmol})$ and a catalytic amount of AIBN $(0.002 \mathrm{~g}, 0.0141 \mathrm{mmol})$ were taken up in $3.00 \mathrm{~mL}$ of tributyltin hydride and heated to $110{ }^{\circ} \mathrm{C}$. After $30 \mathrm{~min}$, the reaction mixture was cooled to $23{ }^{\circ} \mathrm{C}$ and filtered through a plug of silica gel to remove tributyltin hydride (washed with 10:90 to 50:50 EtOAc/hexanes). Further purification by flash chromatography (1:99 EtOAc/benzene) afforded $\mathbf{5 1}$ as a foam $(0.202 \mathrm{~g}, 74 \%)$ : ${ }^{1} \mathrm{H} \mathrm{NMR}$ $\left(\mathrm{CDCl}_{3}, 500 \mathrm{MHz}\right) \delta 7.52(\mathrm{~d}, J=8.7 \mathrm{~Hz}, 2 \mathrm{H}), 7.51-7.36(\mathrm{~m}, 17 \mathrm{H}), 6.99(\mathrm{~d}, J=8.8 \mathrm{~Hz}, 2 \mathrm{H}), 6.95(\mathrm{~d}, J=8.8 \mathrm{~Hz}$, $2 \mathrm{H}), 5.65(\mathrm{~s}, 1 \mathrm{H}), 5.29(\mathrm{~s}, 1 \mathrm{H}), 5.16(\mathrm{~d}, J=11.7 \mathrm{~Hz}, 1 \mathrm{H}), 4.78(\mathrm{~m}, 3 \mathrm{H}), 4.53(\mathrm{~d}, J=11.8 \mathrm{~Hz}, 1 \mathrm{H}), 4.48(\mathrm{~d}, J=$ $2.1 \mathrm{~Hz}, 1 \mathrm{H}), 4.40(\mathrm{~m}, 1 \mathrm{H}), 4.30(\mathrm{t}, J=8.4 \mathrm{~Hz}, 1 \mathrm{H}), 4.25(\mathrm{dd}, J=9.1,2.7 \mathrm{~Hz}, 1 \mathrm{H}), 4.14(\mathrm{~m}, 2 \mathrm{H}), 3.88(\mathrm{~s}, 6 \mathrm{H}$ and $\mathrm{m}, 1 \mathrm{H}), 3.37(\mathrm{~d}, J=10.5 \mathrm{~Hz}, 1 \mathrm{H}), 3.31(\mathrm{dd}, J=13.3,3.2 \mathrm{~Hz}, 1 \mathrm{H}), 2.82(\mathrm{dd}, J=13.3,9.5 \mathrm{~Hz}, 1 \mathrm{H}), 2.49(\mathrm{~m}, 1 \mathrm{H})$, $2.20(\mathrm{~m}, 1 \mathrm{H}), 1.98(\mathrm{~m}, 2 \mathrm{H}), 1.90(\mathrm{~m}, 1 \mathrm{H}), 1.59(\mathrm{~d}, J=7.0 \mathrm{~Hz}, 3 \mathrm{H}), 1.48(\mathrm{~m}, 2 \mathrm{H}), 1.44(\mathrm{~s}, 3 \mathrm{H}), 1.42(\mathrm{~d}, J=6.6$ $\mathrm{Hz}, 3 \mathrm{H}), 1.21(\mathrm{~d}, J=6.5 \mathrm{~Hz}, 3 \mathrm{H}), 1.16(\mathrm{~d}, J=6.9 \mathrm{~Hz}, 3 \mathrm{H}), 1.08(\mathrm{t}, J=7.6 \mathrm{~Hz}, 3 \mathrm{H}), 1.06(\mathrm{~s}, 3 \mathrm{H}), 1.05(\mathrm{~s}, 9 \mathrm{H})$, $0.17(\mathrm{~s}, 3 \mathrm{H}), 0.16(\mathrm{~s}, 3 \mathrm{H}) ;{ }^{13} \mathrm{C} \mathrm{NMR}\left(\mathrm{CDCl}_{3}, 125 \mathrm{MHz}\right) \delta 174.5,159.84,159.82,152.6,140.5,140.1,135.1$, 132.0, 131.3, 129.4, 129.0, 128.2, 128.0, 127.43, 127.37, 127.3, 127.25, 126.94, 126.9, 126.7, 113.7, 113.5, $101.8,96.4,86.1,85.6,82.9,81.6,79.0,76.6,73.0,66.2,66.1,65.6,55.3,55.1,41.1,39.5,37.8,31.0,29.2$, $28.3,26.2,24.1,21.0,18.5,18.4,17.1,15.9,15.2,12.2,8.1,-3.4,-4.3$; IR (thin film) 1784, 1738, 1694, 1615, 1517, $1115 \mathrm{~cm}^{-1} ;[\alpha]_{D}^{23}-27.3\left(c 0.70, \mathrm{CHCl}_{3}\right)$; Anal. Calcd for $\mathrm{C}_{67} \mathrm{H}_{89} \mathrm{NO}_{12} \mathrm{Si}: \mathrm{C}, 71.31 ; \mathrm{H}, 7.95 ; \mathrm{N}, 1.24$. Found: C, 71.11; H, 7.99; N, 1.37.

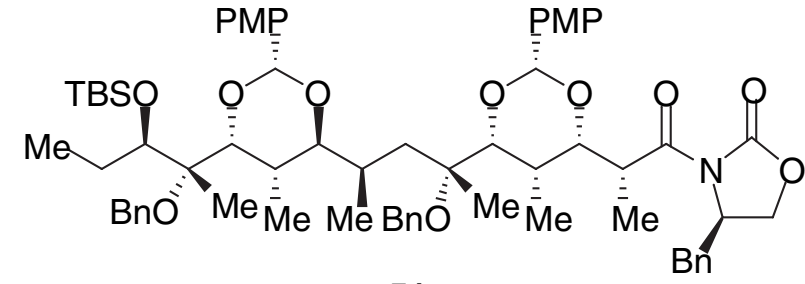

51

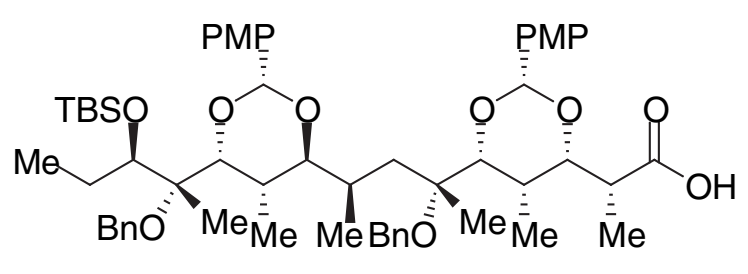

52

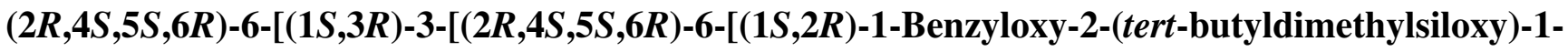
methylbutyl]-2-(p-methoxyphenyl)-5-methyl-1,3-dioxan-4-yl]-1-benzyloxy-1-methylbutyl]-4-[(1R)-1carboxyeth-1-yl]-2-(p-methoxyphenyl)-5-methyl-1,3-dioxane (52). To a solution of imide 51 (0.176 g, 0.156 $\mathrm{mmol})$ in $7.5 \mathrm{~mL}$ of THF and $1.5 \mathrm{~mL}$ of $\mathrm{H}_{2} \mathrm{O}$ at $0{ }^{\circ} \mathrm{C}$ was added $0.177 \mathrm{~mL}(1.56 \mathrm{mmol})$ of a $30 \%$ aqueous solution of $\mathrm{H}_{2} \mathrm{O}_{2}$ followed by $2.34 \mathrm{~mL}(0.468 \mathrm{mmol})$ of a $0.2 \mathrm{M}$ aqueous solution of $\mathrm{LiOH}$. After $1 \mathrm{~h}$, the solution was warmed to $23{ }^{\circ} \mathrm{C}$ and stirred for $6 \mathrm{~h}$. The reaction mixture was then cooled to $0{ }^{\circ} \mathrm{C}$ before treatment with $5 \mathrm{~mL}$ of a solution of $1.5 \mathrm{M} \mathrm{Na}_{2} \mathrm{SO}_{3}$. After $15 \mathrm{~min}$, the mixture was diluted with $10 \mathrm{~mL} \mathrm{of} \mathrm{Et}_{2} \mathrm{O}$ and then acidified to $\mathrm{pH} 1$ with $1 \mathrm{M} \mathrm{HCl}$. The aqueous layer was isolated and extracted with $3 \times 40 \mathrm{~mL}$ of $\mathrm{Et}_{2} \mathrm{O}$. The combined organic layers were washed with brine $(10 \mathrm{~mL})$, dried $\left(\mathrm{MgSO}_{4}\right)$, and concentrated in vacuo. Purification by flash chromatography (20:80 to 30:70 EtOAc/hexanes) afforded 52 as a foam (0.140 g, 93\%): ${ }^{1} \mathrm{H} \mathrm{NMR}\left(\mathrm{CDCl}_{3}, 500 \mathrm{MHz}\right) \delta 7.51(\mathrm{~d}, J=8.7 \mathrm{~Hz}, 2 \mathrm{H}), 7.43(\mathrm{~d}, J=8.7 \mathrm{~Hz}, 2 \mathrm{H}), 7.39-7.24(\mathrm{~m}, 10 \mathrm{H})$, $6.97(\mathrm{~m}, 4 \mathrm{H}), 5.65(\mathrm{~s}, 1 \mathrm{H}), 5.33(\mathrm{~s}, 1 \mathrm{H}), 5.13(\mathrm{~d}, J=11.8 \mathrm{~Hz}, 1 \mathrm{H}), 4.79(\mathrm{~d}, J=13.5 \mathrm{~Hz}, 1 \mathrm{H}), 4.77(\mathrm{~d}, J=13.5$ $\mathrm{Hz}, 1 \mathrm{H}), 4.56(\mathrm{~d}, J=11.8 \mathrm{~Hz}, 1 \mathrm{H}), 4.45(\mathrm{~d}, J=1.8 \mathrm{~Hz}, 1 \mathrm{H}), 4.36(\mathrm{~d}, J=4.2 \mathrm{~Hz}, 1 \mathrm{H}), 3.89(\mathrm{~s}, 3 \mathrm{H}), 3.88(\mathrm{~s}, 3 \mathrm{H})$, $3.87(\mathrm{~m}, 2 \mathrm{H}), 3.35(\mathrm{~d}, J=10.3 \mathrm{~Hz}, 1 \mathrm{H}), 2.88(\mathrm{~m}, 1 \mathrm{H}), 2.46(\mathrm{~m}, 1 \mathrm{H}), 2.14(\mathrm{~m}, 1 \mathrm{H}), 1.96(\mathrm{~m}, 3 \mathrm{H}), 1.56(\mathrm{~d}, J=6.9$ $\mathrm{Hz}, 3 \mathrm{H}), 1.44(\mathrm{~s}, 3 \mathrm{H}$ and $\mathrm{m}, 2 \mathrm{H}), 1.41(\mathrm{~d}, J=6.8 \mathrm{~Hz}, 3 \mathrm{H}), 1.28(\mathrm{~d}, J=6.8 \mathrm{~Hz}, 3 \mathrm{H}), 1.20(\mathrm{~d}, J=6.5 \mathrm{~Hz}, 3 \mathrm{H})$, $1.09(\mathrm{~s}, 3 \mathrm{H}), 1.08(\mathrm{t}, J=7.5 \mathrm{~Hz}, 3 \mathrm{H}), 1.01(\mathrm{~s}, 9 \mathrm{H}), 0.17(\mathrm{~s}, 3 \mathrm{H}), 0.13(\mathrm{~s}, 3 \mathrm{H}) ;{ }^{13} \mathrm{C} \mathrm{NMR}\left(\mathrm{CDCl}_{3}, 125 \mathrm{MHz}\right) \delta$ $178.5,159.9,159.8,140.3,140.0,132.0,131.1,128.2,128.1,127.32,127.29,127.2,127.0,126.9,126.8,113.7$, 113.6, 101.9, 96.3, 86.1, 85.6, 82.9, 81.8, 79.0, 76.7, 73.2, 66.2, 65.5, 55.3, 41.5, 40.9, 31.8, 29.2, 28.3, 26.2, $24.2,21.3,18.4,18.3,17.1,15.3,15.1,12.2,7.8,-3.3,-4.4$; IR (thin film) $1738,1616,1518,1115 \mathrm{~cm}^{-1} ;[\alpha]^{23}$ -19.1 ( $c$ 0.89, $\mathrm{CHCl}_{3}$ ); HRMS (ESI) $m / z$ calcd for $\mathrm{C}_{57} \mathrm{H}_{80} \mathrm{O}_{11} \mathrm{SiNa}(\mathrm{M}+\mathrm{Na})^{+}$969.5548, found 969.5562. 


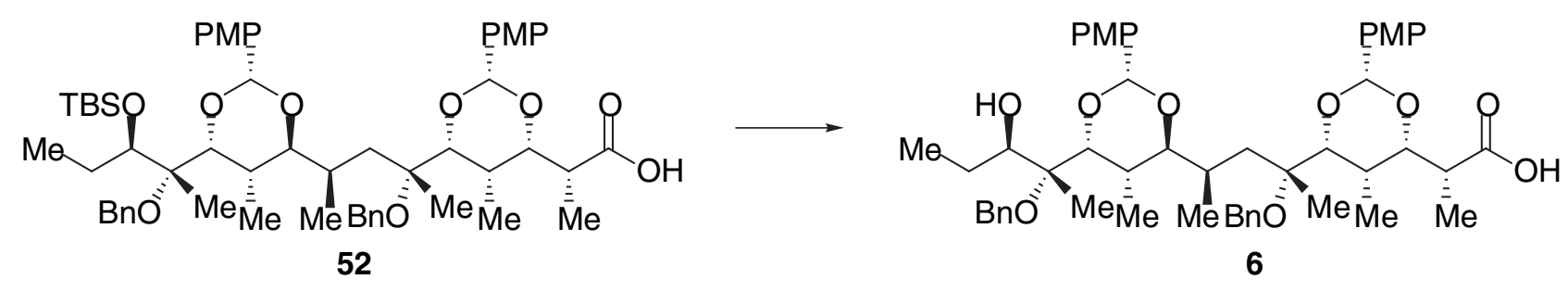

$(2 R, 4 S, 5 S, 6 R)-6$-[(1S,3R)-3-[(2R,4S,5S,6R)-6-[(1S,2R)-1-Benzyloxy-2-hydroxy-1-methylbutyl]-2- $(p-$ methoxyphenyl)-5-methyl-1,3-dioxan-4-yl]-1-benzyloxy-1-methylbutyl]-4-[(1R)-1-carboxyeth-1-yl]-2-(pmethoxyphenyl)-5-methyl-1,3-dioxane (6). To a solution of silyl ether $\mathbf{5 2}(0.136 \mathrm{~g}, 0.140 \mathrm{mmol})$ in $3 \mathrm{~mL}$ of THF at $0{ }^{\circ} \mathrm{C}$ was added $n-\mathrm{Bu}_{4} \mathrm{NF}(0.420 \mathrm{~mL}, 1.0 \mathrm{M}$ in THF, $0.420 \mathrm{mmol})$. The resultant solution was heated at reflux for $5 \mathrm{~h}$. The reaction mixture was then cooled to $23^{\circ} \mathrm{C}$, and a saturated aqueous solution of $\mathrm{NH}_{4} \mathrm{Cl}(4$ $\mathrm{mL}$ ) was added. The mixture was extracted with $3 \times 30 \mathrm{~mL}$ of EtOAc. The combined organic layers were washed with brine $(10 \mathrm{~mL})$, dried $\left(\mathrm{MgSO}_{4}\right)$, and concentrated in vacuo. Purification by flash chromatography (30:70 EtOAc/hexanes to 40:60 EtOAc/hexanes with one drop acetic acid per $100 \mathrm{~mL}$ eluant) afforded $\mathbf{6}$ as a foam $(0.110 \mathrm{~g}, 92 \%):{ }^{1} \mathrm{H} \mathrm{NMR}\left(\mathrm{CDCl}_{3}, 500 \mathrm{MHz}\right) \delta 7.51(\mathrm{~d}, J=8.7 \mathrm{~Hz}, 2 \mathrm{H}), 7.47(\mathrm{~d}, J=8.7 \mathrm{~Hz}, 2 \mathrm{H})$, $7.39-7.25(\mathrm{~m}, 10 \mathrm{H}), 6.96(\mathrm{~m}, 4 \mathrm{H}), 5.65(\mathrm{~s}, 1 \mathrm{H}), 5.59(\mathrm{~s}, 1 \mathrm{H}), 4.88(\mathrm{~d}, J=11.5 \mathrm{~Hz}, 1 \mathrm{H}), 4.74(\mathrm{~d}, J=12.1 \mathrm{~Hz}$, $1 \mathrm{H}), 4.68(\mathrm{~d}, J=12.1 \mathrm{~Hz}, 1 \mathrm{H}), 4.55(\mathrm{~d}, J=11.5 \mathrm{~Hz}, 1 \mathrm{H}), 4.26(\mathrm{~d}, J=1.9 \mathrm{~Hz}, 1 \mathrm{H}), 4.10(\mathrm{~s}, 1 \mathrm{H}), 4.09(\mathrm{~d}, J=$ $10.7 \mathrm{~Hz}, 1 \mathrm{H}), 3.95(\mathrm{~m}, 1 \mathrm{H}), 3.88(\mathrm{~s}, 3 \mathrm{H}), 3.87(\mathrm{~s}, 3 \mathrm{H}), 3.33(\mathrm{~d}, J=10.3 \mathrm{~Hz}, 1 \mathrm{H}), 2.87(\mathrm{~m}, 1 \mathrm{H}), 2.66(\mathrm{~m}, 1 \mathrm{H})$, $2.08(\mathrm{~m}, 1 \mathrm{H}), 1.96(\mathrm{~m}, 1 \mathrm{H}), 1.63(\mathrm{~m}, 1 \mathrm{H}), 1.54(\mathrm{~m}, 2 \mathrm{H}), 1.52(\mathrm{~d}, J=6.9 \mathrm{~Hz}, 3 \mathrm{H}), 1.50(\mathrm{~s}, 3 \mathrm{H}), 1.46(\mathrm{~m}, 1 \mathrm{H})$, $1.41(\mathrm{~d}, J=6.8 \mathrm{~Hz}, 3 \mathrm{H}), 1.32(\mathrm{~s}, 1 \mathrm{H}), 1.30(\mathrm{~d}, J=6.3 \mathrm{~Hz}, 3 \mathrm{H}), 1.20(\mathrm{~d}, J=6.8 \mathrm{~Hz}, 3 \mathrm{H}), 1.09(\mathrm{~s}, 3 \mathrm{H}), 1.07(\mathrm{t}, J$ $=7.4 \mathrm{~Hz}, 3 \mathrm{H}) ;{ }^{13} \mathrm{C} \mathrm{NMR}\left(\mathrm{CDCl}_{3}, 125 \mathrm{MHz}\right) \delta 177.1,160.0,159.9,140.4,139.5,131.5,131.2,128.24,128.16$, 127.4, 127.30, 127.27, 127.2, 127.1, 127.0, 113.8, 113.6, 101.8, 96.4, 87.3, 87.0, 83.5, 80.4, 79.3, 78.3, 74.6, 65.7, 65.4, 55.4, 55.3, 41.5, 40.4, 32.2, 29.1, 27.8, 24.1, 20.9, 17.7, 17.0, 16.0, 14.9, 11.4, 8.0; IR (thin film) $3438,1732,1616,1518,1103 \mathrm{~cm}^{-1} ;[\alpha]_{D}^{23}-9.8\left(c 0.61, \mathrm{CHCl}_{3}\right)$; ); HRMS (ESI) $m / z$ calcd for $\mathrm{C}_{51} \mathrm{H}_{66} \mathrm{O}_{11} \mathrm{Na}(\mathrm{M}$ $+\mathrm{Na})^{+} 877.4503$, found 877.4510 .
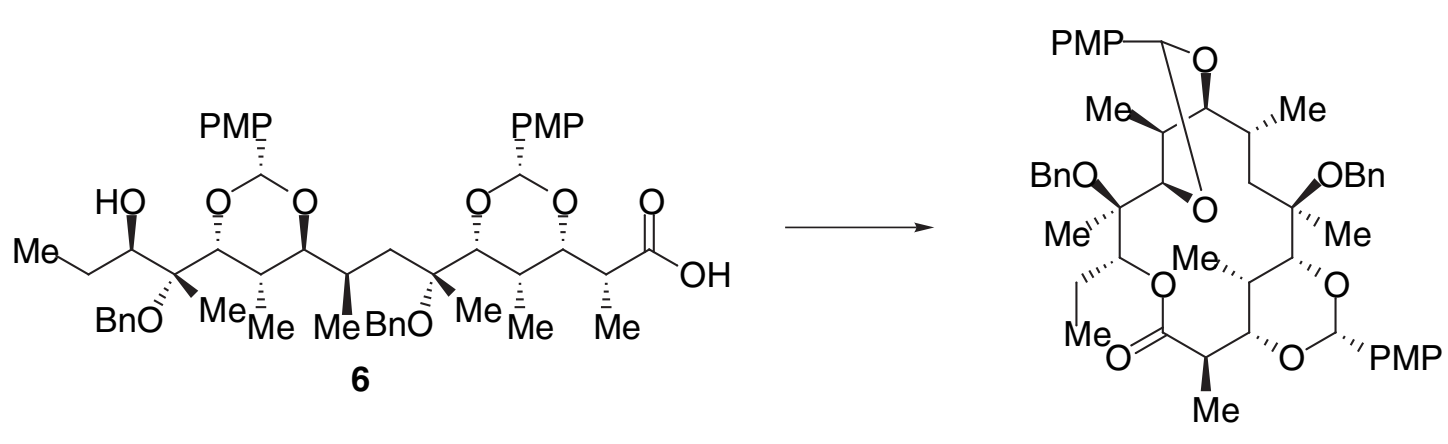

53

$(2 R, 3 S, 4 R, 5 S, 6 R, 8 R, 9 S, 10 S, 11 R, 12 S, 13 R)-6,12-D i b e n z y l o x y-13-e t h y l-3,5-[(R)$-( $p$-methoxybenzylidene)dioxy $]-9,11-[(R)$-( $p$-methoxybenzylidene $)$ dioxy $]-2,4,6,8,10,12$-hexamethyltetradecanolide (53). To a solution of azeotropically dried $(2 \times 5 \mathrm{~mL}$ of benzene) hydroxy acid $6(0.104 \mathrm{~g}, 0.122 \mathrm{mmol})$ in $12 \mathrm{~mL}$ of benzene at $23{ }^{\circ} \mathrm{C}$ was added $i$-Pr ${ }_{2} \mathrm{NEt}(0.212 \mathrm{~mL}, 1.22 \mathrm{mmol})$, followed by 2,4,6-trichlorobenzoyl chloride $(0.191 \mathrm{~mL}, 1.22 \mathrm{mmol})$. After $5 \mathrm{~h}, N, N$-(dimethylamino)pyridine $(0.596 \mathrm{~g}, 4.88 \mathrm{mmol})$ was added as well as 5 $\mathrm{mL}$ of benzene. After $1 \mathrm{~h}$, an aqueous solution of $1 \mathrm{~N} \mathrm{NaHSO}_{4}(20 \mathrm{~mL})$ was added. The mixture was extracted 
with $3 \times 40 \mathrm{~mL}$ of $\mathrm{CH}_{2} \mathrm{Cl}_{2}$. The combined organic layers were washed with brine $(10 \mathrm{~mL}), \operatorname{dried}\left(\mathrm{MgSO}_{4}\right)$, and concentrated in vaсиo. Purification by flash chromatography (10:90 to 15:85 EtOAc/hexanes) afforded $\mathbf{5 3}$ as a foam $(0.086 \mathrm{~g}, 84 \%):{ }^{1} \mathrm{H} \mathrm{NMR}\left(\mathrm{CDCl}_{3}, 500 \mathrm{MHz}\right) \delta 7.61(\mathrm{~d}, J=8.7 \mathrm{~Hz}, 2 \mathrm{H}), 7.55(\mathrm{~d}, J=8.7 \mathrm{~Hz}, 2 \mathrm{H}), 7.41-$ $7.28(\mathrm{~m}, 10 \mathrm{H}), 7.02(\mathrm{~m}, 4 \mathrm{H}), 5,85(\mathrm{dd}, J=11.0,1.5 \mathrm{~Hz}, 1 \mathrm{H}), 5.79(\mathrm{~s}, 1 \mathrm{H}), 5.75(\mathrm{~s}, 1 \mathrm{H}), 5.26(\mathrm{~d}, J=12.3 \mathrm{~Hz}$, $1 \mathrm{H}), 4.80(\mathrm{~d}, J=12.3 \mathrm{~Hz}, 1 \mathrm{H}), 4.67(\mathrm{~d}, J=12.3 \mathrm{~Hz}, 1 \mathrm{H}), 4.56(\mathrm{~d}, J=12.3 \mathrm{~Hz}, 1 \mathrm{H}), 4.35(\mathrm{~s}, 1 \mathrm{H}), 4.06(\mathrm{~d}, J=$ $10.8 \mathrm{~Hz}, 1 \mathrm{H}), 3.95(\mathrm{~s}, 1 \mathrm{H}), 3.90(\mathrm{~s}, 3 \mathrm{H}), 3.89(\mathrm{~s}, 3 \mathrm{H}), 3.43(\mathrm{~d}, J=10.7 \mathrm{~Hz}, 1 \mathrm{H}), 3.02(\mathrm{~m}, 1 \mathrm{H}), 2.64(\mathrm{~m}, 1 \mathrm{H})$, $2.13(\mathrm{~m}, 1 \mathrm{H}), 1.94(\mathrm{~m}, 1 \mathrm{H}), 1.81(\mathrm{~m}, 1 \mathrm{H}), 1.71(\mathrm{~m}, 1 \mathrm{H}), 1.61(\mathrm{~d}, J=6.6 \mathrm{~Hz}, 3 \mathrm{H}$ and $\mathrm{m}, 2 \mathrm{H}), 1.50(\mathrm{~s}, 3 \mathrm{H}), 1.42$ $(\mathrm{d}, J=6.5 \mathrm{~Hz}, 3 \mathrm{H}), 1.40(\mathrm{~d}, J=6.8 \mathrm{~Hz}, 3 \mathrm{H}), 1.29(\mathrm{~d}, J=6.3 \mathrm{~Hz}, 3 \mathrm{H}$ and s, $3 \mathrm{H}), 0.97(\mathrm{t}, J=7.4 \mathrm{~Hz}, 3 \mathrm{H}) ;{ }^{13} \mathrm{C}$ $\operatorname{NMR}\left(\mathrm{CDCl}_{3}, 125 \mathrm{MHz}\right) \delta 174.7,159.9,159.8,140.3,139.7,131.8,131.1,128.2,128.1,127.4,127.3,127.1$, 127.0, 126.8, 113.6, 113.5, 102.4, 95.8, 87.2, 86.7, 84.5, 79.4, 78.8, 77.1, 75.4, 67.3, 65.1, 55.3, 42.0, 38.8, 32.7, $28.4,27.5,21.1,20.9,17.8,17.7,14.1,10.4,8.9 ;$ IR (thin film) $1731,1616,1519,1116 \mathrm{~cm}^{-1} ;[\alpha]^{23}{ }_{\mathrm{D}}-38.8(c$ $0.58, \mathrm{CHCl}_{3}$ ); HRMS (ESI) $m / z$ calcd for $\mathrm{C}_{51} \mathrm{H}_{64} \mathrm{O}_{10} \mathrm{Na}(\mathrm{M}+\mathrm{Na})^{+} 859.4397$, found 859.4396.

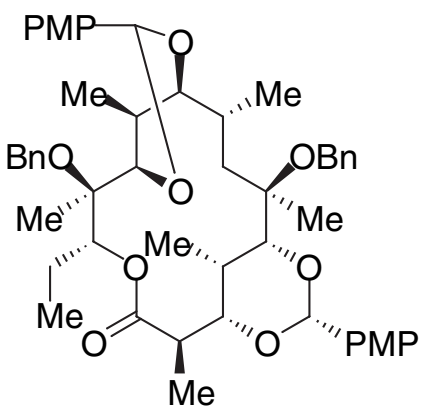

53

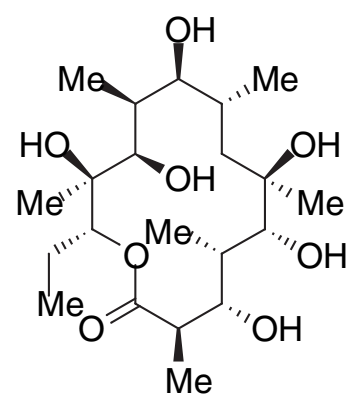

1

$(2 R, 3 S, 4 R, 5 S, 6 R, 8 R, 9 S, 10 S, 11 R, 12 S, 13 R)-13-E t h y l-3,5,6,9,11,12-h e x a h y d r o x y-2,4,6,8,10,12-$

hexamethyltetradecanolide [(9S)-Dihydroerythronolide A] (1). To a solution of lactone 53 (0.086 g, 0.103 $\mathrm{mmol}$ ) in $5 \mathrm{~mL}$ of $\mathrm{EtOH}$ at $23{ }^{\circ} \mathrm{C}$ was added $150 \mathrm{mg}$ of $\mathrm{Pd}(\mathrm{OH})_{2}$ on carbon. The flask was purged for $5 \mathrm{~min}$ with $\mathrm{H}_{2}$ under balloon pressure and then maintained under positive pressure (balloon). After $20 \mathrm{~h}$, the mixture was filtered and the filtrate was concentrated in vacuo. Purification by flash chromatography (50:50 to 70:30 EtOAc/hexanes) afforded (9S)-dihydroerythronolide A $1(0.041 \mathrm{~g}, 95 \%)$ as a white solid. Recrystallization from EtOAc-hexanes afforded fine needles: $\mathrm{mp}$ 205-206 ${ }^{\circ} \mathrm{C}$ \{lit: ${ }^{7} \mathrm{mp} 203-206{ }^{\circ} \mathrm{C}$ (acetone-hexane) $\}$; ${ }^{1} \mathrm{H}$ NMR $\left(\mathrm{CDCl}_{3}, 500 \mathrm{MHz}\right) \delta 4.63(\mathrm{dd}, J=10.7,1.6 \mathrm{~Hz}, 1 \mathrm{H}), 4.28(\mathrm{~d}, J=4.2 \mathrm{~Hz}, 1 \mathrm{H}), 3.96(\mathrm{~s}, 1 \mathrm{H}), 3.85(\mathrm{~d}, J=10.3 \mathrm{~Hz}$, $1 \mathrm{H}), 3.52$ (br, 1H), 3.49 (d, $J=9.0 \mathrm{~Hz}, 1 \mathrm{H}), 3.48(\mathrm{~s}, 1 \mathrm{H}), 3.03(\mathrm{br}, 1 \mathrm{H}), 2.96$ (td, $J=9.1,2.5 \mathrm{~Hz}, 1 \mathrm{H}), 2.79$ (dq, $J=10.4,6.7 \mathrm{~Hz}, 1 \mathrm{H}), 2.58(\mathrm{~s}, 1 \mathrm{H}), 2.05(\mathrm{~m}, 1 \mathrm{H}), 1.93(\mathrm{~m}, 1 \mathrm{H}$ and s, $1 \mathrm{H}), 1.56(\mathrm{~m}, 3 \mathrm{H}), 1.43(\mathrm{dd}, J=14.9,2.5$ $\mathrm{Hz}, 1 \mathrm{H}), 1.30(\mathrm{~d}, J=6.2 \mathrm{~Hz}, 3 \mathrm{H}), 1.29(\mathrm{~d}, J=6.7 \mathrm{~Hz}, 3 \mathrm{H}), 1.24(\mathrm{~s}, 3 \mathrm{H}), 1.23(\mathrm{~d}, J=5.9 \mathrm{~Hz}, 3 \mathrm{H}), 1.20(\mathrm{dd}, J=$ 14.9, $2.4 \mathrm{~Hz}, 1 \mathrm{H}), 1.06(\mathrm{~s}, 3 \mathrm{H}), 1.04(\mathrm{~d}, J=6.9 \mathrm{~Hz}, 3 \mathrm{H}), 0.91(\mathrm{t}, J=7.3 \mathrm{~Hz}, 3 \mathrm{H}) ;{ }^{13} \mathrm{C} \mathrm{NMR}\left(\mathrm{CDCl}_{3}, 125 \mathrm{MHz}\right)$ $\delta 178.1,83.1,80.4,79.9,78.8,75.9,75.0,70.1,43.6,41.5,36.2,33.8,32.3,26.7,20.8,17.1,15.8,14.5,12.9$, 11.0, 5.5; IR (thin film) 3448, 1718, $1183 \mathrm{~cm}^{-1} ;[\alpha]_{\mathrm{D}}^{23}+9.3(c 0.85, \mathrm{MeOH})\left\{\right.$ lit: $\left.^{7}[\alpha]^{23}{ }_{\mathrm{D}}+9.5(c 2.0, \mathrm{MeOH})\right\}$; HRMS (ESI) $m / z$ calcd for $\mathrm{C}_{21} \mathrm{H}_{40} \mathrm{O}_{8} \mathrm{Na}(\mathrm{M}+\mathrm{Na})^{+}$443.2621, found 443.2625. Anal. Calcd for $\mathrm{C}_{21} \mathrm{H}_{40} \mathrm{O}_{8}: \mathrm{C}_{\text {, }}$ 59.98; H, 9.59. Found: C, 60.02; H, 9.76.

\footnotetext{
${ }^{7}$ Nakata, M.; Arai, M.; Tomooka, K.; Ohsawa, N.; Kinoshita, M. Bull. Chem. Soc. Jpn. 1989, 62, 2618-2635.
} 


\section{Analytical Data}

\section{A. HPLC traces of $( \pm)-13,(+)-13$, and (-)-13:}
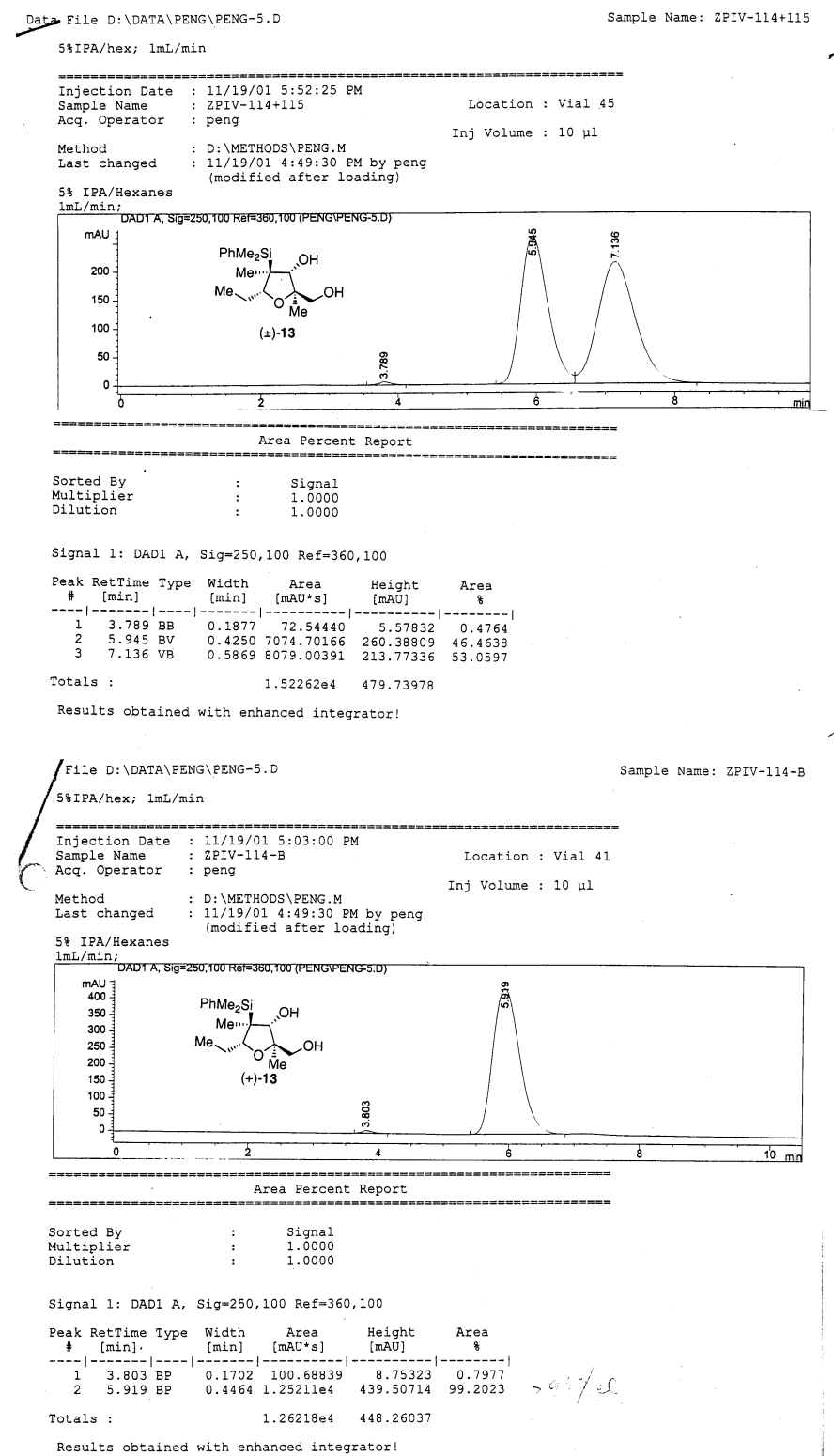

Data File D: \DATA \PENG \PENG-5.D Sample Name: ZPIV-112-C $58 \mathrm{IPA} / \mathrm{hex} ; 1 \mathrm{~mL} / \mathrm{min}$

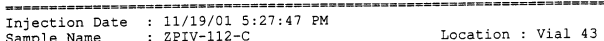
C. Acq. Operator : peng Inj volume : $10 \mu 1$

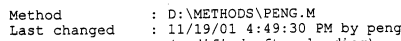
58 (modifled after loading) $58 \mathrm{IPA} / \mathrm{Hexanes}$
$1 \mathrm{~mL} / \mathrm{min} ;$

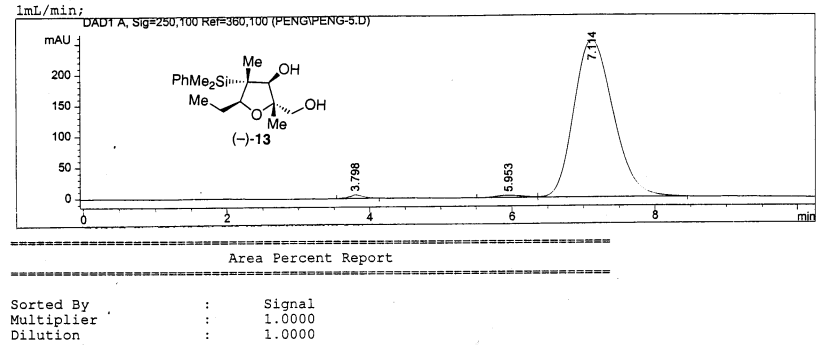

Signal 1: DAD1 A, Sig=250, 100 Ref $=360,100$

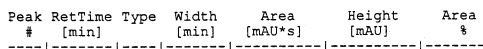

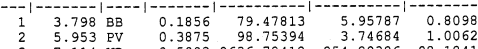

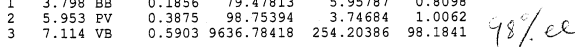
Totals : $9815.01625 \quad 263.90856$ Results obtained with enhanced integrator! 


\section{B: Selected ${ }^{1} \mathrm{H}$ and ${ }^{13} \mathrm{C}$ NMR spectra:}

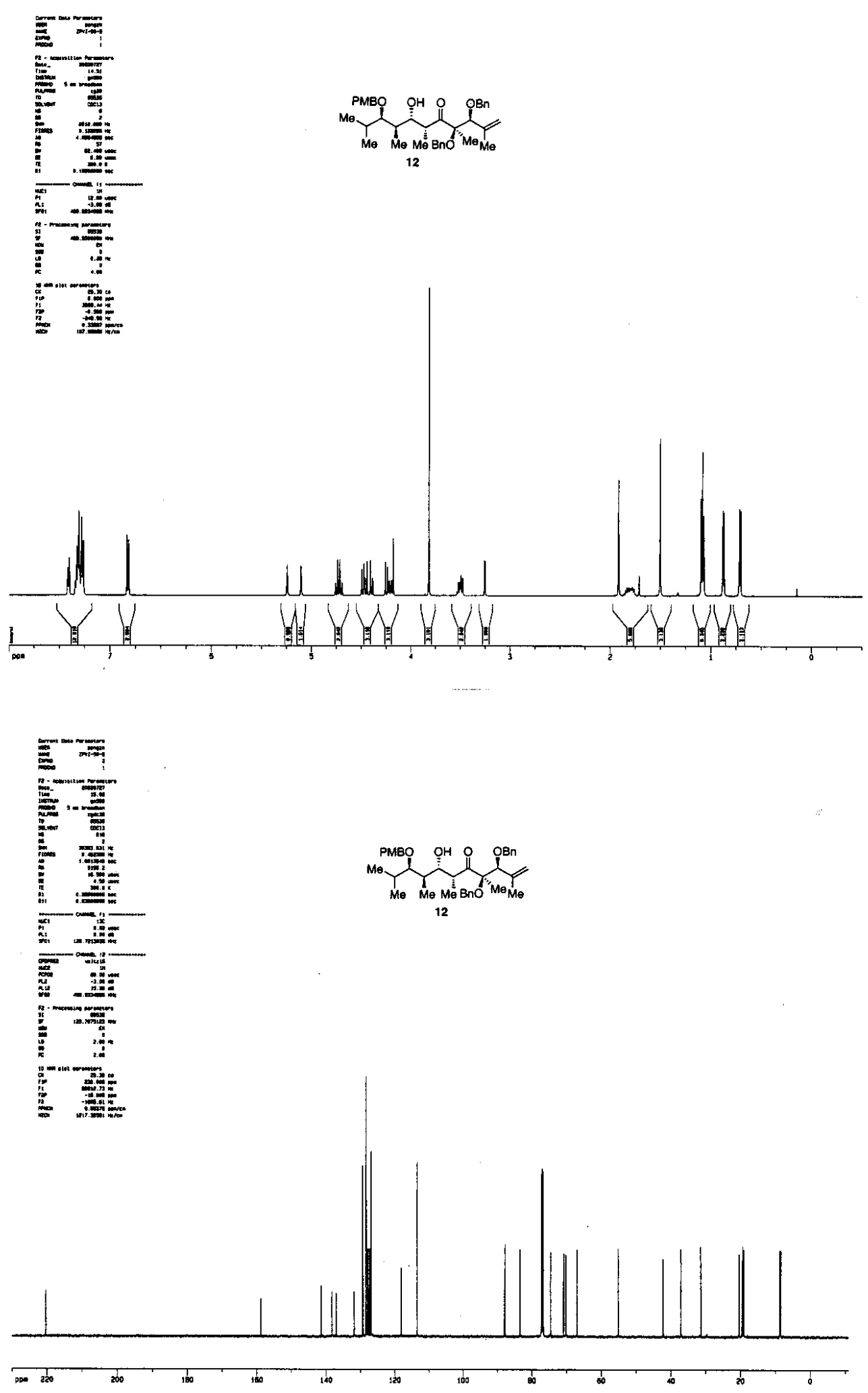



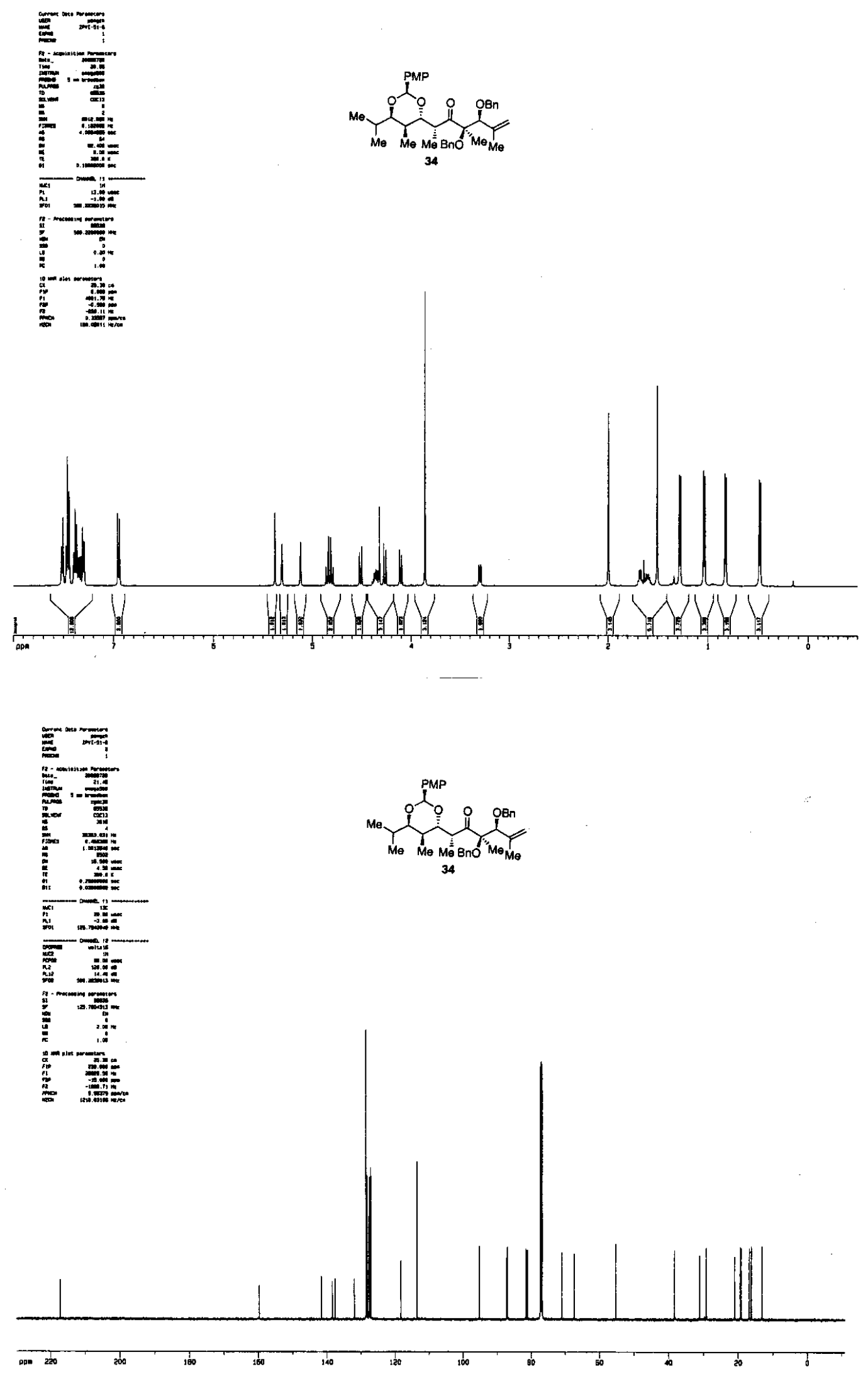

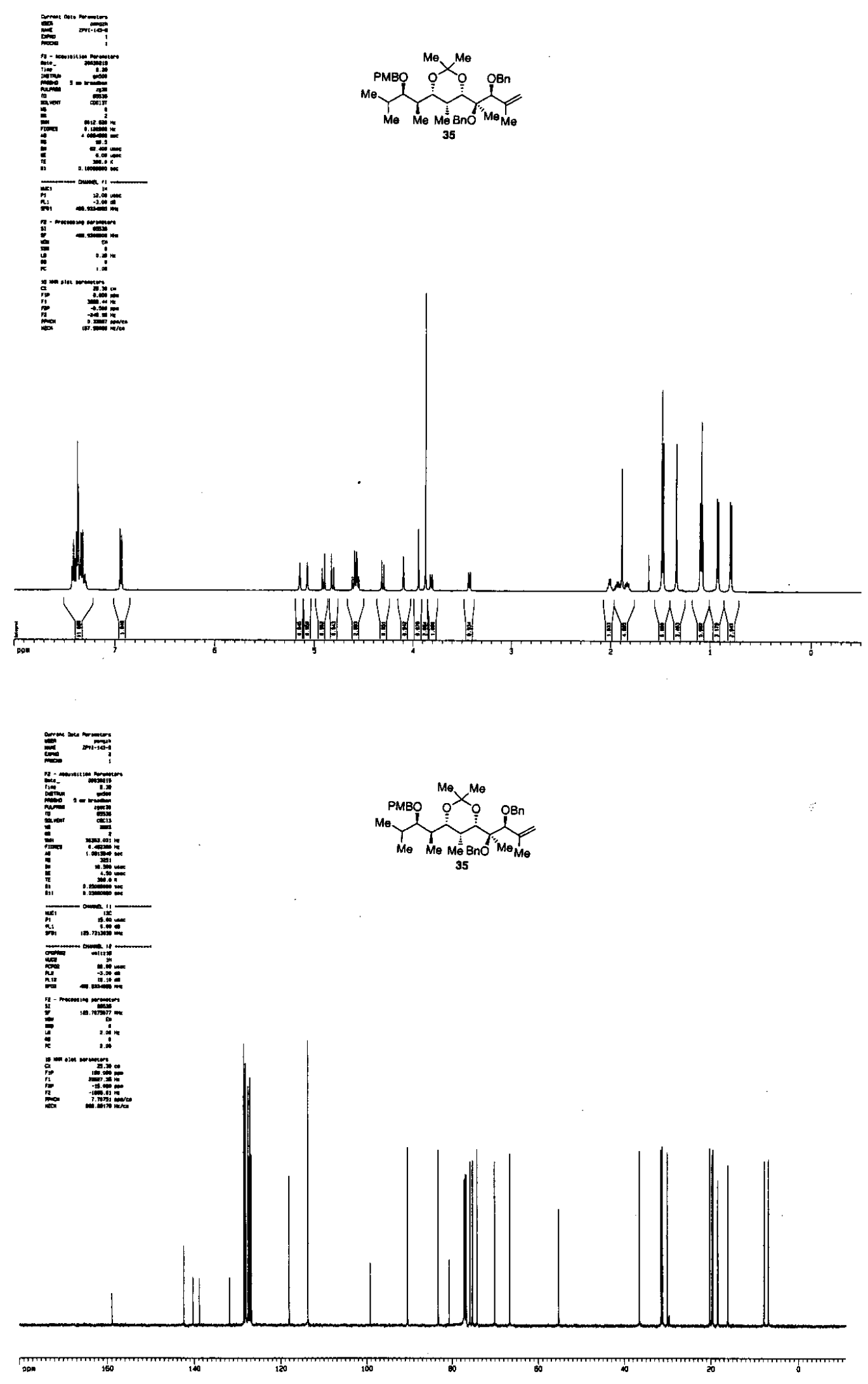

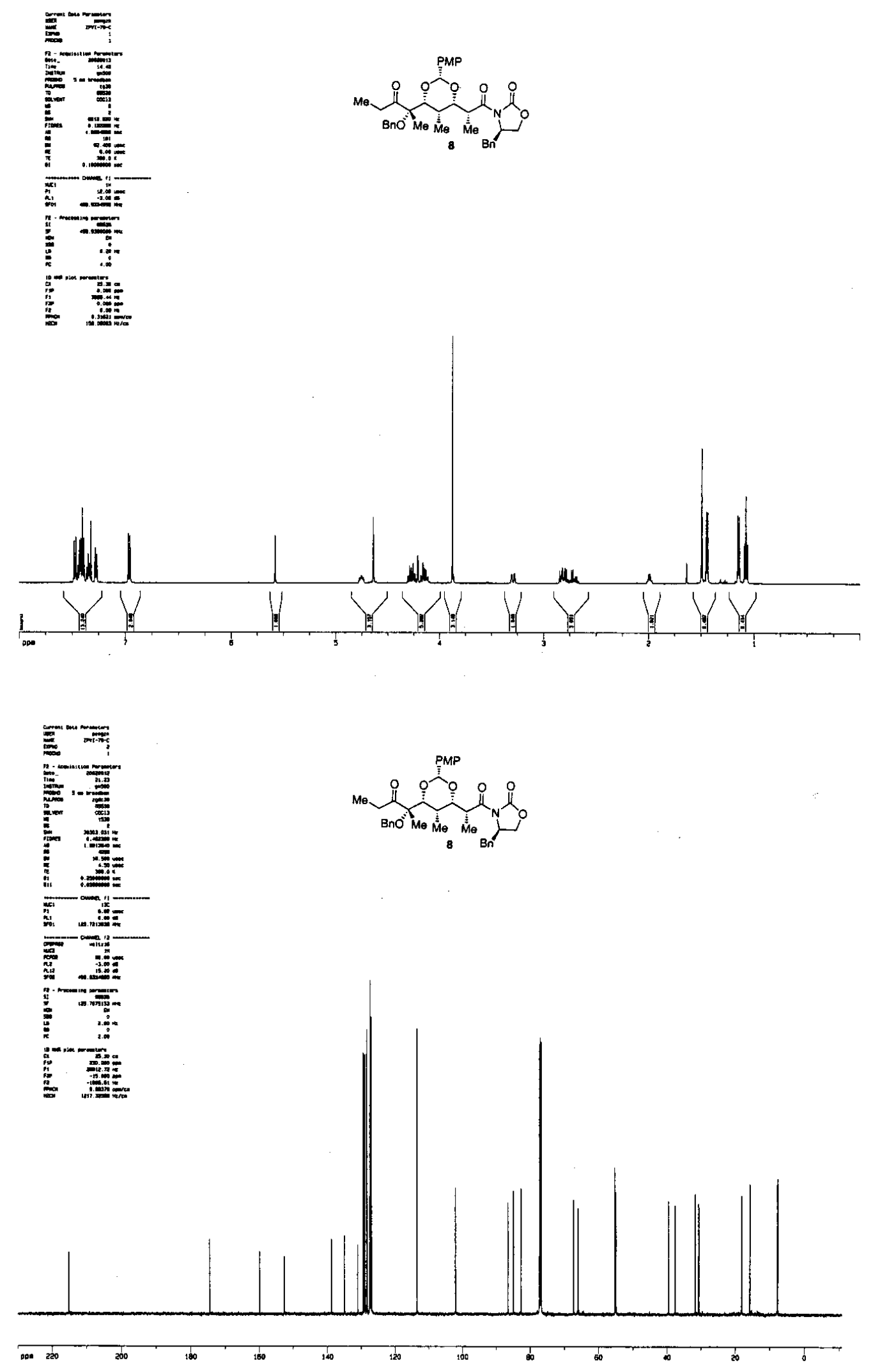

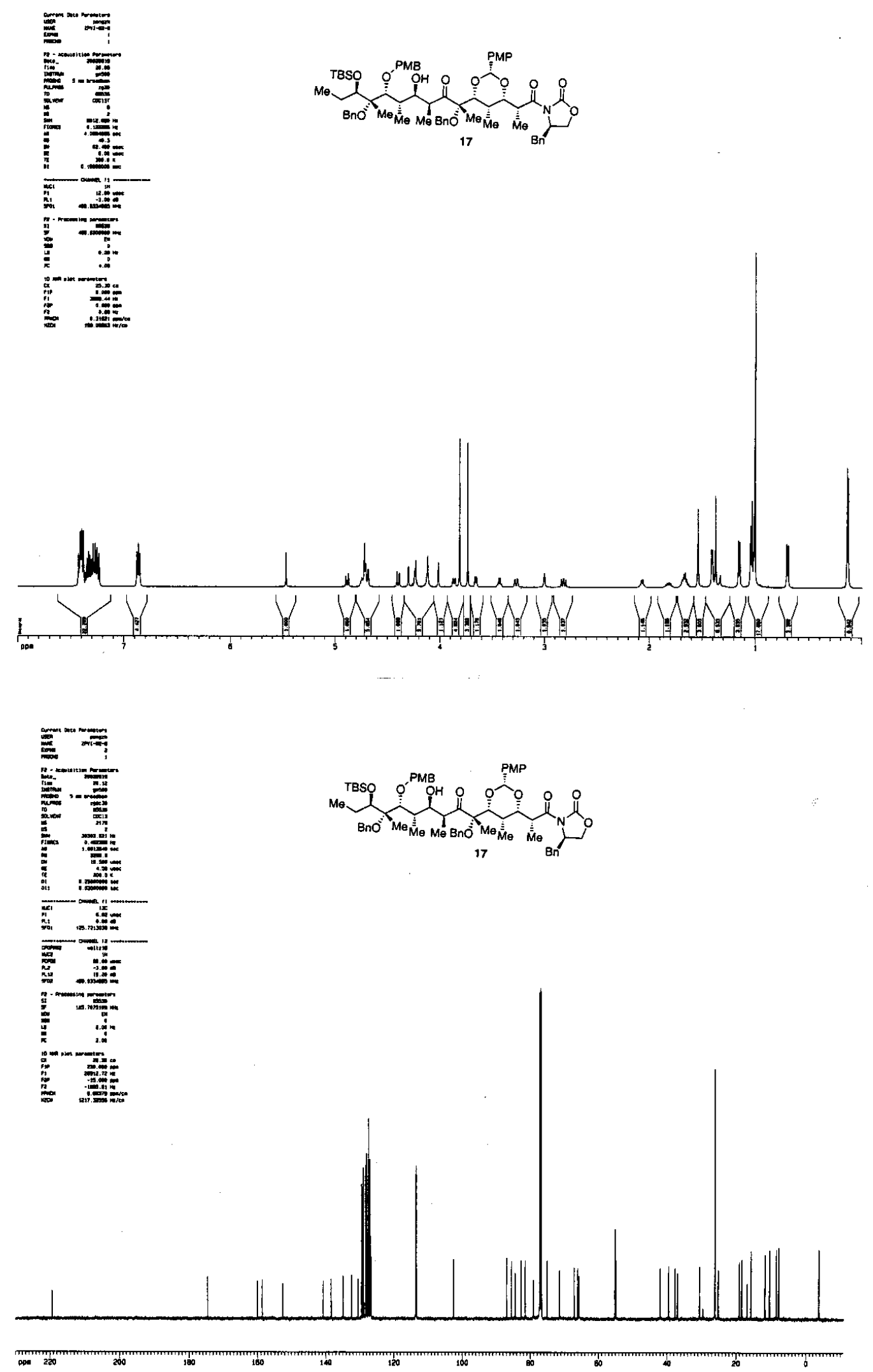

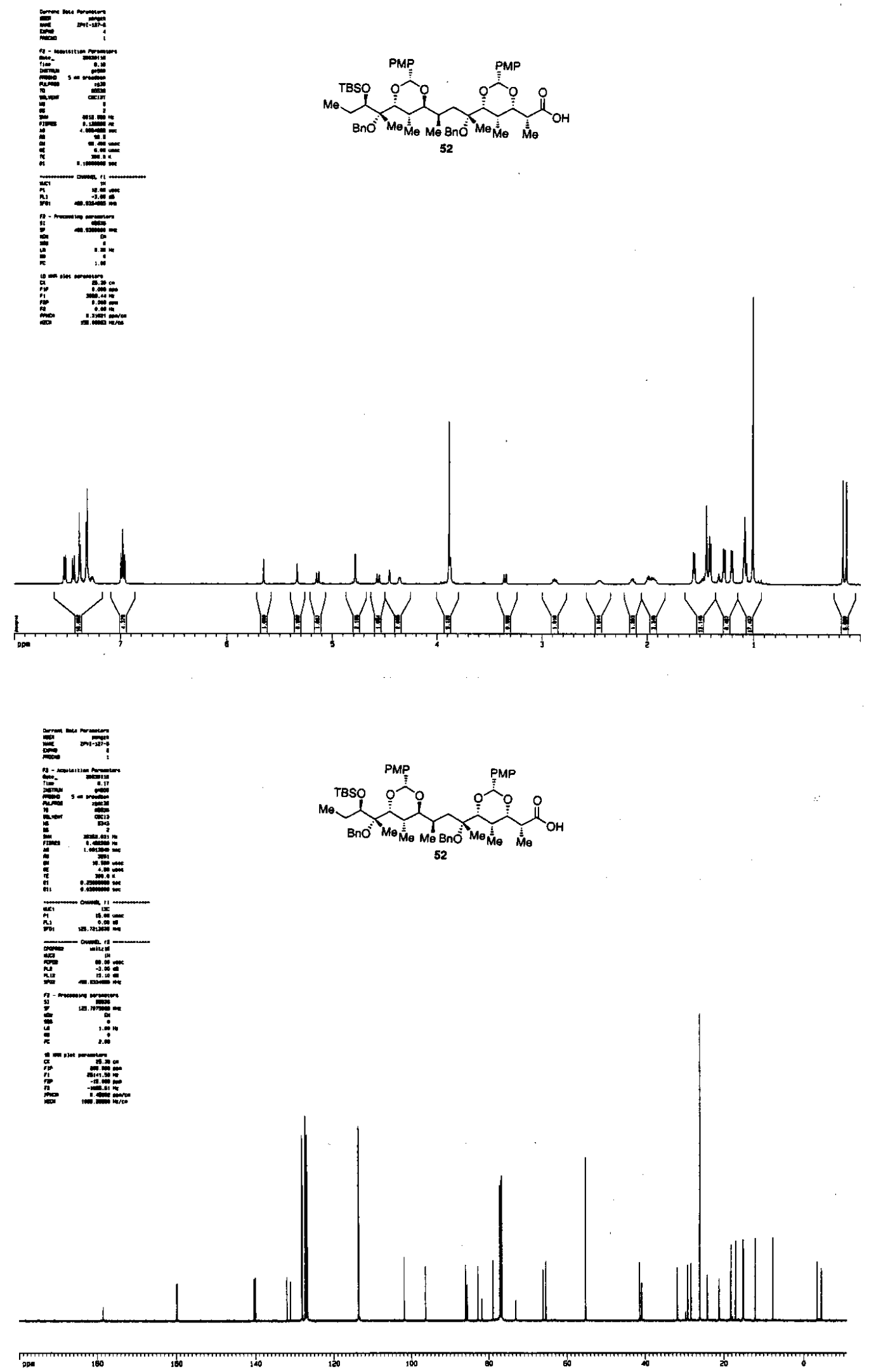
Supporting Information: Peng and Woerpel

S-37
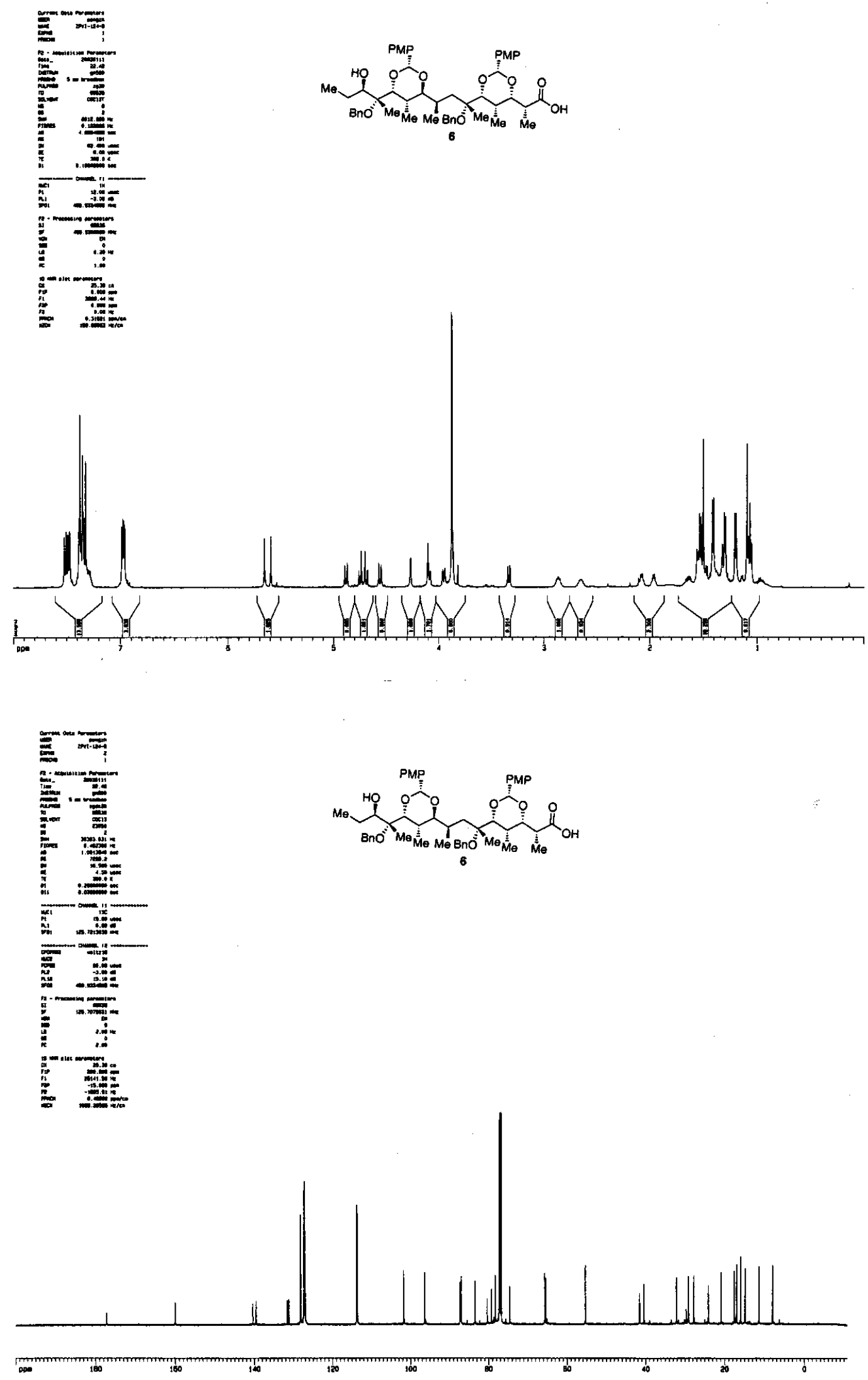

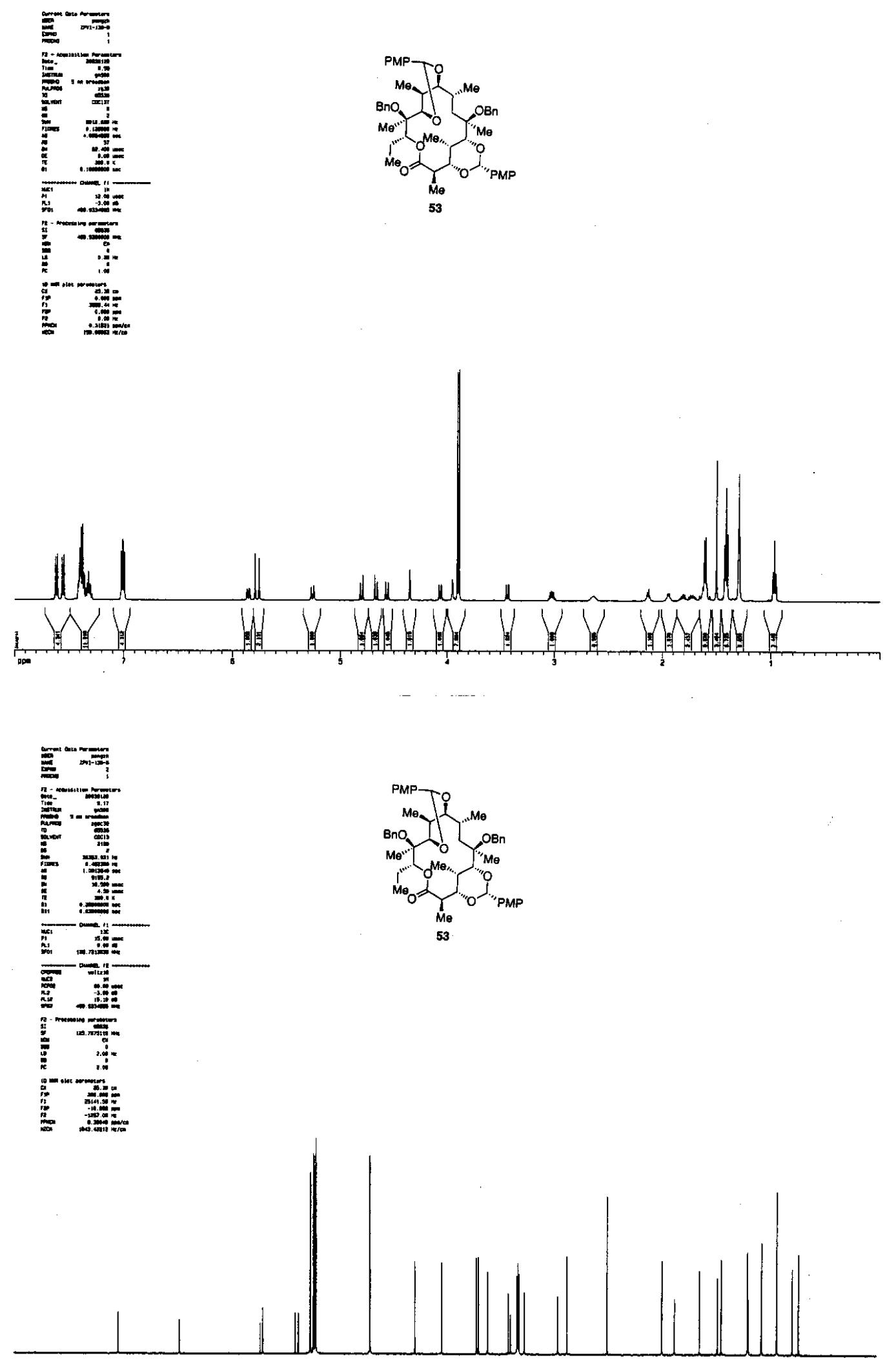

poes

140
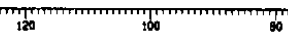

$m_{00}$

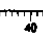



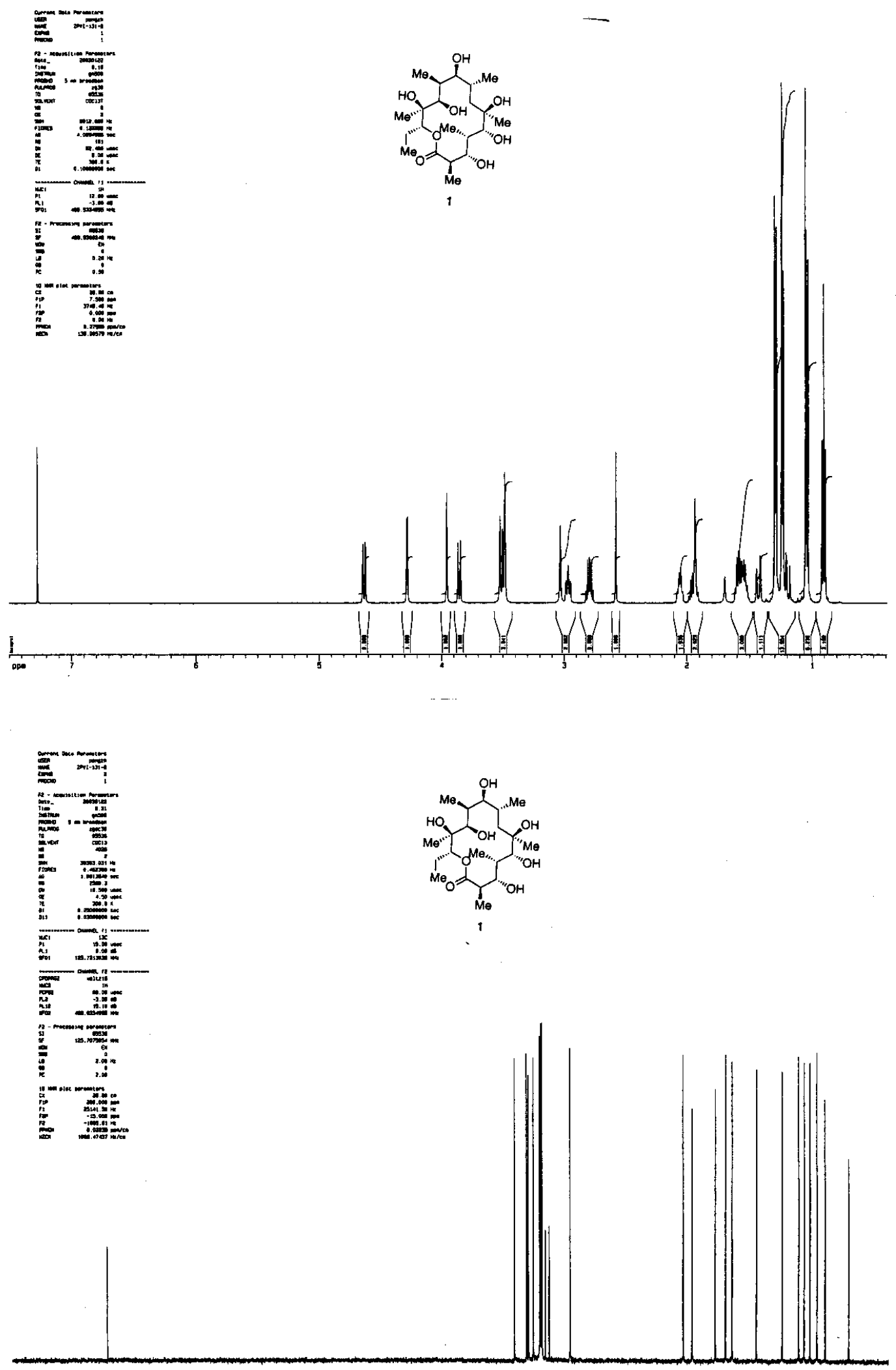

TाT
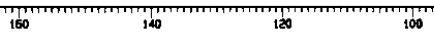

$\lim _{30}$

50

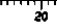


${ }^{1} \mathrm{H}$ NMR spectrum of (9S)-dihydroerythronolide A provided by Professor Kazunobu Toshima:

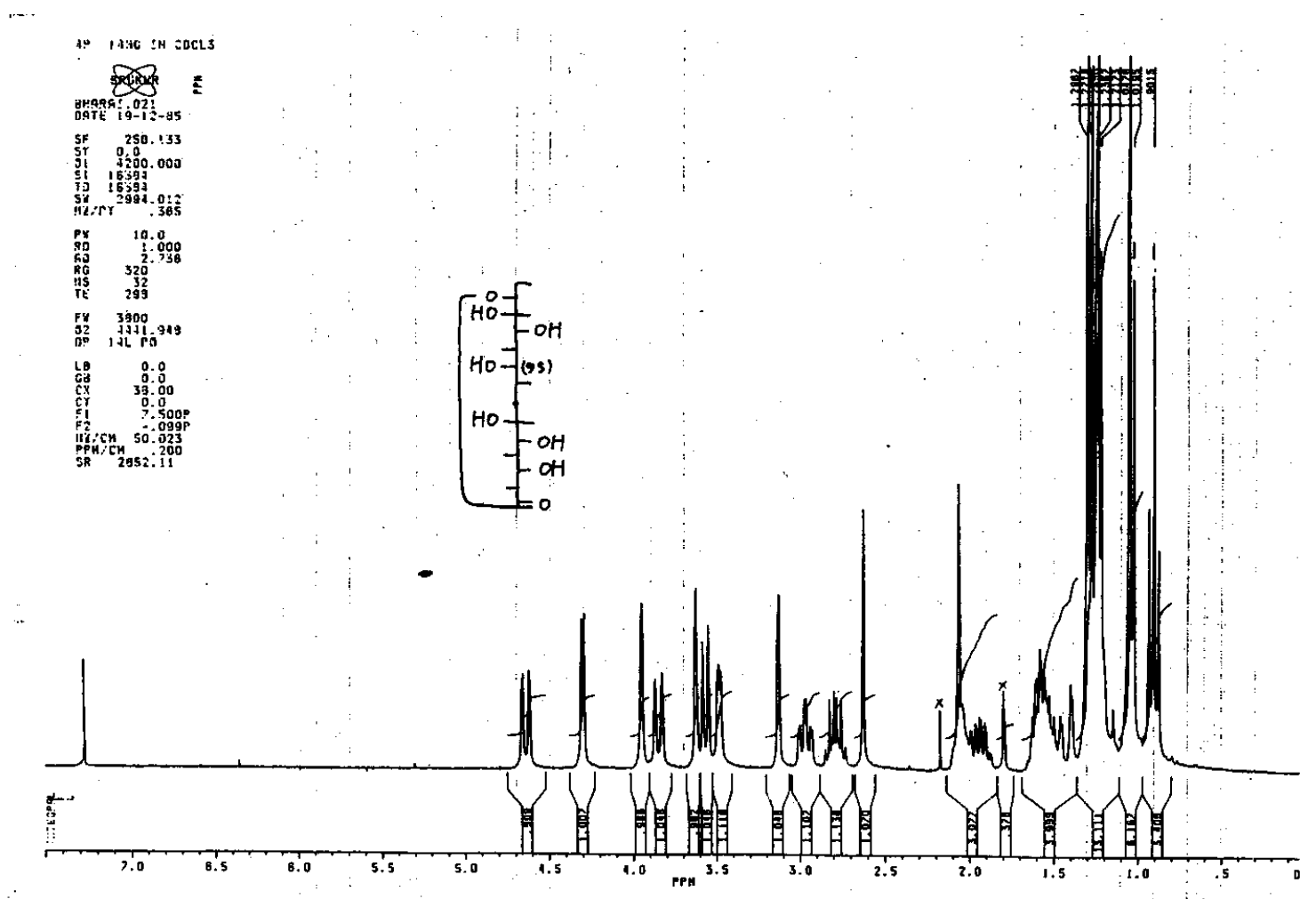

